\title{
An Internet-Based Repository for DEVS Models and their Experimental Frames
}

By

Rachid Chreyh, B.Sc.

A thesis Submitted to

The Faculty of Graduate Studies and Research

In Partial Fulfillment of the requirements of the degree of

Master of Applied Science in Electrical Engineering

\author{
Ottawa-Carleton Institute for Electrical and Computer Engineering \\ Department of Systems and Computer Engineering \\ Carleton University \\ Ottawa, Ontario \\ Canada
}

(C) Copyright 2009, Rachid Chreyh 
Library and

Archives Canada

Published Heritage

Branch

395 Wellington Street

Ottawa ON K1A 0N4

Canada
Bibliothèque et

Archives Canada

Direction du

Patrimoine de l'édition

395 , rue Wellington

Ottawa ON K1A 0N4

Canada

Your file Votre référence

ISBN: 978-0-494-52041-3

Our file Notre référence

ISBN: 978-0-494-52041-3

NOTICE:

The author has granted a nonexclusive license allowing Library and Archives Canada to reproduce, publish, archive, preserve, conserve, communicate to the public by telecommunication or on the Internet, loan, distribute and sell theses worldwide, for commercial or noncommercial purposes, in microform, paper, electronic and/or any other formats.

The author retains copyright ownership and moral rights in this thesis. Neither the thesis nor substantial extracts from it may be printed or otherwise reproduced without the author's permission.
AVIS:

L'auteur a accordé une licence non exclusive permettant à la Bibliothèque et Archives Canada de reproduire, publier, archiver, sauvegarder, conserver, transmettre au public par télécommunication ou par l'Internet, prêter, distribuer et vendre des thèses partout dans le monde, à des fins commerciales ou autres, sur support microforme, papier, électronique et/ou autres formats.

L'auteur conserve la propriété du droit d'auteur et des droits moraux qui protège cette thèse. $\mathrm{Ni}$ la thèse ni des extraits substantiels de celle-ci ne doivent être imprimés ou autrement reproduits sans son autorisation.
In compliance with the Canadian

Privacy A'ct some supporting forms may have been removed from this thesis.

While these forms may be included in the document page count, their removal does not represent any loss of content from the thesis.
Conformément à la loi canadienne sur la protection de la vie privée, quelques formulaires secondaires ont été enlevés de cette thèse.

Bien que ces formulaires aient inclus dans la pagination, il n'y aura aucun contenu manquant.

\section{Canadä}




\section{ABSTRACT}

The development of simulation models for complex systems can be difficult and timeconsuming; therefore, model reuse is of high value to model designers. The availability of context-of-use information and reusable experiments for modelling components is important for promoting their reuse. This work presents an architecture for an internetbased repository of DEVS models and their Experimental Frames. The proposed architecture uses Experimental Frames to capture the context for the models and allow the sharing of experiments. The proposed architecture specifies the details of the required storage entities, including their contents and relationships. It simplifies the tasks of searching and contributing to the repository. It also allows for an 'open source', internetbased repository of DEVS models and experiments to be built. Finally, the architecture is interoperable for different DEVS tools and thus presents a small step towards enabling collaboration between these tools. Based on this architecture, a prototype repository was implemented. 


\section{ACKNOWLEDGEMENTS}

First of all, I thank God the almighty for giving me guidance and the ability and patience to complete this research.

I would like to gratefully acknowledge the support of my supervisor, Dr. Gabriel Wainer, who guided my research and helped shape it. His constant support, kindness, and patience were very important in allowing the successful completion of this work.

I would also like to thank my parents, grandmother, and siblings for giving me the strength to complete this work. This work would not have been possible without their constant encouragement and support.

Finally, I would like to thank my wife, Reem, for her constant support and encouragement during my work on this research. Her understanding and endless patience throughout the course of my research were instrumental in allowing me to successfully complete this work. I dedicate this work to her. 


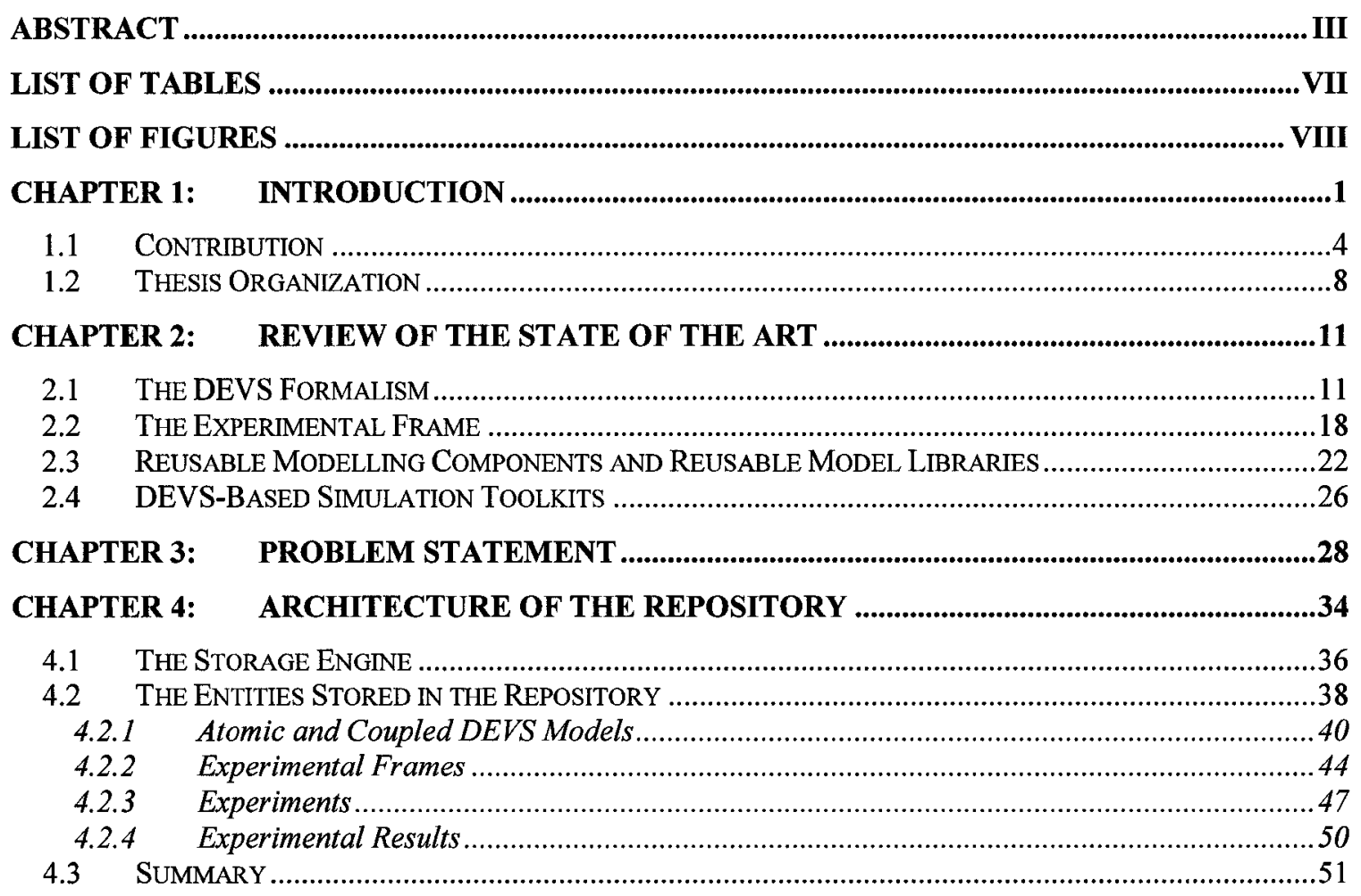

CHAPTER 5: THE CD++ REPOSITORY - SOFTWARE ARCHITECTURE.........................53

5.1 OVERVIEW OF THE CD++ REPOSITORY SOFTWARE ARCHITECTURE ........................................53

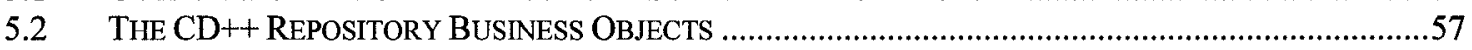

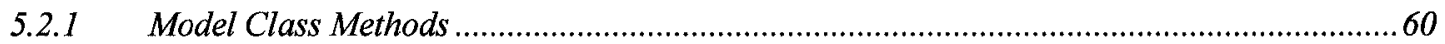

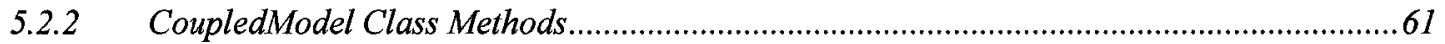

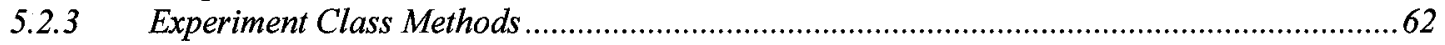

5.2.4 ExperimentalResults Class Methods..........................................................................63

5.2.5 Methods for all Business Object Classes..................................................................63

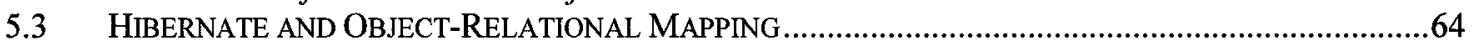

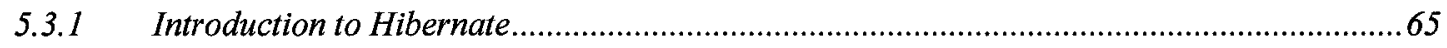

5.3.2 Mapping CD++ Repository Business Objects to Database Tables................................6.69

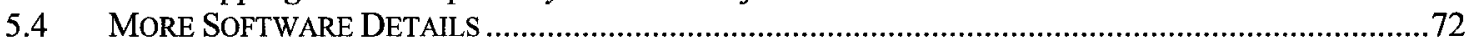

CHAPTER 6: CD ++ REPOSITORY -THE CLIENT APPLICATION...................................74

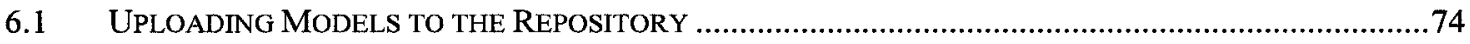

6.1.1 The Model Definition (.ma) File Structure ...............................................................75

6.1.2 Automatic Detection of Model Kind, Structure, Name and Conflicts...................................77

Collection of User Entered Data ................................................................................81

6.2 UPLOADING EXPERIMENTAL FRAMES, EXPERIMENTS AND EXPERIMENTAL RESULTS...................83

6.3 SEARCHING AND DOWNLOADING MODELS AND THEIR EXPERIMENTS .....................................88

6.3.1 Searching for and Downloading Models......................................................................8.

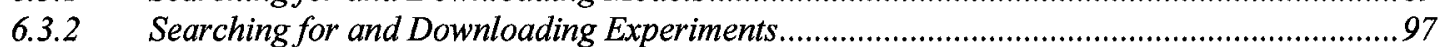

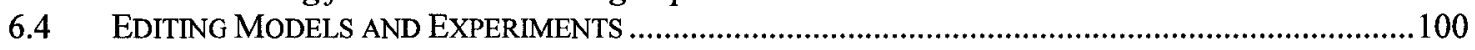


CHAPTER 7: TESTING THE CD++ REPOSITORY ….............................................................103

CHAPTER 8: CONCLUSION AND FUTURE WORK …...........................................................110

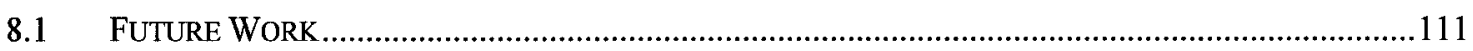

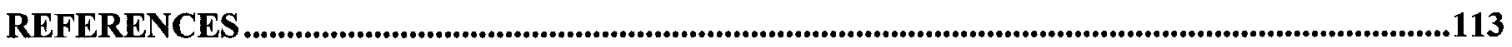

APPENDIX-A：CD++ REPOSITORY SOFTWARE DETAILS ...........................................................118 
List of Tables

Table 1: Comparison with Exiting Model Repositories

Table 2: Table of Model Zip Files Used During Testing 104

Table 3: Tests and Results for Model-Based Operations 106

Table 4: Tests and Results for Experiment-Based Operations. 107 
List of Figures

Figure 1: Definition of an Atomic DEVS Model [2]....................................... 13

Figure 2: Structure of a Coupled DEVS Model ............................................ 15

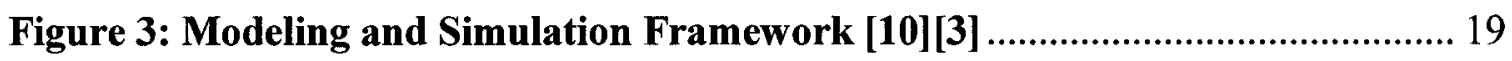

Figure 4: Experimental Frame System Specification [3][10]............................. 20

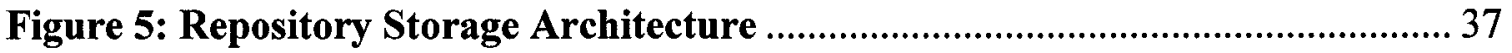

Figure 6: Structure of a Stored DEVS Coupled Model........................................ 44

Figure 7: Structure of a Stored DEVS Coupled Model......................................... 47

Figure 8: CD++ Repository Software Architecture....................................... 56

Figure 9: CD++ Repository Business Objects Class Diagram ................................. 59

Figure 10: A High Level View of the Hibernate Architecture ................................ 66

Figure 11: Sample Hibernate Mapping File ................................................... 71

Figure 12: CD++ Repository Relational Database Tables. ................................... 72

Figure 13: Sample Atomic and Coupled (.ma) Files....................................... 77

Figure 14: Atomic Model Upload Window Screenshot - Part1 .......................... 82

Figure 15: Atomic Model Upload Window Screenshot - Part2 ............................ 83

Figure 16: Event Based Experiment Upload Window Screenshot..........................86

Figure 17: Model Based Experiment Upload Window Screenshot ......................... 87

viii 
Figure 18: Experimental Results Upload Window Screenshot

Figure 19: Model Search Window Screenshot

Figure 20: Search Results Window Screenshot

Figure 21: Download Window Screenshot 96

Figure 22: Experiment Search Window Screenshot. 98

Figure 23: Experiment Download Window Screenshot 100

Figure 24: Concurrent editing of Models Test 108

Figure 25: Concurrent Upload of Models with Same Name Test 109

Figure 26: The gui.upload Java Package Structure 126

Figure 27: The gui.download java Package Structure 131 


\section{Chapter 1: Introduction}

In the field of software engineering, the concept of creating reusable software components is of great importance to the reliability, maintainability and to reducing the development costs of the software [1]. For instance, object-oriented methodologies and programming languages have been developed in part to support this concept of reusability. Using these methodologies and languages, libraries of reusable components can be created and documented so that software application developers can use them in the future. For example, one can find a vast amount of Java packages that can be re-used by Java programmers to build new applications. Having reusable components means that a given component is designed, developed and tested only once; it is then kept with other components in a special library such that developers can reuse it in the future. Software development teams who make use of reusable components in their applications will benefit from the reduction in development cost (because the reusable components are already developed). They will also benefit from the fact that the reusable components are most likely very reliable (since any bugs would most probably have been fixed during the first application's testing cycle). Finally, the software will be easier to maintain because at least some developers are likely to be familiar with the some of the reusable components if they have used them before.

In the field of computer based Modeling and Simulation (M\&S), the same benefits exist through the creation of reusable simulation models. The Discrete Event System 
Specification (DEVS) [2] can provide these benefits. DEVS is a formalism for the modeling and simulation of discrete event systems that provides a framework for the creation of hierarchical and modular simulation models. This hierarchical and modular approach to model creation allows models to be independently tested and reused thus enhancing reliability, maintainability and reducing the effort required for model development and testing. In addition to the hierarchical and modular nature of DEVS models, the DEVS architecture separates the model from the simulator, thus allowing the execution of a given DEVS model on different simulator implementations [2].

Creating a DEVS model involves examining the system to be modeled (the source system) through an "Experimental Frame". An Experimental Frame (EF) is a specification of the conditions under which a system is observed or experimented with [2]. The Experimental Frame defines the data being collected and the conditions under which the source system is being observed; in other words, it defines the context within which the source system is being observed. A DEVS model thus aims to approximate the behaviour of the source system within the parameters set by the Experimental Frame. When a model is built, a DEVS simulator can be used to execute the model to produce its intended outputs. The validity of a DEVS simulation model is closely related to the parameters set by the experimental frame; in other words, a model of a source system is only valid in the context for which the model was built. 
The main motivation for this work is the desire to share DEVS models between different users and the great benefits that this brings to the modelling process. As will be discussed in later chapters, there are currently many DEVS tools in existence, but none provide a means for sharing the DEVS models developed by the users of these tools. As will also be discussed in later chapters, the model libraries in existence today are not suitable for sharing DEVS models in a way that makes them publicly available. This ability to share DEVS models necessarily promotes DEVS model reuse. Reuse of DEVS modelling components greatly reduces the time and effort required when modelling complex systems. This is because the availability of existing modular DEVS components that can be "connected" together into more and more complex models means that, at least in part, the complex system can be modelled by connecting the appropriate reusable DEVS components together, thus reducing the effort and time required to model the system. A second closely related motivation for this work is the desire to share the context of use information about the shared DEVS models. Since the model of any real system is only valid within the context for which the model was built [3], sharing this context information is essential in order to be able to properly reuse DEVS models. As will be shown in later chapters, many of the existing model libraries do not provide the context of use information for the stored models. A third related motivation is the desire to also share the experiments that were used to verify the correctness of the DEVS models. These experiments can be difficult and time consuming to develop, and being able to share them makes re-using the models that much easier; in addition, the availability of 
these experiments provides confidence in the correctness of the models for which they were built. As will be shown in later chapters, none of the existing model libraries includes the concept of sharing the model's experiments. The final motivating factor for this work is that the increasing complexity of the systems that are being modeled and the subsequent increase in the geographical separation between model design teams working with the same design data has created a need to share this design data over large distances. As a result, there is a desire to be able to share all the previously mentioned items between modellers located at different geographical locations. Consequently, the goal of this work is to develop an architecture for a system that firstly links together the DEVS models, the context of use for those models, and the experiments used to verify the models; and secondly enables the sharing of a DEVS model, its context information, and its experiments over the internet.

\subsection{Contribution}

The inability to share DEVS models, their conceptual information (including the context of use), and their experiments presents obstacles to the ability of modellers to apply the desirable concept of model reuse. This work tries to provide a solution to this problem by developing an architecture for a DEVS model library that can hold all of this information about the DEVS models and is accessible over the internet. As will be shown in later chapters, our research yielded no systems or architectures in existence today that provide 
such information about models and that can be publicly available. Although there are many DEVS simulation tools in existence today, none of them provide a means for sharing DEVS models between users. In addition, many of the model libraries in existence are either specialized for modelling certain systems, or concentrate on creating modular components to facilitate model re-use (which is something that automatically exists for DEVS models), or they do not hold all the required context of use and experiment information. Importantly, none of the mode libraries in existence today is capable of storing, along with the models, a set of experiments that can be run for them.

An important contribution of this work is the idea of storing the Experimental Frame along with the DEVS models. The EF holds information about the context of use for the model to which it belongs, as well as containing links to the experiments that can be run on the model. This enables the sharing of the models and their experiments among modellers. In addition, as will be described in later chapters, the proposed architecture supports the reuse of the experiments by different DEVS models, meaning that a given experiment may equally be applicable to more than one DEVS model, and thus be 'linked' to all of them.

A second contribution of this work is that it specifies the information that needs to be stored for each entity kept in the repository. The main storage entities discussed in this work are the Models, Experimental Frames, Experiments, and Experimental Results. 
From a high-level view, one can easily talk about storing these entities in a repository; a closer look, however, reveals that there are details regarding what exact information needs to be stored for each entity. Each stored entity requires its own specific descriptive information and may require the storage of certain files for example. In addition, the relationships between these entities need to be represented in a manner that allows storing them in the repository. This work provides a solution to these problems.

A third contribution of this work is the creation of an architecture that supports an "Open Source" repository of DEVS models and experiments. Our research shows that at this time there are no such "Open Source" model repositories in existence. The 'open source' concept means that modellers can use an internet connection to upload and download DEVS modeling components and experiments to/from this repository. A user looking for DEVS models of ATM machines, for example, can search this repository and download what they need. Similarly, any user can add models and/or experiments to this repository with no need for a 'librarian' to interfere.

Another contribution of this work is that it provides a small step in the direction of allowing collaboration among users of different DEVS tools. While this work is not directly concerned with solving the problem of interoperability between different DEVS simulation tools, providing a repository that enables the sharing of DEVS models and experiments is a small step that is required to enable users with different tools to share 
their models once the other obstacles to interoperability are solved.

Finally, a prototype application, the CD++ Repository, was built based on the proposed architecture to prove its effectiveness. There are many different DEVS Modeling and Simulation platforms in existence today. In this work, the CD++ Builder platform is the one for which the prototype repository application is developed. The $\mathrm{CD}++$ Builder toolkit [4] is built as a plug-in for the Eclipse IDE. It enables the users to develop DEVS and Cell-DEVS models and run their simulations in a convenient and practical environment. The CD++ Repository application was built to work with and be part of the $\mathrm{CD}++$ Builder toolkit. The CD++ Repository is composed of two main parts: an Internet based database server and a client application. The database contains the stored entities (Models, EF, Experiments, and Experimental Results) and their relationships. The client application is embedded within the $\mathrm{CD}++$ Builder toolkit in Eclipse and is used by a modeller to either upload, or search for and download the desired DEVS models and EF or the desired experiments or experimental results.

Besides the theoretical contributions related to the design of an architecture for a repository of DEVS models and their EF, this work contributes a couple of tangible software deliverables that can benefit interested researchers. First, as mentioned above, a prototype repository, the $\mathrm{CD}++$ Repository, was created as part of this work. As a result, there is currently a repository of DEVS models and EF that can be utilised by CD++ Builder users from anywhere in the world. Second, as will be explained in a later chapter, 
the $\mathrm{CD}++$ repository has a layered software architecture composed of a presentation layer and a persistence layer. While the presentation layer's software is specific to the CD++ Builder Toolkit, the persistence layer's software is general in nature. Therefore, other researchers interested in building a repository for their DEVS tools following the architecture presented in this work can use this piece of software as the interface to their database. In addition, the design of the persistence layer allows, with minor changes, interested researches to host it on a web-server and use web-services to expose its main "services" thus allowing the creation of web-based client applications for the repository.

On a final note, a paper [5] based on the work in this thesis has been written and presented in the SpringSim'09 conference in San Diego California. In addition, a paper [6] on Cell-DEVS models for fire spreading analysis has been written and presented in the seventh international conference on cellular automata for research and industry in Perpignan, France.

\section{$1.2 \quad$ Thesis Organization}

The rest of this Thesis is organized as follows:

Chapter 2 introduces the Discrete Event System Specification (DEVS) and the CellDEVS formalisms. A formalism for defining Experimental Frameworks is also 
introduced. Then several works discussing reusable modelling components and model libraries are presented. Finally, a brief survey of existing DEVS simulation toolkits is presented.

Chapter 3 presents the problems that this thesis is concerned with solving. It then goes on to describe how the existing model libraries introduced in the previous chapter are not suited to solving the problem at hand. Finally, it presents a brief description of how these problems are solved by the proposed repository's design.

Chapter 4 describes the proposed architecture for the repository. The storage engine is described, then the storage entities that are used to store the information are described in detail. This chapter also illustrates how the relationships between these entities are stored.

Chapter 5 describes the software architecture of the CD++ Repository application, a prototype application that implements the proposed architecture. It starts with an overview of the architecture, and then proceeds to describe the business objects and their mapping to database tables.

Chapter 6 describes the features and capabilities of the CD++ Repository's client application in terms of uploading, searching, downloading, and editing the stored models and experiments. 
Chapter 7 describes the testing that was performed on the CD++ Repository by using it to store, retrieve and edit a number of CD++ DEVS models selected from a collection of existing models.

Chapter 8 presents the main conclusions of the thesis, and present possible future work. 


\section{Chapter 2: Review of the State of the Art}

This chapter reviews the state-of-the-art in the modelling and simulation field, specifically in aspects that are useful in building libraries of reusable DEVS components. The original DEVS formalism is first introduced followed by the Cell-DEVS formalism. Then the Experimental Frame concept is discussed as a way to capture the context within which a model is designed; then a formalism of the Experimental Frame is given. Then, the hierarchical models library (HMLib [7]) is introduced as an example of an existing models library. Finally, a brief survey of existing DEVS simulation toolkits is given with emphasis on the CD++ Builder Toolkit, and the lack of a model's database feature in other toolkits.

\subsection{The DEVS Formalism}

The Discrete Event System Specification (DEVS) formalism [2] provides a sound and formal M\&S framework. It also supports the hierarchical and modular development of models. Using DEVS a system can be described using atomic and coupled DEVS models. The atomic models are the basic building block of any DEVS model. They define the behavior of a component in the system. The coupled models are structural in nature in that they are composed of an interconnection of other atomic and/or coupled models.

The following is the formal definition of an Atomic model [2]: 


$$
\mathrm{M}=<\mathrm{X}, \mathrm{S}, \mathrm{Y}, \delta_{\text {int }}, \delta_{\text {ext }}, \lambda, \text { ta }>
$$

Where

$\mathrm{X}$ is the set of external input events;

$\mathrm{S}$ is the set of sequential states;

$\mathrm{Y}$ is the set of outputs;

$\delta_{\text {int: }} \mathrm{S} \rightarrow \mathrm{S}$ is the internal transition function;

$\delta_{\text {ext }}: \mathrm{Q} \times \mathrm{X} \rightarrow \mathrm{S}$ is the external transition function, where

$\mathrm{Q}=\{(\mathrm{s}, \mathrm{e}) \mid \mathrm{s} \varepsilon \mathrm{S}, 0 \leq \mathrm{e} \leq \mathrm{ta}(\mathrm{s})\}$ is the total state set where $\mathrm{e}$ is the elapsed time since the last transition.

$\lambda: \mathrm{S} \rightarrow \mathrm{Y}$ is the output function;

ta: $\mathrm{S} \rightarrow \mathrm{R}_{0 \rightarrow \infty}$ is the time advance function;

The behaviour of an Atomic DEVS model can be described as follows. A model starts at a given state, $s$, and will remain in that state for an amount of time defined by the time advance function ta(s). If during this time an external event is received at one of the input ports, the external transition function is executed to determine the new state of the model. If no external events are received and the time defined by ta(s) elapses then the output function $\lambda$ is executed the resulting output value is presented at the output port, then the internal transition function is executed to determine the next state of the model. This 
behaviour can be visualized by the diagram in Figure 1 [2] :

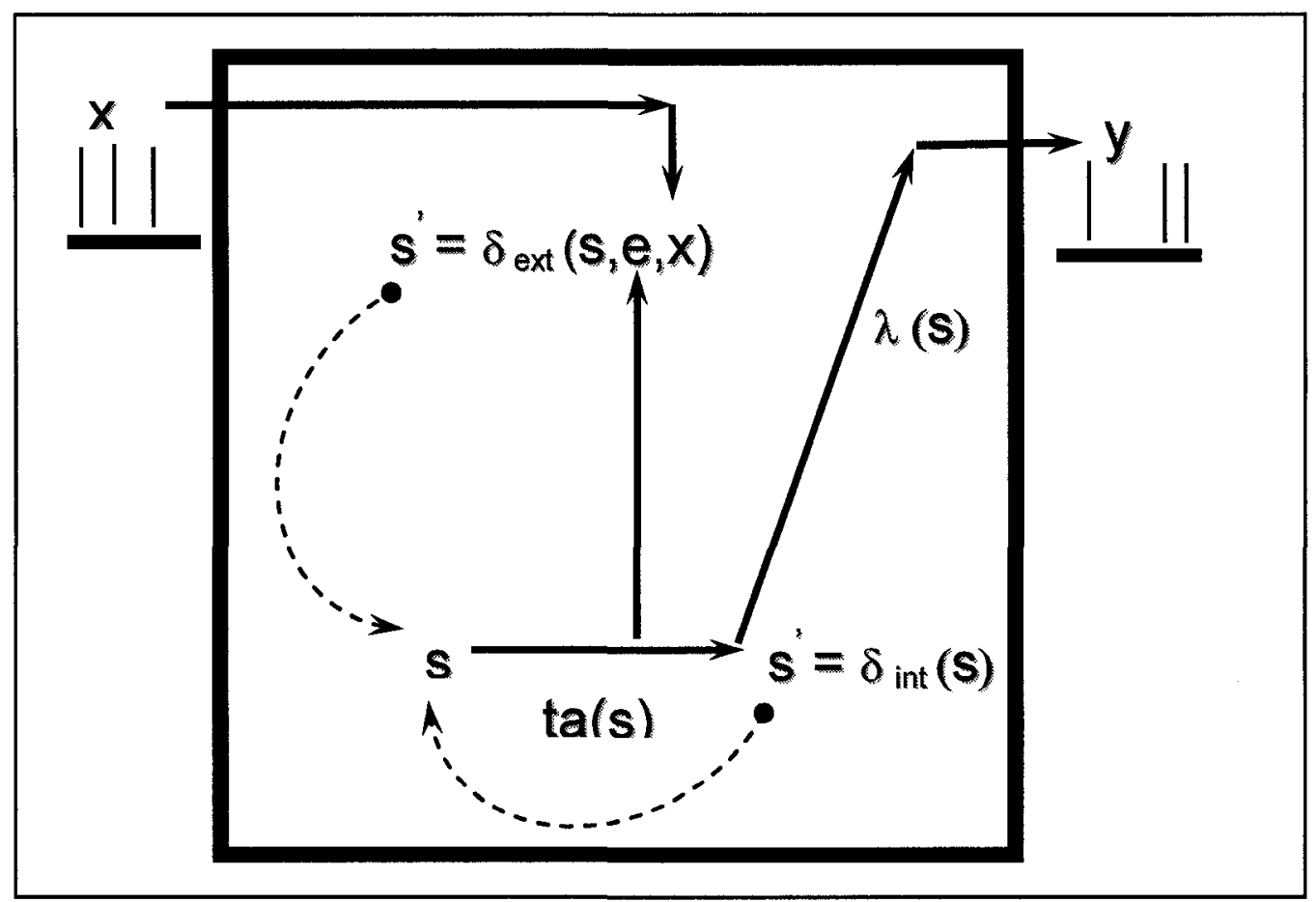

Figure 1: Definition of an Atomic DEVS Model [2]

The formal definition of the DEVS coupled model on the other hand is [2]:

$$
\mathrm{CM}=<\mathrm{X}, \mathrm{Y}, \mathrm{D},\left\{\mathrm{M}_{\mathrm{i}}\right\},\left\{\mathrm{I}_{\mathrm{i}}\right\},\left\{\mathrm{Z}_{\mathrm{ij}}\right\}, \varsigma>
$$

Where

$\mathrm{X}$ is the set of input events;

$\mathrm{Y}$ is the set of output events;

$\mathrm{D}$ is an index for the components of the coupled model;

$\forall \mathrm{i} \& \mathrm{D}, \mathrm{M}_{\mathrm{i}}$ is a basic DEVS model, defined by 
$\mathrm{M}_{\mathrm{c}}=<\mathrm{I}_{\mathrm{i}}, \mathrm{X}_{\mathrm{i}}, \mathrm{S}_{\mathrm{i}}, \mathrm{Y}_{\mathrm{i}}, \delta_{\text {inti }}, \delta_{\text {exti }}$, ta $>$

$\left\{I_{i}\right\}$ is the set of influencees of model $\mathbf{i}$ (that is, the models that can be influenced by outputs of model i);

$\left\{Z_{\mathrm{ij}}\right\}$ is a set where $\forall \mathbf{j} \in \mathrm{I}_{\mathrm{i}}, Z_{\mathrm{ij}}$ is the $\mathrm{i}$ to $\mathrm{j}$ translation function;

$\varsigma$ is the tie-breaking selector;

To elaborate on the above definition, a coupled model is composed of a number of basic models " $\mathrm{M}_{\mathrm{i}}$ ". Each model's outputs are connected to the inputs of its influencee models $\left\{I_{\mathrm{i}}\right\}$. The $Z_{\mathrm{ij}}$ function translates the outputs from component $i$ to the inputs of component $j$, and thus defines the coupling of the inputs and outputs of the components. The tiebreaking selector is needed because of the possibility that the internal transition function of more than one component can occur at the same time. It defines an order over the components so that only one will have its internal transition function trigger at any time. Finally it is important to note the concept of closure under coupling, which basically means that for every coupled DEVS model there is an equivalent atomic DEVS model. This implies that the basic components that make up a given coupled model can themselves be atomic or coupled models.

Figure 2 below illustrates the structure of a sample coupled DEVS model. As can be seen the top model, Coupled Model \#1 is composed of two child coupled models (Coupled 
Model \#2 and Coupled Model \#3) and an atomic model (Atomic Model \#6). The inputs of the top model are coupled to the inputs of the two child coupled models, the outputs of the child coupled models are coupled to the inputs of Atomic Model \#6, and finally the output from Atomic Model \#6 is coupled to the output of the top model. Similarly, the child coupled models are composed of child atomic models and the inputs and outputs of the coupled models and their children are coupled as illustrated in Figure 2.

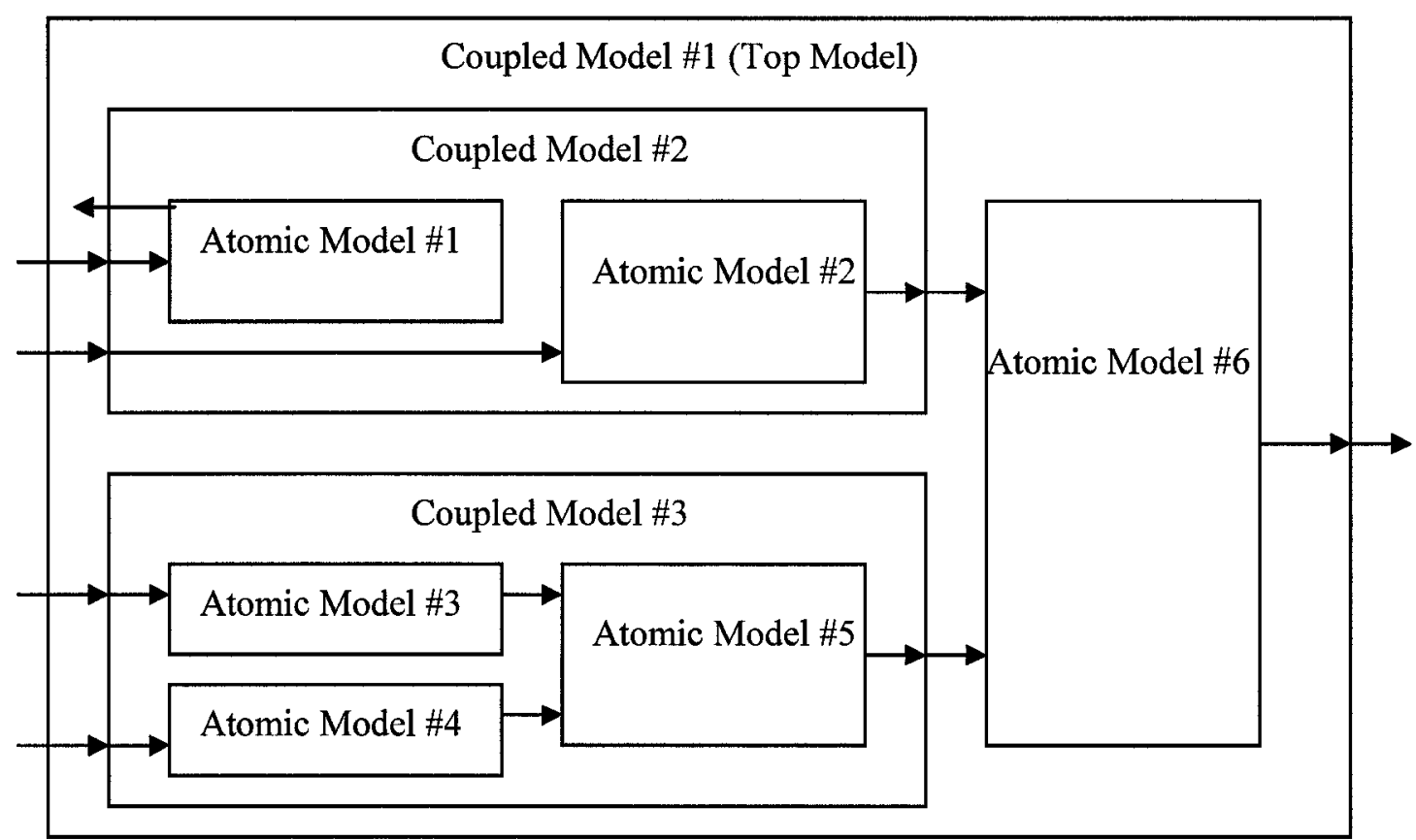

Figure 2: Structure of a Coupled DEVS Model

The Cell-DEVS formalism [8] is an extension to the DEVS formalism and is used to model real life systems that can be represented as cell spaces. Under Cell-DEVS, each cell in a cell space is represented as an atomic DEVS model. Each cell has input and output ports that are connected to its neighbouring cells or other DEVS models outside 
the cell space. The inputs to a cell can cause its state to change, in which case the new state will be transmitted to the neighbouring cells. In addition, a delay mechanism in each cell (transport delay or inertial delay) is used to delay the propagation of state change events through the cell space and thus provides the modeller a means to define complex temporal behaviour. The following is the formal definition of the Atomic CellDEVS [8]:

$$
\mathrm{TDC}=\left\langle\mathrm{X}, \mathrm{Y}, \mathrm{I}, \mathrm{S}, \theta, \mathrm{N}, \mathrm{d}, \delta_{\mathrm{int}}, \delta_{\mathrm{ext}}, \tau, \lambda, \mathrm{D}>\right.
$$

$\mathrm{X}$ is the set of external input events;

$\mathrm{Y}$ is the set of external output events;

I represents the definition of the model's modular interface;

$\mathrm{S}$ is the set of possible states for a given cell;

$\theta$ is the definition of the cell's state variables

$\mathrm{N}$ is the set of values for the input events;

$\mathrm{d}$ is the delay of the cell;

$\delta_{\text {int }}$ is the internal transition function;

$\delta_{\text {ext }}$ is the external transition function;

$\tau$ is the local computing function;

$\lambda$ is the output function, and

$\mathrm{D}$ is the duration function. 
Connecting the Atomic Cell-DEVS model together into a cell space produces a Coupled Cell-DEVS model. This model can take any shape, and can form a 2-Dimensional or a 3Dimentional cell space. The borders of the cell space can be either wrapped, in which case the cells at the border from one side of the cell space are considered neighbors to the cells at the border on the opposite side of the cell-space, or non-wrapped, in which case the border cells must have special rules defined by the modeler. The formal definition of the Coupled Cell-DEVS is as follows [8]:

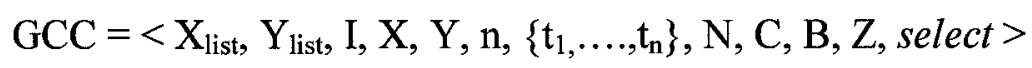

$\mathrm{X}_{\mathrm{list}}$ is the input coupling list;

$\mathrm{Y}_{\text {list }}$ is the output coupling list;

I represents the definition of the model's modular interface;

$\mathrm{X}$ is the set of external input events;

$\mathrm{Y}$ is the set of external output events;

$\mathrm{n}$ is the dimension of the cell space;

$\left\{t_{1}, \ldots, t_{n}\right\}$ is the number of cells in each of the dimensions;

$\mathrm{N}$ is the neighborhood set;

$\mathrm{C}$ is the cell space;

$\mathrm{B}$ is the set of border cells; 
$\mathrm{Z}$ is the translation function; and

select is the tie-breaking function;

\subsection{The Experimental Frame}

In the DEVS M\&S field, the System-Model-Simulator view is a very well accepted framework. It defines the relationships between the system, the model and the simulator: the system is related to the model by the modeling relationship and the model is related to the simulator by the simulation relationship. When a model is built for a system, the system is studied within a given context to derive the specifications of the model. Therefore, a model is valid only within this context and is meaningless without it [3]. It is therefore important to capture the specifications of the context in which a system is studied. It is generally agreed that the characterization of the context must explicitly state the underlying objectives, assumptions, and constraints of the study [3].

The Experimental Frame (EF) concept has been introduced to capture the set of circumstances under which a real system is to be observed, or capture the set of circumstances under which a model is to be subjected to experimentation[2][9] . This makes the meaning of EF ambiguous in that it could mean different things within the M\&S process. In [10] the idea of a framework that takes into account the different meanings of an EF was introduced. These ideas were formalized by the Context-FrameExperimentor framework and formalized by Traore and Muzy [3] . This framework, 
shown in Figure 3, presents a clear distinction between the three levels of abstraction, namely: the context through which a real system is being studied, the specification of this context as an $\mathrm{EF}$, and the implementation of this $\mathrm{EF}$ to execute on a simulator.

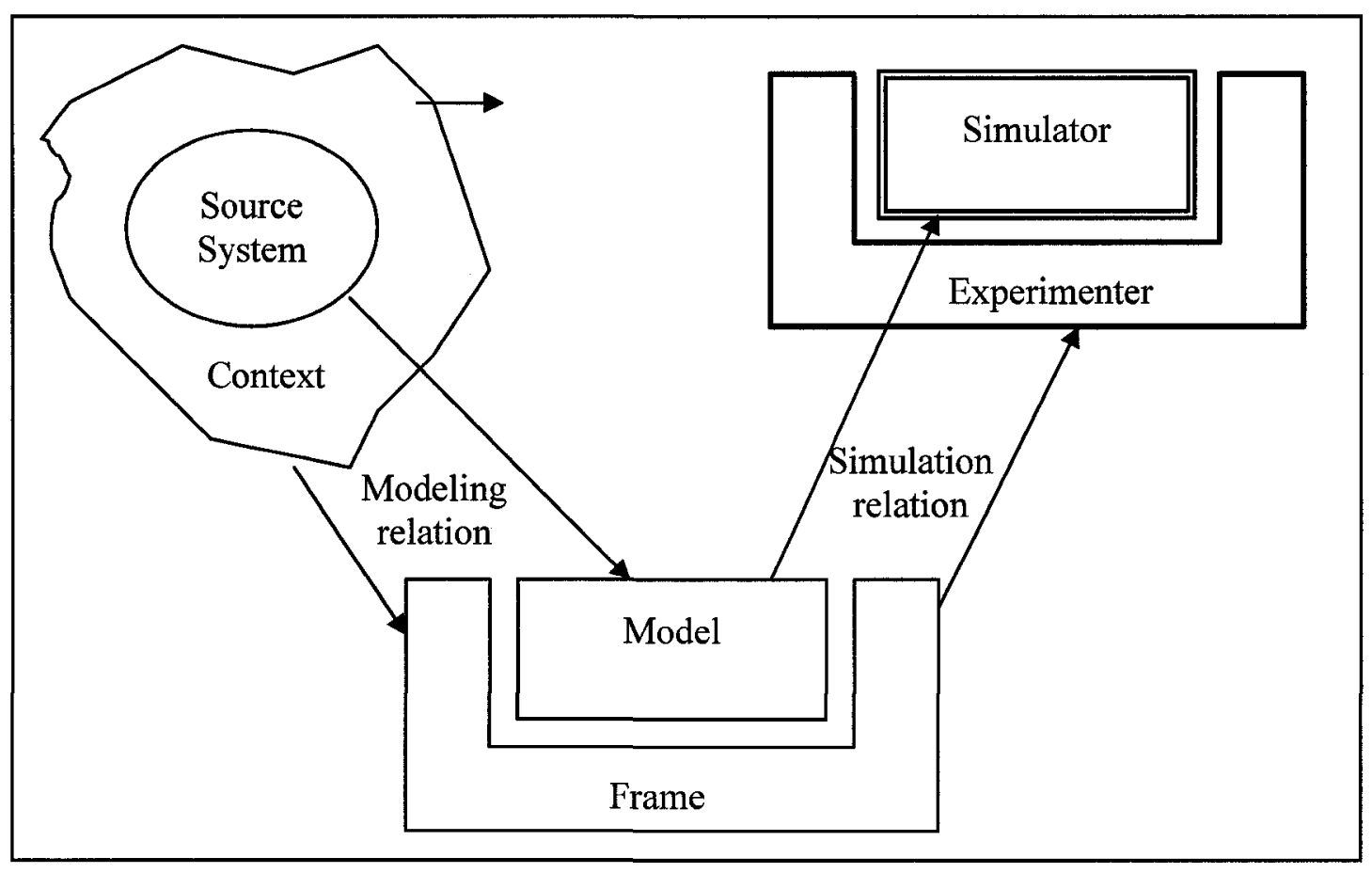

Figure 3: Modeling and Simulation Framework [10][3]

As mentioned earlier the context within which a system is studied during the modelling process is important to capture because the model is meaningless without its context. A specification of the context would also make it possible to identify models that may be relevant for re-use within a given context [3]. Using the framework of the experimental frame described in Figure 3, Traore suggests that the context be formally described by the $\mathrm{EF}$. This formal specification of the EF is described below. 
As shown in Figure 4, one can think of the EF as a circuit board with input and output ports into which a source system or a model can be 'plugged-in'. The EF itself can be a complex combination of components with their own interconnections and inputs and outputs. A simple example of an experimental frame is one made of three components, a generator, acceptor, and transducer as presented in [3]. Note that these EF components can be DEVS models in themselves. A subset of the inputs and outputs between the model and the EF form an interface to which both the model and EF must adhere.

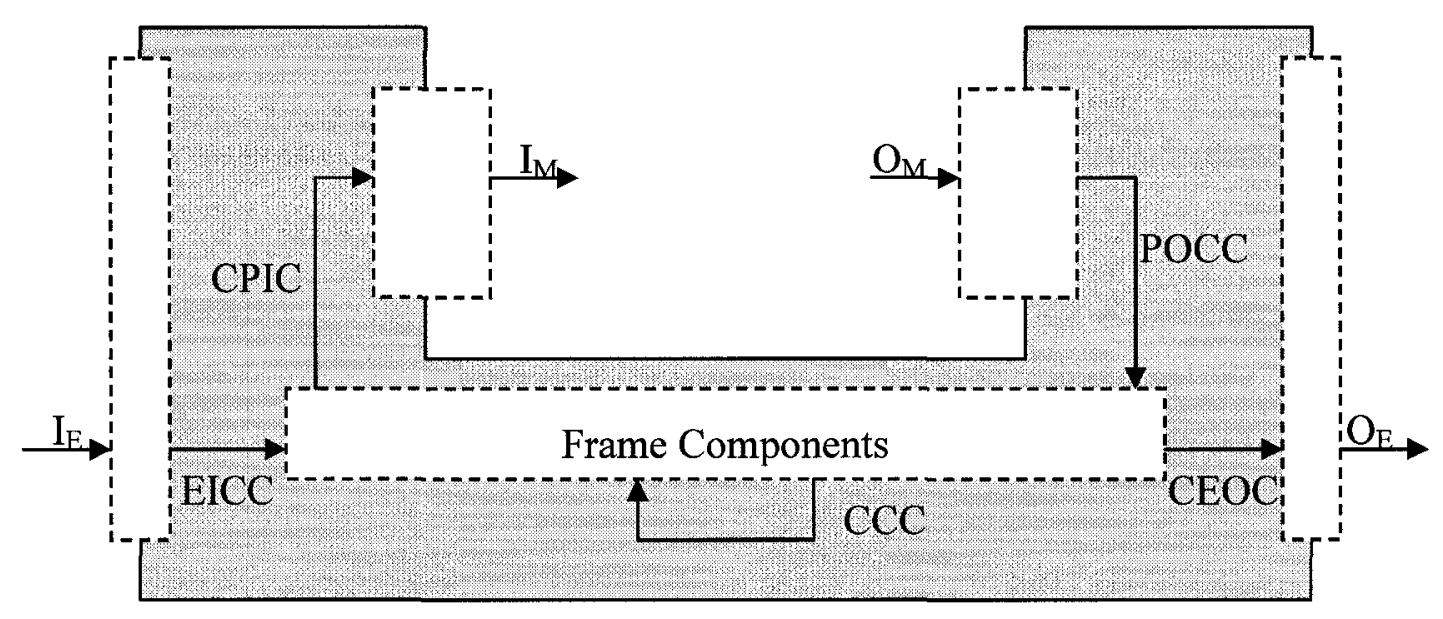

Figure 4: Experimental Frame System Specification [3][10]

The formal definition of the EF is [3]:

$$
\begin{gathered}
\mathrm{EF}=<\mathrm{T}, \mathrm{I}_{\mathrm{M}}, \mathrm{I}_{\mathrm{E}}, \mathrm{O}_{\mathrm{M}}, \mathrm{O}_{\mathrm{E}}, \Omega_{\mathrm{M}}, \Omega_{\mathrm{E}}, \Omega_{\mathrm{C}}, \mathrm{D},\left\{\mathrm{C}_{\mathrm{d}}, \mathbf{d} \varepsilon \mathrm{D}\right\}, \\
\text { CPIC, EICC }, \text { POCC }, \text { CEOC }, \mathrm{CCC}>,
\end{gathered}
$$

Where

$\mathrm{T}$ is a time base; 
$\mathrm{I}_{\mathrm{M}}$ is the set of Frame-to-Model input variables, the plug-in input set;

$I_{E}$ is the set of Frame input variables, the control input set;

$\mathrm{O}_{\mathrm{M}}$ is the set of Model -to-Frame output variables, the plug-in output set;

$\mathrm{O}_{\mathrm{E}}$ is a set of Frame output variables, the summary set;

$\Omega_{\mathrm{M}}$ is the set of admissible input segments for the plug-in component, the plug-in input constraints set;

$\Omega_{\mathrm{E}}$ is the set of admissible input segments for the experimentation control, the control input constraints set;

$\Omega_{\mathrm{C}}$ is the set of admissible output segments expected from the plug-in component, the plug-in output constraints set;

$\mathrm{D}$ is a set of component names, the control components set;

$\mathrm{C}_{\mathrm{d}}$ is a model for each $\mathrm{d} \varepsilon \mathrm{D}$;

CPIC is the Control-to-Plugin-Input coupling;

EICC is the External-Input-to-Control coupling;

POCC is the Plugin-Output-to-Control coupling;

CEOC is the Control-to-External-Output coupling;

$\mathrm{CCC}$ is the Control-to-Control coupling; 


\subsection{Reusable Modelling Components and Reusable Model Libraries}

The use of re-usable components in the modeling and simulation field is of interest to many researchers. In addition, the idea of creating a library of these reusable modeling components is attractive to many researchers since such a library would greatly facilitate the actual reuse of these components. In this section several research works in this area are presented.

In [7][11][12][13] the authors introduce an object-oriented architecture for the definition of web-based hierarchical models libraries. This architecture is based on [12]: The notion of being a generic library, the ability to manage abstraction and inheritance between stored models, and the ability to access the library using web-based procedures. By being a generic library the authors propose to store models in the library in what is called a "context-out" format. When a model is extracted from the library it has to be converted to its "context-in" format to be able to use it in a specific simulation environment. The authors focus on structuring the models in the library according to their domains and the abstraction levels. The domain refers to the theoretical domain of the stored models (for example DEVS, VHDL ... etc). A subset of the domain is an application domain which refers to the actual application domain for a given model (for example Microelectronics). The abstraction level on the other hand refers to the amount of information contained in a given model. 
In [14] the authors are concerned with reducing the knowledge gap between the creators of behaviour models (the analysts) and the users of those models (designers). This paper focuses on proposing a meta-data representation to characterize behavioural models. This meta-data gives the model users information such as the assumptions, limitations, and context of the models. Based on this a prototype repository is presented to store the models and the meta-data. The repository is envisioned as a store of behavioural models from which model designers select a single model that is most appropriate for their objectives, then they populate the model with their specific design parameters, and finally they execute the model and evaluate the results, refining the parameters and re-executing if needed.

In [15] the authors propose developing a library of reusable model fragments and models. A large part of this paper is concerned with proposing a structure for the models that go into the library. The proposed structure divides a models into three "viewpoints", namely a technical component viewpoint (e.g. a resistor), a physical concept viewpoint (e.g. resistance, weight ...etc.), and mathematical viewpoint. The library stores each of these viewpoints separately in the library and a complete model is created by linking these model fragments together. Complete models can also be stored in the library. In addition, to facilitate the management and use of models, the documentation for them is stored, they are categorized into a taxonomy structure, and their quality information is stored. 
Building a model using this library involves specifying all three viewpoints for it and adding a model to the library involves a "library manager" creating links to it so that future users can find it. The library does not store actual model files directly but uses a "generic description language" that is then exported to the format of the simulation tool being used (such as Matlab, Simulink ... etc). The paper does not specify this language or how it is exported.

In [16] the authors present a very general wish-list of what their envisioned repository would do with no details of design or architecture. The repository is envisioned to contain models from various categories such as networking models, discrete simulation models, linear and non-linear models... etc. The repository is intended for use in higher education setting where students and faculty could use the models to apply knowledge learned in courses to real world problems. It is clear that at the time of publishing of this paper, the authors had not yet designed the structure of the repository or the elements stored in it.

In [17] the author creates a collection (library) of reusable models and shows how they can be used in the Visual Simulation Environment (VSE) for visual simulation of the NCSTRL system. The paper demonstrates how the reusable components can be used to create simulations for different configurations of the system without doing any code changes. The paper however does not present an actual library architecture; but it refers to the collection of nine re-usable models that were created as a library of re-usable 
models.

In [18] the authors do not actually propose an architecture of a model library. This paper simply takes a specific example, "the flow of jobs through a job shop", and from it extracts requirements information for each component of that example (this is information specific to the example). Then the paper goes on to create a number of database tables based on this information; thus, these tables are also very specific to the example. Finally, a model builder is created which is capable of taking the "neutral' information from the database and converting it into a simulation model that can be run. The database tables created in this work are specific to an example and cannot be used as a basis for a general model library.

In [19] the authors focus on using JavaBeans to create reusable simulation components. This paper presents guidelines for building reusable component architectures and then demonstrates the use of these guidelines through several component architectures. The paper is not concerned with building a library in which to store the reusable components.

There are also the following simulation platforms that should be mentioned here, namely NS-2 [20], OMNeT++ [21], and OPNET Modeler [22]. These are all simulator platforms that are focused on the networking field. They provide users with a collection of network components from which users can build the network to be simulated. They also provide 
the ability to create network components that can then be shared with other users. OMNeT++ for example currently has on their website a list of 41 "Supported Models" and 24 "Contributed Models". NS-2 also has a website with a set of links to "modules" contributed by users. Being a corporate product, OPNET Modeller comes with a large suite of components that enable users to model many types of networks. Other than the listings on the websites, there does not seem to be a publicly available repository of models that is capable of managing the stored models.

\subsection{DEVS-Based Simulation Toolkits}

The $\mathrm{CD}++$ Builder toolkit is one of many different implementations of tools that enable modeling and simulation based on the DEVS formalism. Most of these tools are concerned with providing an engine to run DEVS simulations on and with providing a library of classes upon which modelers can build their DEVS models. These tools do not provide a way to store DEVS models for future reuse.

A list of some of the other DEVS simulation tools is available on the internet at [23]. The following is a brief list of a selection of some of the tools listed on this website with a short description of each. It is worth noting that none of these toolkits has capabilities similar to the capabilities of the $\mathrm{CD}++$ Repository, which will be introduced in later chapters. 
- ADEVS [24]: This tool provides a C++ class library based on the Parallel DEVS and DSDEVS formalisms which developers can use to build their own models. It includes support for standard, sequential simulation and conservative, parallel simulation on shared memory machines with POSIX threads.[23]

- DEVS/C++ [25]: This is a DEVS simulation environment based on the ParallelDEVS formalism and implemented in the $\mathrm{C}++$ language. [23]

- DEVS/HLA [26]: This is a High Level Architecture (HLA) compliant DEVS environment. It was developed to demonstrate how an HLA-compliant DEVS environment can significantly improve the performance of large-scale distributed modeling and simulation exercises. [23]

- DEVSJAVA [27]: This is a DEVS modeling and simulation environment implemented in JAVA. It supports parallel execution on a uni-processor and supports higher-level, application specific modeling. [23]

- JDEVS [28]: This is a Java based DEVS modeling and simulation environment that enables general purpose, object oriented, component based, GIS connected, visual simulation model development and execution. [23]

- SimBeans [29]: This allows component based DEVS modeling and simulation on the basis of Java and JavaBeans. [23] 


\section{Chapter 3: Problem Statement}

The DEVS formalism provides a framework for building modular and hierarchical simulation models. This is a first and important step towards the goal of sharing and reusing modeling components. However, one of the main problems that stand in the way of the reuse of DEVS models is the inability to share DEVS models between model designers in an easy and correct fashion. A mechanism is needed by which model designers can access and contribute DEVS models to a pool of shared models. Nevertheless, the ability to access other designers' DEVS models alone is not enough to enable reuse. This is because a model is useless without the knowledge of how it can be used and in what context. Therefore, a mechanism is also needed to enable sharing of the conceptual information describing the DEVS models; this information must include information about the context within which the model was intended to be used. In addition, model designers could have created a number of experiments to test their DEVS models. Sharing these experiments and their results provides other users with valuable information about the workings of the DEVS model as well as a measure of confidence in the accuracy of the DEVS model. The ability to rerun an experiment and reproduce the same simulation results and the availability of the simulation logs to new users are important to ensure fidelity of the models especially in a publicly available system where anyone can contribute models and experiments. Therefore, a mechanism that allows sharing and reuse of these experiments and experimental results is also required. 
It follows that the aim of this work is to develop a mechanism that facilitates the sharing and reuse of DEVS models, their experiments, and their experimental results. Chapter 2 described a number of previous research efforts that aimed at creating repositories of reusable simulation components. Nevertheless, each of these research efforts emphasised a specific problem and presented their version of a model library as a solution to it. As will be shown in the next few paragraphs, none of these solutions provides all of the elements desired in a DEVS model repository.

To start, the works presented in [16], [17],[18], and [19] do not present an architecture of a model repository that can be used to store and retrieve modelling components. Instead [16] is a brief paper with a very general description of the author's vision of what a model repository would do. In [17], [18], and [19] on the other hand the author's use the term 'library' to refer to the collection of models that they are presenting in their works, and that could in themselves be reused, but do not present an infrastructure to manage these models. Thus, these works are not suitable to solve the problem at hand.

Next, the simulation platforms NS-2, OMNeT, and OPNET Modeler discussed in Chapter 2 are focused on the networking field and thus provide components that are primarily for modelling of networks. In addition, they do not have a repository of models as such; instead, OPNET Modeler provides its models as part of a package when users purchase the product and thus its models are not open for public use, while NS-2 and 
OMNeT provide listings of their models on their web pages. This makes locating models of interest to a user a difficult task especially since no "context of use" information is provided up front. Furthermore, no mechanism is provided for sharing experiments and experimental results. Thus, the libraries of models provided by these platforms are not suited for use as a publicly available repository of models and experiments.

Next, the authors of [15] present a structure for a library of reusable models. However a main focus of this library is on assisting the user in the building of the low level details of the modular components themselves (by specifying mathematical viewpoints, etc), which complicates the process of using the library and is not necessary for a library of reusable models. In addition, this library relies on a set of pre-existing technical components to use for building other components, meaning that the library is built for a specific field and is not general enough to be used for models from different fields. Furthermore, building and maintaining the taxonomy structure of the technical components in the library requires a modeller experienced in the specific field who can recognize the links between the different components. This hinders the ability to add modelling components that could conceivably be from any modelling field (since you would need experienced modellers in every field to maintain the library). As a final note, the library does not provide a means for storing and linking the experiments and experimental results pertaining to the stored models. Thus, this model library is also not suited for use as a publicly available repository of models and experiments. 
Next, the authors of [14] propose a meta-data representation to describe the behavioural models' assumptions, limitations, accuracy and context. Then they propose a prototype repository to store the models and their meta-data. This sounds very promising except that it does not allow the storage of a hierarchy of linked models (such as coupled DEVS models). It assumes that the stored models are in one piece and are not built from other smaller modular components. This will not work for DEVS models since they are hierarchical in nature and a Coupled DEVS model is ultimately made up of child Atomic models. When a coupled model is stored in a repository all the child atomic models should therefore become part of the repository as well, which is not the case here. As a result, this repository would not be very useful for storing coupled DEVS models. In addition, the repository proposed in this paper does not contain a mechanism for storing and reusing experiments and experimental results. Having these experiments and experimental results is important for ensuring model fidelity in a publicly available repository. Thus, this model library is also not suited for use as a publicly available repository of models and experiments.

Finally, in [7][11][12][13] the authors introduce an architecture for creating web-based hierarchical model libraries. The library proposed in these papers is capable of managing inheritance and abstraction links between stored models. The library can be consulted to find the model of interest, however there does not seem to be a facility to search the 
library. Instead the models are categorized according to domains and sub-domains and the abstraction levels. In addition, other than the domains and the abstraction links, there dose not seem to be descriptive or context of use information for the stored models. Finally, the repository proposed in this paper does not contain a mechanism for storing and reusing experiments and experimental results. Thus, this model library is also not suited for use as a publicly available repository of models and experiments.

As a result, none of the existing solutions is suitable to solve the problem of model sharing and reuse. In this work, we define new mechanisms for accessing DEVS models, their context of use information, their experiments and experimental results. We propose new methods for linking the stored items together as appropriate (for example linking a model to its experiments). The prototype software application is publicly available on the internet such that any user can add to or download items from it following an opensource based approach. Finally, it provides information (including context-of-use information) to enable users to locate models and/or experiments of interest to them.

The following table summarizes the capabilities of the existing model library architectures presented in Chapter 2 compared to the capabilities of the proposed DEVS model repository. 


\begin{tabular}{|l|c|c|c|c|c|c|c|}
\hline & $\begin{array}{l}\text { This } \\
\text { Work }\end{array}$ & $\begin{array}{l}{[7],[11],} \\
{[12],[13]}\end{array}$ & {$[14]$} & {$[15]$} & NS-2 & OMNeT & OPNET \\
\hline Stores Models & Yes & Yes & Yes & Yes & Yes & Yes & Yes \\
\hline $\begin{array}{l}\text { Stores context-of- } \\
\text { use information }\end{array}$ & Yes & Yes & Yes & Yes & No & No & Yes \\
\hline $\begin{array}{l}\text { Stores reusable } \\
\text { experiments }\end{array}$ & Yes & No & No & No & No & No & No \\
\hline $\begin{array}{l}\text { Hierarchical } \\
\text { construction }\end{array}$ & Yes & Yes & No & Yes & No & No & Yes \\
\hline $\begin{array}{l}\text { General (i.e. not } \\
\text { limited to a } \\
\text { specific field) }\end{array}$ & Yes & Yes & Yes & No & No & No & No \\
\hline $\begin{array}{l}\text { Accessible over } \\
\text { the internet }\end{array}$ & Yes & Yes & Yes & No & Yes & Yes & No \\
\hline Searchable & Yes & No & Yes & No & No & No & Yes \\
\hline $\begin{array}{l}\text { "Open-Source" } \\
\text { concept }\end{array}$ & Yes & No & No & No & Yes & Yes & No \\
\hline
\end{tabular}

Table 1: Comparison with Exiting Model Repositories 


\section{Chapter 4: Architecture of the Repository}

The architecture presented in this chapter can be used to implement a repository for any of the DEVS tools in existence today. As discussed in Chapter 3, this work intends to enable the creation of a repository of DEVS models that is capable of managing models, experiments, experimental results and their conceptual information in such a way that facilitates their reuse. The repository must be accessible from different geographical locations enabling users to either contribute their DEVS models and/or experiments to the repository, or search for and download the DEVS models and/or experiments from the repository. The repository is to store the conceptual, descriptive, and context of use information for each model in order to make possible the sharing of the models in a useful way.

To achieve this, the repository design uses a client-server architecture (with all of the information stored in a central database server that is accessible by the repository's client application over the Internet). The DEVS models are stored in the repository as atomic and coupled entities each with their own descriptive information and model files. The coupled entities are linked to their atomic/coupled sub-components thus preserving the natural hierarchical structure of DEVS models, and enabling the efficient and modular storage of the models. Each model entity is linked to an Experimental Frame (EF) entity in the database. EF entities include information about the context of use for the linked model, as well as any number of experiments that are applicable to that model. The 
experiments are stored as entities of their own with their own descriptive information and their own experiment files. This enables the linking of a given experiment to more than one model thus enabling the re-use of the experiments. Experiments could be of two types either "event-based" or "model-based". Event-based experiments use a file to drive the inputs of the model for the experiment, while model-based experiments use other DEVS models as generators and transducers. In the case of model-based experiments, the DEVS models used as generators and transducers are also linked to the experiment entity. The last major entity stored in the database is the experimental results entity, which is composed of descriptive information and files generated by a run of the experiment. The experimental results are put into separate entities from the experiments because a given experiment could be linked to more than one model; each experimental result is therefore associated with a model-experiment pair. Other entities stored in the repository include input/output and range entities used to specify the inputs and outputs of the EF (as per the EF formalism presented in Chapter 2).

From a very high level, the proposed repository is comprised of two main components; a server and a client component. The server component contains a central database containing the models, their experimental frames, experiments, and experimental results. The database is hosted on a computer connected to the internet that the client component can connect to. The client component is responsible for querying the database and presenting results to the user, as well as adding new entries into the database at the 
request of the user.

The heart of this architecture is in the details regarding the stored entities. The next subsections will take a closer look at the storage engine, explain the exact entities that it is able to store, the information stored about each entity, and the relationships between the stored entities.

\subsection{The Storage Engine}

In essence, the repository is a store of DEVS Models and their Experimental Frames (which include the actual experiments). Therefore, at its core there is a storage engine capable of storing and retrieving all of the relevant information. As with most other database applications, a relational database management system (DBMS) was chosen as the core of its storage solution. Relational databases are proven to be reliable and easily support the tasks of querying and adding items. In addition to the relational database, the storage engine uses an FTP server to store files related to the stored entities. Both the database and FTP Servers reside on the same physical machine that is connected to the internet. As shown in Figure 5, client machines that are connected to the internet can connect to the Database and FTP servers in order to upload or download information. 


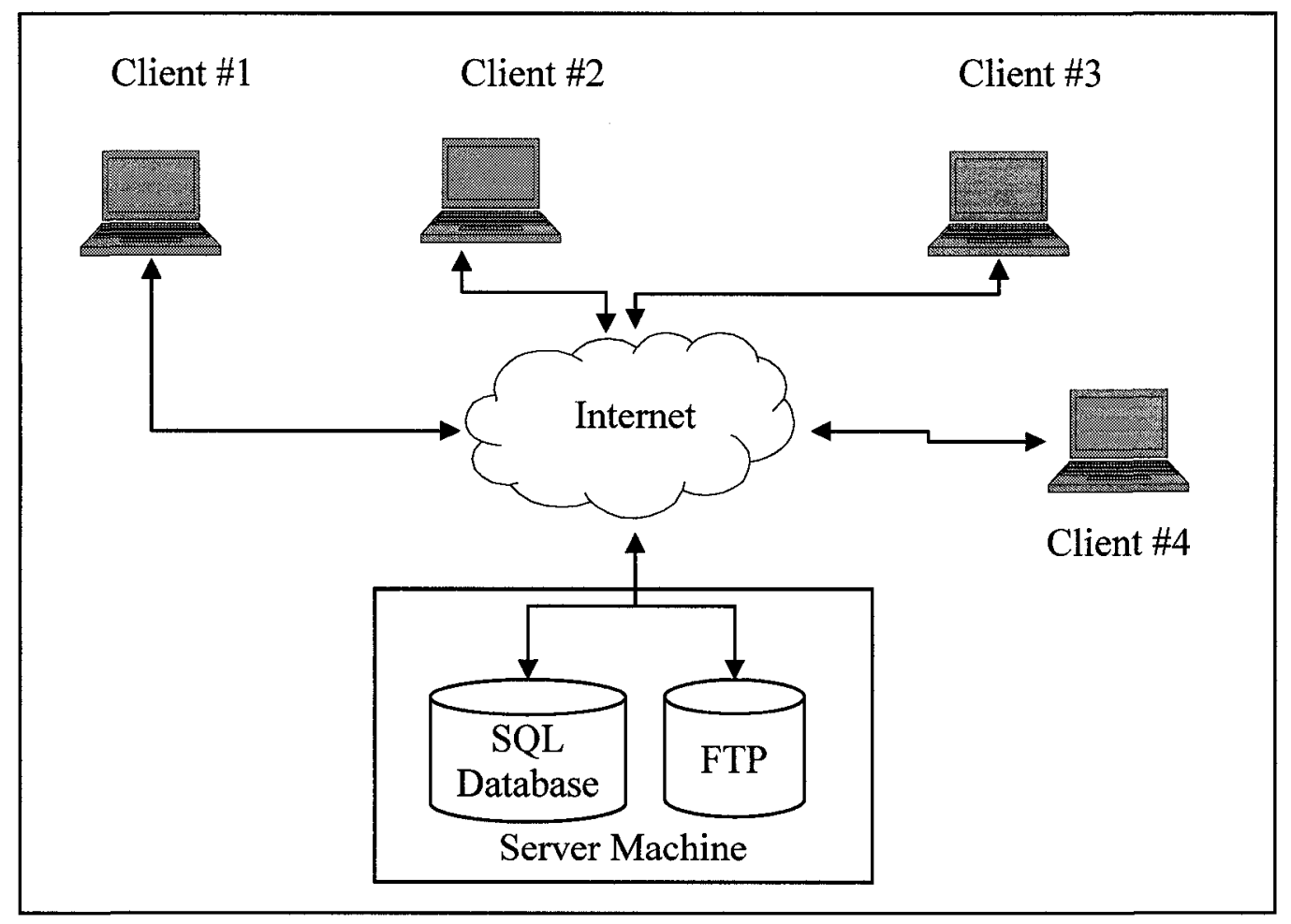

Figure 5: Repository Storage Architecture

The Relational Database Server is the heart of the storage system since it holds all of the information for all of the entities stored by repository. This includes all of the relationships between all of these entities. The entities stored in the Relational Database are Atomic Models, Coupled Models, Experimental Frames (including Experimental Frame Inputs and Outputs, and Ranges), Experiments, and Experimental Results. A detailed discussion of what these entities are actually composed of and what information is stored for each is presented in the following sub-sections. 
Each of the model entities as well as the experiment and experimental result entities may have a number of files related to them. For example the Atomic Model may have a $\mathrm{C}++$ program file (.cpp), a header file (.h), a model definition file (.ma), and a description document (.doc); in fact this is the case for $\mathrm{CD}++$ Atomic models as will be seen in later chapters. These files are compressed into a (.zip) file and stored on the FTP server. Each entity in the Relational database has a "pointer" to the (.zip) file that concerns it on the FTP server. Thus, the FTP server can be seen as a dumb store of files, while the Relational Database Server contains the information describing each entity and relating it to the other entities in the system and to its (.zip) file on the FTP Server.

\subsection{The Entities Stored in the Repository}

The goal of this repository architecture is to enable sharing of DEVS models and experiments among users. Therefore, the information pertaining to these models and experiments has to be encapsulated and stored in the database. This leads to the creation of the storage entities that have been mentioned in the previous section. In the following paragraph, the rational behind deciding on these storage entities is presented.

It is clear that a model storage entity is required to store the DEVS model information; more specifically however, in DEVS there are two basic types of models Atomic and Coupled. Therefore, both atomic model and coupled model storage entities are required. 
However, as mentioned earlier, a model without its context is meaningless; therefore an entity is needed to hold the context of use information for the model. A look back at the Experimental Frame idea presented in Chapter 2 leads to the creation of the Experimental Frame (EF) storage entity. This entity will hold the information about the context within which the model is valid. Each model entity has one EF entity linked to it. The next item missing from the repository is the experiment. On first thought, one might be tempted to include the experiment information within the EF entity. If that were done however, it would not be possible to share experiments among different models. In a real life scenario, experiments can in fact be reused to test different DEVS models; therefore, the storage architecture should reflect the fact that a number of models can share one experiment. As a result, an experiment storage entity is required to hold the experiments' information. Then, these experiment entities can be linked to any number of EF entities to represent the experiments that belong to each EF entity. The final missing item is the experimental results. Experimental results are obtained by running a given experiment on a given model, and thus by definition they refer to a model-experiment pair. As a result an experimental results entity is needed to store information relating to them, this entity is linked to both a model entity and an experiment entity.

The conceptual meaning of these entities is clear from the above discussion, but to give an exact meaning to these entities one needs to specify what information is actually being stored for each. What are the pieces of information that need to be stored by the 
repository for each entity, and how can the relationships between all of these entities stored? These questions are central to the creation of the repository. The following subsections describe each of these entities in more detail, and answer the aforementioned questions for each.

\subsubsection{Atomic and Coupled DEVS Models}

In general, DEVS models can be either Atomic or Coupled Models, with Cell-DEVS models being a special case of the Coupled model. So far the DEVS models have been discussed as abstract entities or as a mathematical formalism (as in Chapter 2), however to be able to describe a DEVS model to a simulation tool the model is usually describe programmatically via a computer program or some other format that a computer can understand. Thus, for each model storage entity a number of files describing the model should be stored. The exact files depend on the exact simulation tool for which the repository is being designed. In addition, Atomic and Coupled models may require different kinds of files. As an example, the CD++ Builder Toolkit represents an Atomic Model with three files, the first two are the $\mathrm{C}++$ class files (program (.cpp) and header (.h) files), the last file is the model definition file (.ma) containing the coupling scheme for the model as well as some other parameters. Coupled Models (including Cell-DEVS models) on the other hand are described using only one file, the model definition file (.ma). In this file a specially designed specification language allows the description of all 
the components (sub-models) of the Coupled Model and allows the declaration of the interconnections of the input and output ports of all of these sub-models. In addition to these that are specific to the simulation tool, a file that contains a detailed description of the model, a (.doc) file for example, is useful for users to understand the exact details of the model, and is thus required for storage by the repository. To accommodate the fact that each simulation tool has its own set of files that represent the models, the architecture of the repository specifies that each model entity, be it atomic or coupled, should store a pointer to a location on the FTP server where all the required files may be found. This location may be a directory, a (.zip) file or any other convenient location.

In addition to files however, the model storage entity should also store some data describing each model. This data describes the model in a way that is useful to a human user so that when the user is presented with this data for a number of models, he/she can quickly determine the model that interests them. The simplest example of such data is giving a name to each model. There are many other candidate pieces of information that can be stored with the models to more fully describe them. This information will be referred to as the Model Data throughout this thesis. The Model Data is comprised of the following pieces of information:

1. Model Name: This is a unique name generated by the repository software for each model in the repository.

2. Domain: This is the domain under which the model can be categorized. Examples 
are Telecommunications Equipment, Urban Traffic.... etc.

3. Title: Is a descriptive title for the model.

4. Acronym: Is the acronym for the model.

5. Brief Description: This is a short paragraph (less than 250 chars) describing the model's general characteristics.

6. Key Words: These are a few key words that are associated with the model, and that can be useful when doing a search of the repository.

7. Developer Name: The name of the model's developer.

8. Date Developed: The date the model was developed.

In the relational database, the model entity can be translated, roughly, into a database table with the Model data comprising the majority of its columns. This allows the repository the ability to search for models based on the information provided in the Model Data. For example, a user can search for all models in the repository with a Domain of "Telecommunications Equipment", or for all models with the word "motor" in their Title.

So far, the discussion of the DEVS models stored in the repository ignored the fact that, by definition, Coupled DEVS models have 'relationships' with other models in the 
database. As explained in Chapter 2, a Coupled Model is composed of a tree of other 'child' DEVS Models that could themselves be either Atomic or Coupled. This hierarchical construction of Coupled DEVS models is one of the advantages of the DEVS formalism. When a Coupled model is stored in a repository of models it could simply be stored as one entity (a generic model entity as has been described so far) containing its own files and all of its children's files. However, by doing that one will lose the advantage of the hierarchical construction built into the DEVS Coupled model. Therefore, the proposed repository's architecture maintains the hierarchical construction of the Coupled models even while they are stored. This is done by creating separate atomic model and coupled model entities that are basically copies of the model entity described above. The coupled model entity can then be linked it to its tree of child model entities in the repository. For example, suppose a Coupled model A is composed of the two atomic models $\mathrm{X}$ and $\mathrm{Y}$, and another Coupled model $\mathrm{B}$ is composed of Coupled model A and Atomic model Z. Each of these models are stored separately in the repository, with Model A linked to Models X and Y, and model B linked to models A and $Z$ (See Figure 6). In this case, when the repository is asked to retrieve model B for download it is able to follow the links and retrieve all of model B's child models, thus retrieving all of the models in Figure 6. 


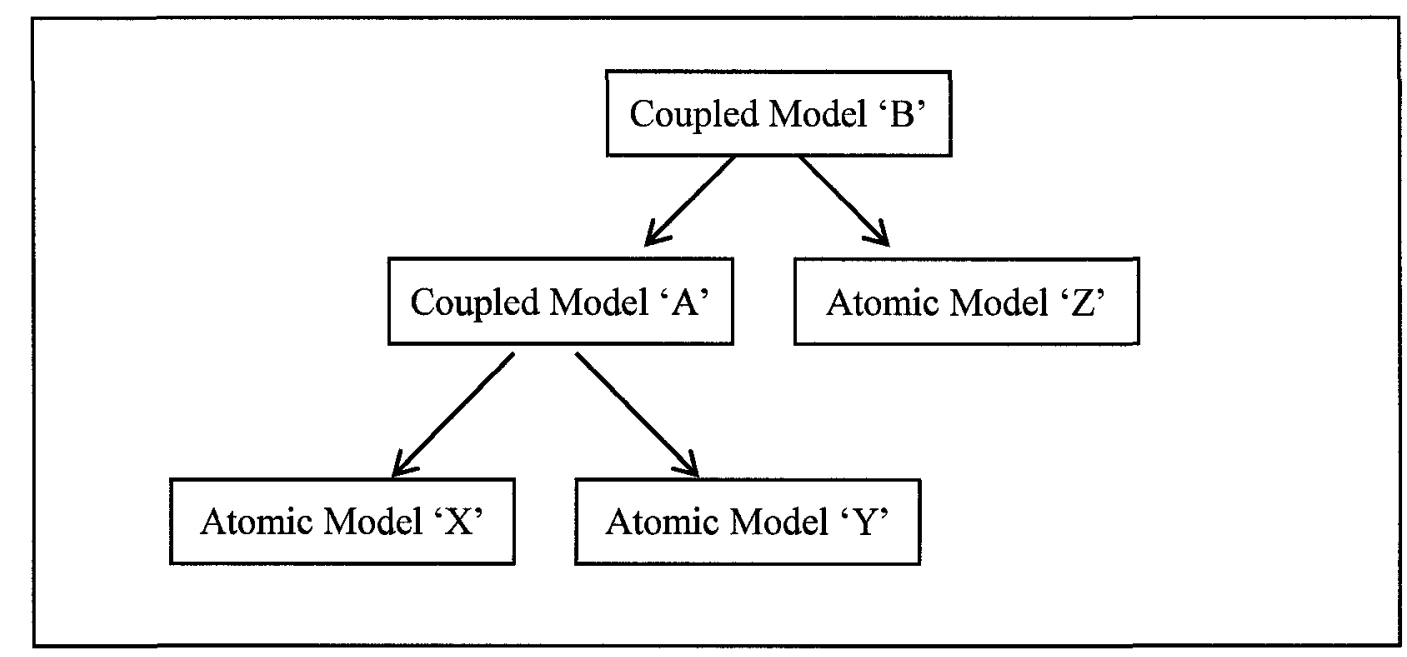

Figure 6: Structure of a Stored DEVS Coupled Model

\subsubsection{Experimental Frames}

While storing the DEVS Models themselves is a major part of this work, an important feature of it is the storage of an Experimental Frame for each stored Model. The Experimental Frame storage entity must always be linked to one model entity in the repository. The Experimental Frame entity should be able to store Experimental Frame Data (analogous to the Model Data), should be linked to a set of experiment entities that are related to the model (described in the next sub-section), and should contain other EF information corresponding to the formal definition of the Experimental Frame (described later in this section). Unlike the model entity, experimental frame entities do not have any files associated with them. In the relational database, the EF entity can be represented as a table with the Experimental Frame Data comprising most of its columns, and with links to a model entity, and a number of experiment entities. 
The Experimental Frame Data is the data used to describe the context within which a given model is valid. This data is displayed to the user as part of a model's descriptive information to assist them in making a decision as to the usefulness of that model to them. This data is comprised of the following pieces of information [3]:

1. Objectives: These are the objectives for which the model was built.

2. Assumptions: These are the assumptions made by the model designer when designing this model.

3. Constraints: These are the constraints within which the model was designed to operate.

As explained in Chapter 2 the experimental frame captures the context within which the model operates. Any real life scenario can be seen from different angles and more than one model can be created each having its own context (and thus its own Experimental Frame Data). For example, a forest fire might be modeled with different objectives in mind: one objective can be determining the rate of fire spread, while another objective can be determining the amount of pollution caused by the fire. This leads to two different models for the same scenario. Similarly, the assumptions and constraints can be different for different models modeling the same real life system each from their own context. A model's Experimental Frame captures this information, and in the repository, it is stored as the three pieces of data Objectives, Assumptions, and Constraints. 
The final part making up the Experimental Frame entity is what is referred to here as the other EF information. This information is composed of some of the data contained in the formal definition of the Experimental Frame. In Chapter 2 the formal definition of the EF was shown to contain the set of inputs and outputs between the EF and the model and between the external world and the EF. The formal definition also had sets of admissible values for each input/output. The proposed repository architecture is capable of storing this information for each Experimental Frame. This way all of the inputs and outputs of the experimental frame and the valid range of values for each input or output are captured. In addition, this information is stored in such a way as to eliminate any repetition inside the database; this is shown in Figure 7 below. The inputs and outputs are stored as separate entities (i.e. in a separate database table) and are linked to the ranges which are themselves also stored as separate entities. This allows more than one input/output with the same range values to share the same range entity simply by referencing it. Similarly, Experimental Frames can share the same input/output entity. This information can be used to search or sort models with similar EF by comparing the inputs, outputs, and ranges. 


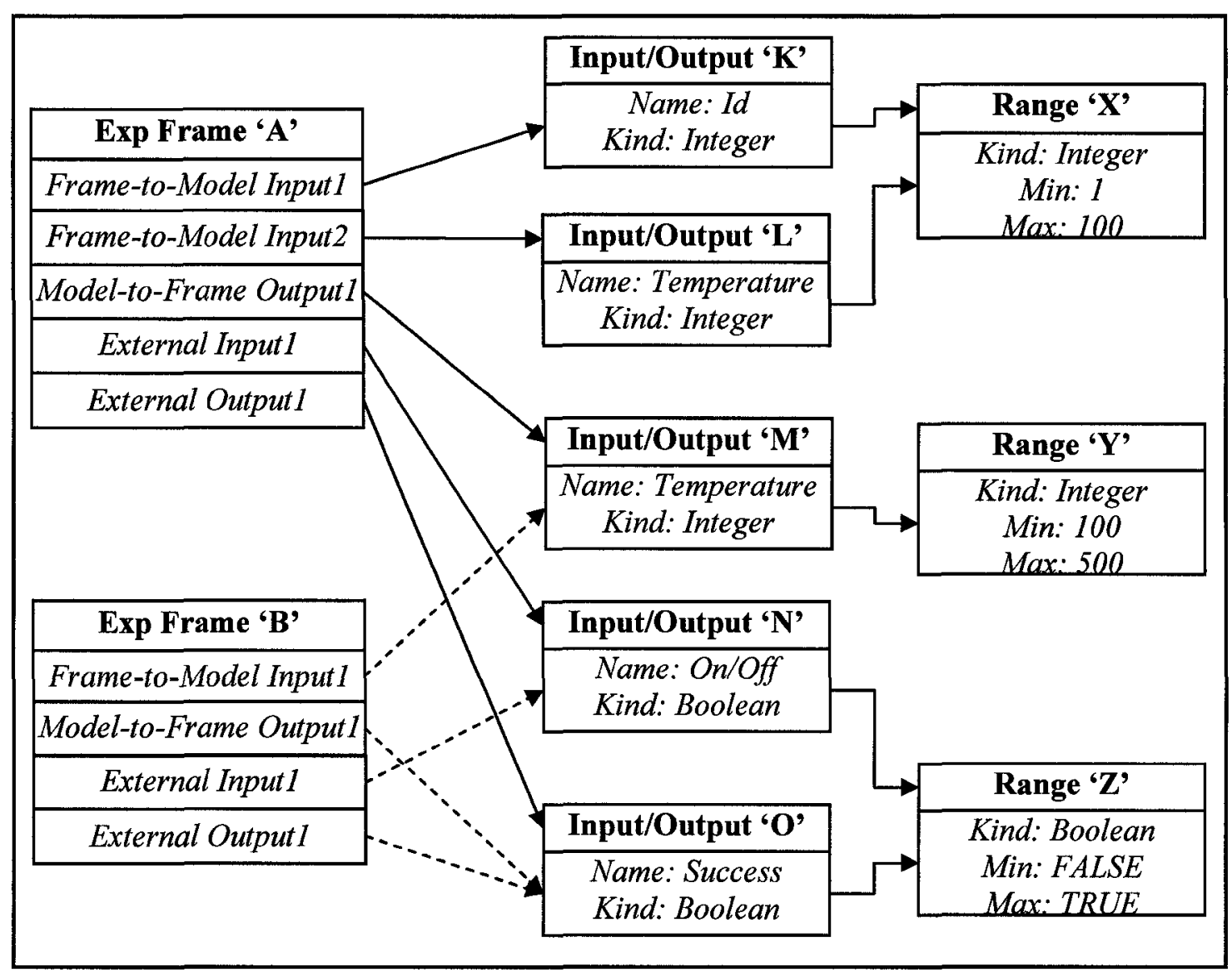

Figure 7: Structure of a Stored DEVS Coupled Model

\subsubsection{Experiments}

As mentioned in the previous section, the Experimental Frame entity is linked to a set of experiment entities that apply to the model to which the EF belongs. As mentioned earlier, a given experiment may apply to more than one model and thus may be linked to more than one EF. Each experiment entity is comprised of Experiment Data (analogous to the Model Data), and a pointer to a location on the FTP server where all the 
experiment files can be located. Similar to the model files, experiment files vary from one DEVS simulation tool to the next. In general, any files needed by the simulation tool for which the repository is being design should be included in the specified location on the FTP server. In addition, a file containing a detailed description document of the experiment should also be stored to assist users in understanding the details of the experiment. As an example, the CD++ Builder Toolkit can have the following experiment files event files (.ev), draw files (.drw), initialization files (.val), pallet files (.pal)...etc.

The Experiment Data is used as a textual description to be displayed to the user to assist them in choosing the experiments that suite their needs. In addition, the $\mathrm{CD}++$ Repository is able to search for experiments based on the information provided in the Experiment Data. The Experiment Data is comprised of the following pieces of information:

1. Experiment Name: This is a unique name generated by the repository software for each experiment.

2. Title: Is a descriptive title for the Experiment.

3. Brief Description: This is a short paragraph (less than 250 chars) describing the experiment in general.

4. Objectives: These are the objectives for which the experiment was created.

5. Assumptions: These are the assumptions made by the designer of the experiment. 
6. Constraints: These are the constraints within which the experiment was designed to operate.

7. Developer Name: The name of the experiment's developer.

8. Development Date: The date the experiment was developed.

It is important here to explain the difference between the objectives, assumptions and constrains of the experiment as opposed to the experimental frame. For the experimental frame, these pieces of information refer to the model and the characteristics of the model itself, however for the experiment they refer to the experiment itself and thus are usually a "sub-set" of those in the experimental frame. For example, a model of an elevator's motor could have an experimental frame with a constraint saying the maximum load in the elevator is $2000 \mathrm{lbs}$. At the same time, an experiment wanting to test the performance of the motor when empty would have a constraint saying the maximum load on the elevator is zero.

One last detail regarding the DEVS experiments is that, in general, they can be of two basic kinds: "event file" based experiments and "model" based experiments. The "event file" based experiments are ones where the input to the model is driven by an event file that contains a list of the values at the input ports of the model at specified times during the simulation. While the experiment is running, the input values in the event file are put 
at the appropriate time at each input port. By doing this one can test a specific scenario of inputs in each of their experiments and examine the output. On the other hand, "model" based experiments are experiments where the inputs to the model under test are generated by another DEVS model, a "generator". To clarify how this works assume that a user created a model for an oscilloscope and they want to create an experiment for their model. The user happens to know that there is an existing model of a signal generator that is established to be a good and accurate model. The user can link the signal generator to his/her oscilloscope model and use it as the input generator for the experiment instead of using event files. Similarly, the user could have another model linked to the output ports of the model they want to test where this model would act like a transducer of the outputs of the experiment.

The proposed repository architecture should support both of these kinds of experiments. To do so the experiment entity must allow the storage of an "event file" with the rest of the files, and must allow links to two model entities, one representing the generator and the other representing the transducer.

\subsubsection{Experimental Results}

The last piece of information maintained by the proposed repository is the experimental results for a run of a given experiment on a given model. The information stored for each 
experimental results entity is composed of a Boolean value to indicate success or failure of the experiment, and a pointer to a location on the FTP server where all the experimental result files can be located. Similar to the files for the models and experiments, each simulation tool may produce different log files as a result of running an experiment. Regardless of what the specific files are, they should all be placed in the location pointed to by the experimental results entity. In addition a description document (.doc) containing any comments related to the performance or the results of the experiment should also be placed in that location. As an example, the $\mathrm{CD}++$ Builder toolkit generates a $\log$ file $(. \log )$ and an (.out) file as a result of running an experiment.

\subsection{Summary}

This chapter introduced the architecture for a repository of models and experiments. The entities outlined in this chapter form the heart of the repository and provide the mechanism that allows the efficient storage, searching and retrieval of any item stored in the repository. The use of a relational table to store these entities makes it easy to query the database using the descriptive, human friendly, information for each entity while at the same time the ability to link tables together enables the repository to maintain the essential relations between these entities. These relationships can be used to make easier a user's search. For example, a user may want to retrieve all experiments related to engine models. Since a query on "engine" can easily return the list of engine models and since all models have links to their experiments a list of the experiments can be easily 
constructed and displayed to the user. The same applies for any other relationship between the stored entities.

By placing the storage engine on a computer connected to the internet, this architecture allows any user to connect to, search, download, and upload items to the database. This promotes the idea of creating an 'open source' repository of DEVS models where users across the world can share and improve upon models and experiments.

The flexibility of this architecture is in that it can be used to create a repository specific to any DEVS Simulation toolkit. In fact, if at some time in the future a standard file format to represent DEVS models were agreed upon by all DEVS simulation tools, this repository architecture would be perfectly suited to store these standard format files and thus would provide a platform for interoperability between different DEVS Simulation tools.

In the next chapters, an implementation of this architecture for the $\mathrm{CD}++$ Builder Toolkit is presented as a prototype application called the $\mathrm{CD}++$ Repository. 


\section{Chapter 5: The CD++ Repository - Software Architecture}

The $\mathrm{CD}++$ Repository introduced in this chapter is an implementation of the architecture presented in the previous chapter. It is an application designed to work as a part of the $\mathrm{CD}++$ Builder toolkit [4]. The $\mathrm{CD}++$ Repository runs as a stand-alone application that interacts with the $\mathrm{CD}++$ Builder by downloading models and experiments to $\mathrm{CD}++$ Builder project folders within Eclipse. The CD++ Repository provides the facilities to connect to, access, add to, and manipulate a central database of DEVS models and their experimental frames. The CD++ Repository uses a MySQL Database as its DBMS. This chapter describes the software architecture and design of the CD++ Repository Software.

\subsection{Overview of the CD++ Repository Software Architecture}

The $\mathrm{CD}++$ Repository has a client-server architecture with the client application being the $\mathrm{CD}++$ Repository portion of the $\mathrm{CD}++$ Builder plug-in, and the server being the database server running on a remote machine where all of the data objects are stored. The CD++ Repository is a "thick client" application in the sense that all of the business logic is carried out on the client machine while the interaction with the server is solely to send and retrieve data to and from the database.

The CD++ Repository's client application is designed following a layered architecture. 
Typically a layered architecture has three layers, a presentation layer at the top underneath it the business layer and finally the persistence (or database access) layer. The $\mathrm{CD}++$ Repository's client application is made up of two layers, the presentation and the persistence layer. Data is exchanged directly between the persistence layer and the presentation layer using Business Objects and any required business processing is done directly in the presentation layer objects or by the business objects. As the names imply the presentation layer is concerned with interactions with the user through displaying data retrieved from the database and collecting data to send to the database. The presentation layer uses the persistence layer to search the database, retrieve data from the database, make decisions about what to display, send data to the database, and detect conflicts with the database. One final important part of the architecture are the Business Objects. These objects encapsulate the business data and their behaviours. The Business Objects are used to exchange data between the presentation and persistence layers and their behaviours are used by the presentation layer to perform some processing on the data in the objects.

Figure 8 below gives a high level view of the CD++ Repository's client application. As can be seen in the diagram, a major part of the persistence layer is the Hibernate Java Package. This is the object relational mapping tool used by the $\mathrm{CD}++$ repository to map the Business Objects to their corresponding Relational Tables in the database. More details about Hibernate are presented later in this Chapter. Also from the diagram, one can see that in the presentation layer the CD++ Repository's Gui package makes use of 
the BIRT (Business Intelligence reporting Tools) package. BIRT is an Eclipse plug-in that enables the design and the run-time generation of business reports and it is used in the display of search results to the user. Using BIRT gives the search results page a professional look while at the same time making it easily modifiable and maintainable. More details about BIRT are presented later on in this chapter. 


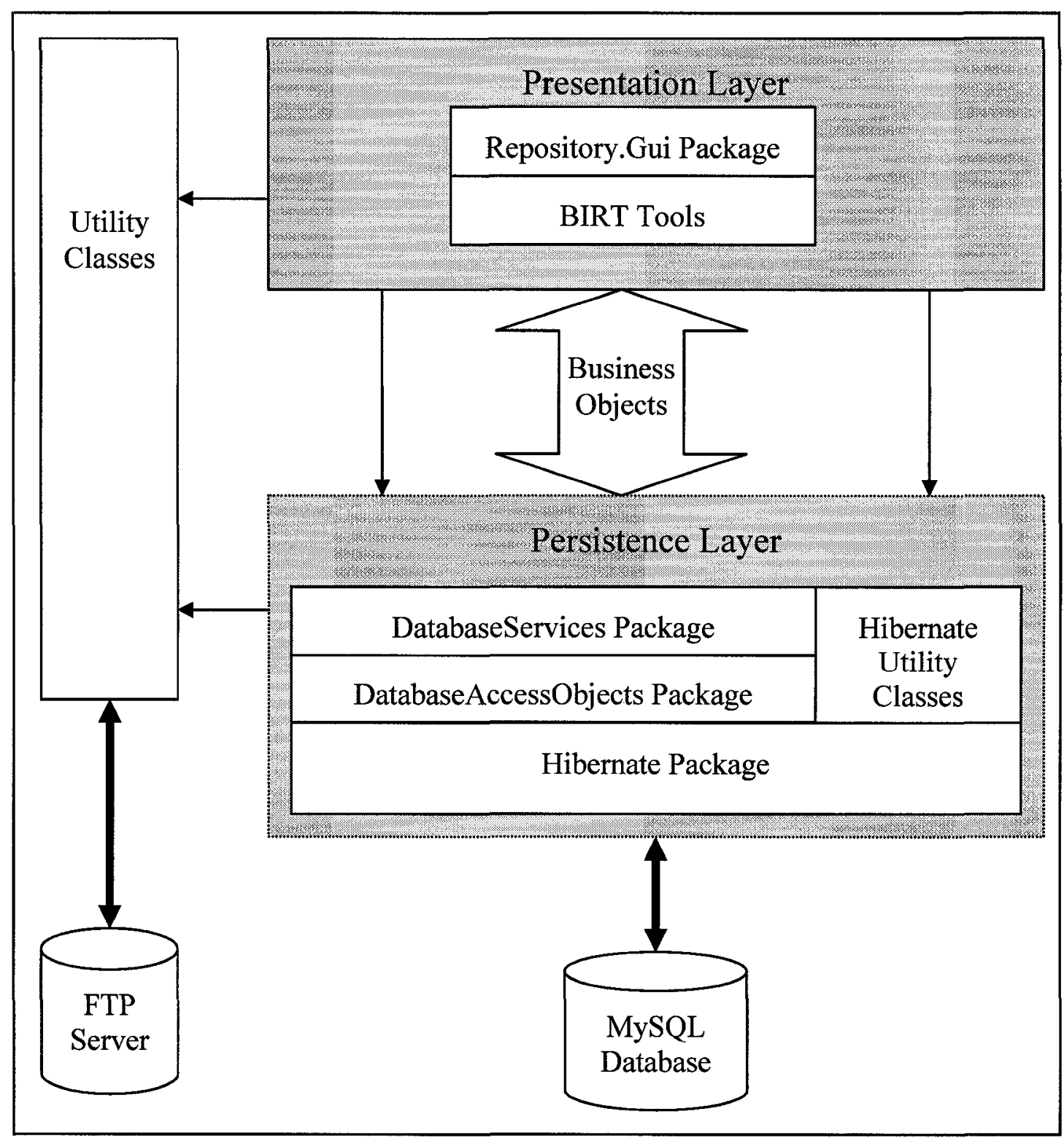

Figure 8: CD++ Repository Software Architecture

The next few sections will describe in more details the software design of the CD++ Repository. First, the Business Objects around which the whole software revolves are described. Then the mapping of those java Objects into Relational Database tables via the 
use of Hibernate is discussed. Following that, the database services that were built to access the database are introduced. Finally, the User Interface packages are discussed along with how BIRT is used.

\subsection{The CD++ Repository Business Objects}

The $\mathrm{CD}++$ Repository Business Objects (BOs) are the java objects that represent the entities that the $\mathrm{CD}++$ Repository software is designed to support, and are thus the objects of most importance to the design. The class diagram shown in Figure 9 below illustrates the most important BO Java classes. For simplicity this diagram does not show the classes representing the Experimental Frame's "other information" (i.e. the inputs and outputs for the experimental frame and the ranges of these input and outputs). 


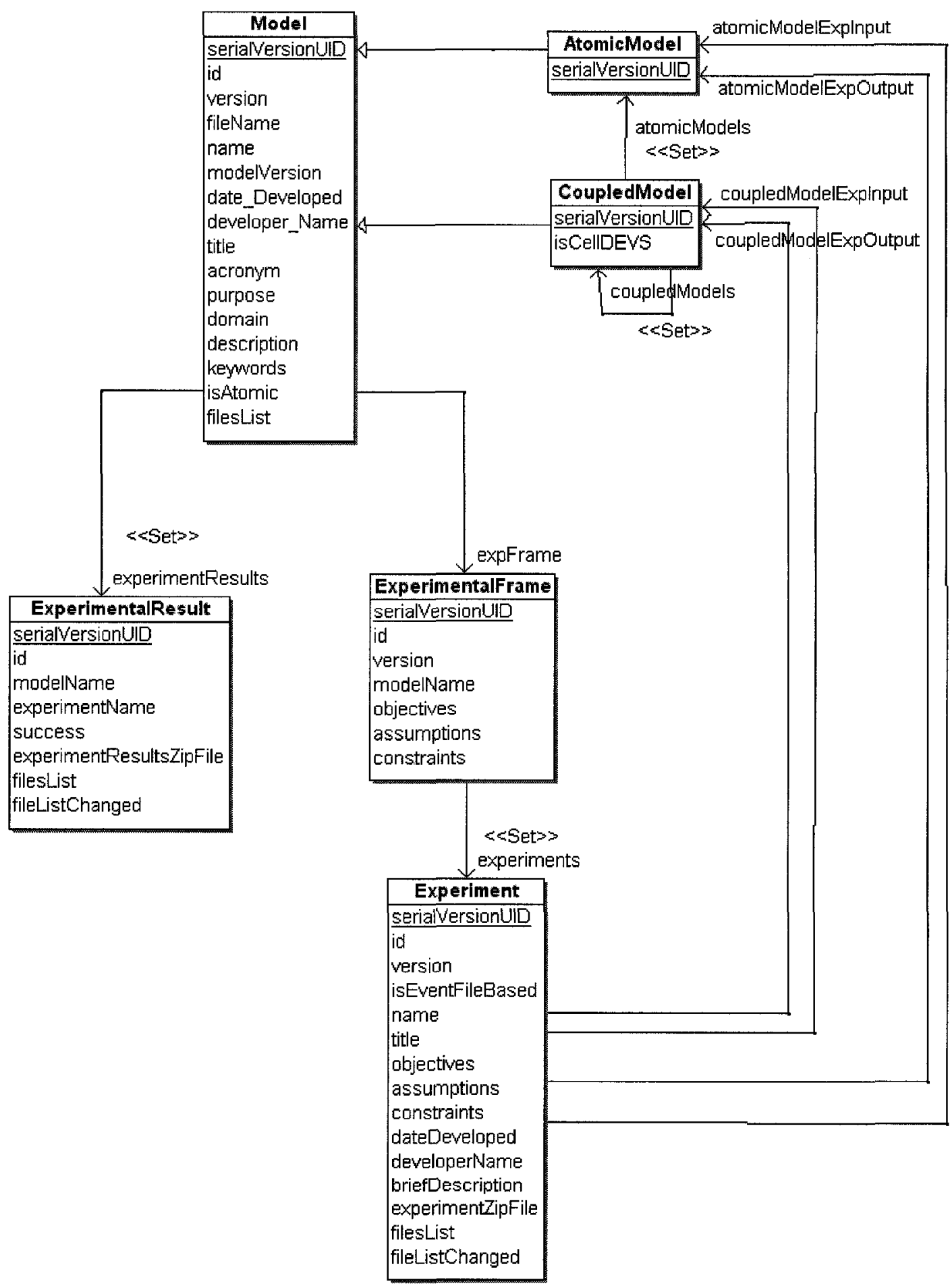




\section{Figure 9: CD++ Repository Business Objects Class Diagram}

As the class diagram shows, the central class is the Model class. The attributes and methods of the Model class represent all of the information that relates to both Atomic and Coupled models. In addition, the Model class contains a set of ExperimentalResults objects and an ExperimentalFrame object. This reflects the fact that each model can have many Experimental Results and only one Experimental Frame. Furthermore, the ExperementalFrame class contains a set of Experiment objects representing the experiments that can belong to a given model. The AtomicModel class and CoupledModel class both extend the base Model class and thus inherit all of its methods and attributes. While the AtomicModel class is not much different from the base Model class, the CoupledModel class has as attributes a set of AtomicModel objects and a set of CoupledModel objects representing the fact that coupled models have child models that can be either coupled or atomic or both. Finally, the Experiment class has two AtomicModel class objects and two CoupledModel class Objects. These represent the possibility that an Experiment can be model-based (as opposed to event-based) in which case it would have a model as a 'generator' and another model as a 'transducer' and these models can be either coupled or atomic models.

A quick inspection of the attributes of the Model class reveals that most of the attributes are used to hold the Model Data information that is entered by the user when a model is uploaded to the repository. Similarly, the attributes of the ExperimentalFrame, 
Experiment, and ExperimentalResult classes hold the ExperimentalFrame Data, the Experiment Data and the ExperimentalResult Data respectively. In addition, the Model, Experiment and ExperimentalResult classes each have an attribute that holds the name of a (.zip) file. This is the zip file stored on the FTP server and containing the relevant files for each object. The information contained in these attributes is the information that is eventually stored in the database as data entries in the appropriate columns of the appropriate database tables. A few of the class attributes however are used only during the life of the object and are not persisted to the database.

For simplicity, the class diagram below does not show the methods for each class. In general, each class attribute has getter and setter methods. These methods must be named according to the Java Bean Specification guidelines so that a generic tool like Hibernate is able to recognize them. The naming convention is simple: for an attribute named ' $f o o$ ' the getter and setter methods are named 'getFoo O' and 'setFoo O' respectively. Some classes have other methods that perform simple operations related to each class. The

more important of these methods for each Class are described in the following subsections.

\subsubsection{Model Class Methods}

The following methods are defined for this class, and are inherited by both the 
CoupledModel and AtomicModel classes:

- getExpFilename () : Given the name of a particular experiment, this method finds the matching experiment in the set of experiments for the current model, and returns the name of the (.zip) file for that experiment.

- getMatchingExperiment(): Given the name of a particular experiment, this method finds the matching experiment in the set of experiments for the current model, and returns the experiment object.

- createZipFile(): This function creates a (.zip) file containing all of the files related to the current model and returns the name and path of the created (.zip) file.

\subsubsection{CoupledModel Class Methods}

The following methods are defined for this class:

- findCoupledSubModelByName(): Given a model name, this method uses recursion to find the coupled model in the tree of child models that possesses the passed in name. If it is found, the coupled model object is returned.

- findParentofCoupledSubModelByName(): Given a model name, this method uses recursion to find the coupled model in the tree of child models that possesses the passed in name. If found, the parent model of the found coupled model is returned. 
- retrieveAllAtomicSubModels(): This method returns a set of all of the Atomic model objects that exist in the tree of sub-models for this coupled model.

- retrieveAllCoupledSubModels(): This method returns a set of all of the Coupled model objects that exist in the tree of sub-models for this coupled model.

- sameStructure(): Given a coupled model Object, this method determines whether the structure of the passed in model matches the structure of this coupled model.

- findChildCoupledModelByName(): Given a model name this method finds the immediate child of this model that is a coupled model and that possesses the passed in model name. If found the coupled model object is returned.

- getAllFileNames(): This function returns a list of the names of all of the (.zip) files for all of the models in the models tree under this model.

- updateModelInfo(): Given a coupled model object, this method updates selected attributes of the current coupled model with the values from the passed in coupled models.

\subsubsection{Experiment Class Methods}

The following methods are defined for this class:

- genNextExpName(): This method cerates a unique name to be used for the next Experiment object to be created. 
- createZipFile(): This function creates a (.zip) file containing all of the files related to the current experiment and returns the name and path of the created (.zip) file.

\subsubsection{ExperimentalResults Class Methods}

The following methods are defined for this class:

- createZipFile(): This function creates a (.zip) file containing all of the files related to the current ExperimentalResults object and returns the name and path of the created (.zip) file.

\subsubsection{Methods for all Business Object Classes}

The equals () and hashCode( $)$ methods are methods defined by the java language for any java object. For all Business Objects in the $\mathrm{CD}++$ Repository these methods are overridden to provide proper object identity for the objects. For the most part the CD++ Repository identifies two objects as being equal if they both have matching name attributes. The ExperimentalResults class is an exception; it uses the names of the model and experiment to which it refers to provide object identity. Overriding these methods to properly define object identity is necessary for using Java Collections and for Hibernate to handle collections properly. 


\subsection{Hibernate and Object-Relational Mapping}

The central question to a database application like the $\mathrm{CD}++$ Repository is how to manage the application's persistent data. Persistent data is the data that needs to be stored to disk so that it can be retrieved later for further processing. For the CD++ Repository, the persistent data for the Model, Experimental Frame, Experiment and Experimental Results were all presented in Chapter 4 of this work. The Business Objects described in the previous section are the objects that are used to hold this persistent data during the life of client session. The persistent data is stored on disk in the application's database residing on the database server. As mentioned earlier, the database used for the $\mathrm{CD}++$ Repository is the MySQL database, which is a Relational database that stores the persistent data in tables.

The problem that arises is that while the java application uses an object-oriented representation of the business entities (the Business Objects), the relational database uses a tabular representation of the same business entities (the database tables). The relational model and object-oriented model are two fundamentally different models of data representation [30]. This problem has been re-searched thoroughly and there are numerous attempts at solutions to it in the industry. One of these solutions is Hibernate, which is the solution used in the implementation of the $\mathrm{CD}++$ Repository. Hibernate uses Object/Relational Mapping (ORM) techniques to solve the object / relational mismatch [30]. 


\subsubsection{Introduction to Hibernate}

Hibernate is an open source Java package that uses ORM to solve the object / relational mismatch problem inherent in java applications that implement data persistence by storing persistent data in a relational database using SQL. Hibernate makes it seem like the java objects themselves are being saved and retrieved from the database without the programmer having to worry about how the data is being stored and retrieved from the tables in the database. This allows the programmer to concentrate more on the business problem and worry less about writing SQL to access the database. Using an ORM solution provides the following advantages [30]:

1. Productivity: Hibernate eliminates the need to worry about accessing the database, leaving the developer free to concentrate on the business problems and thus reducing development time.

2. Maintainability: Hibernate reduces the number of lines of code needed, thus making the code more easily maintainable.

3. Performance: Hibernate provides great performance given the amount of time saved by not having to implement a hand-coded persistence solution. It could be argued that the performance of a hand-coded persistence solution will always be at least as good as an automated one like Hibernate, however the amount of effort needed eclipses any such claimed performance benefits. 
4. Vendor Independence: Hibernate buffers the java application from the underlying SQL database and SQL dialect. As a result changing the underlying database becomes easier when an ORM solution like Hibernate is used.

A high-level view of the architecture of Hibernate as used in the CD++ Repository is shown in the Figure 10 below:

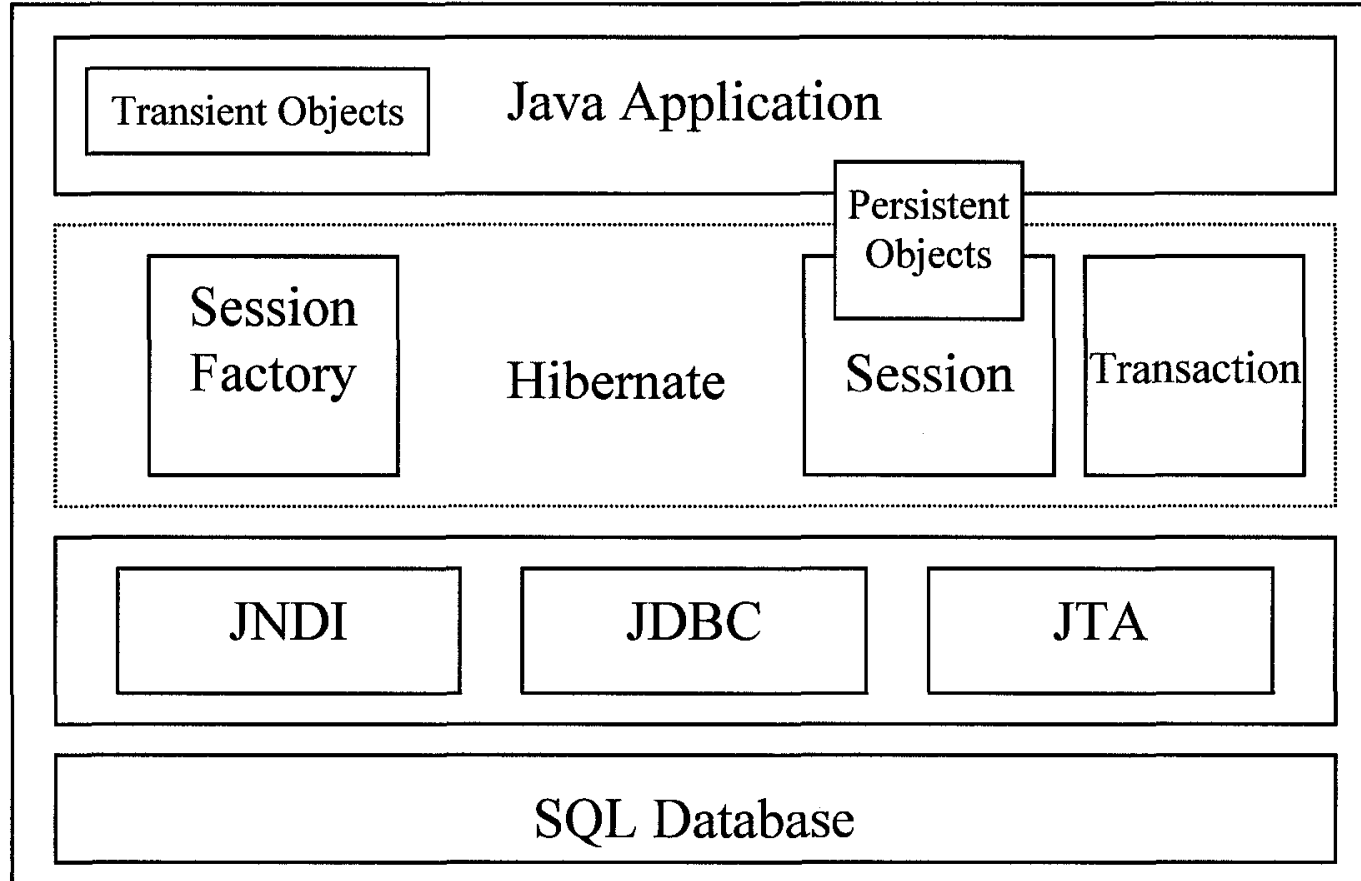

Figure 10: A High Level View of the Hibernate Architecture

As shown in Figure 10, Hibernate sits between the database and the java application. It controls the connection to the database and thus controls the issuing of SQL statements to the database. The way Hibernate interacts with the underlying database is beyond the scope of this discussion. It is important however to explain the interface between Hibernate and the Java application. The following are the core Hibernate interfaces of 
interest to the reader:

1. Configuration Interface: This object is the first object created when using Hibernate, and as its name implies it is used to configure Hibernate. This object can be used to configure the Hibernate properties and mapping-documents either manually or through an XML configuration file (hibernate.cfg.xml). This object is used to create the Session Factory.

2. Session Factory: The Session Factory object is thread-safe and is not lightweight; an application that connects to a single database is intended to have only one session factory shared among all of its application threads. The session factory uses the configuration loaded into the configuration object to initialize Hibernate. Among other things, the configuration tells Hibernate the IP address, port, username, and password of the SQL server. The application uses the session factory to obtain Hibernate sessions.

3. Session Interface: The Session Interface is the main interface between applications and Hibernate. An instance of this object is not thread-safe, is lightweight, and is intended to be created and destroyed frequently, perhaps for every database request. A session can be seen as a persistence manager. Objects loaded in a session are tracked by hibernate and any changes to them (from what is in the database) is detected and when the transaction ends the changes are committed to the database. Therefore, whenever an application manipulates objects and wants the changes to be reflected in the database it should do it within 
a session. Objects loaded in a session are called "Persistent Objects" in Hibernate terminology; this is as opposed to "Transient Objects" which are objects that have a representation in the database but are not currently loaded in a session, and "Detached Objects" which are objects that were loaded in a session but were either evicted from the session or the session itself was closed.

4. Transaction Interface: The Transaction Interface places an abstraction level between the application code and the underlying database transaction implementation. This allows the application to control its transaction boundaries through a consistent Hibernate object, rather than depend on underlying implementations (e.g. JDBC Transaction or JTA Transaction).

Having introduced Hibernate's basic architecture and interfaces, there is one important item left to complete the picture, and that is how Hibernate is able to map the java objects into SQL database tables. The answer is by using XML files called Hibernate mapping files (.hbm.xml). Each class that needs to be persistent must have a Hibernate mapping file that defines the database table that the class maps to and that maps the properties of the class to the appropriate columns or tables in the database. The Hibernate mapping file also defines other more advanced properties of the mapping including mapping of associations, cascading, and automatic versioning. The paths to all of the Hibernate mapping files are included in the configuration information for Hibernate. When the session factory is created, it loads those files and performs the mappings. The Hibernate 
mapping files are described in more detail in the next section.

\subsubsection{Mapping CD++ Repository Business Objects to Database Tables}

In Section 5.2 the main CD++ Repository Business Objects were introduced. Those business objects hold all of the information that needs to be persistent in the application, and thus are the objects that have to be mapped to database tables using the Hibernate mapping files. Figure 11 illustrates how the mapping of a $\mathrm{BO}$ is done; it shows the AtomicModel class's mapping file.

At the top of the mapping file the first thing defined is the name of the class being mapped and the name of the table to which it is mapped. The id and version attributes of the class are Hibernate specific; they have been added to the class just for Hibernate. The id maps to the Primary key of the objects in the database table and is used by Hibernate for object identity issues, and the version is used by Hibernate to do automatic versioning, and thus facilitate concurrency control. After these two items is the actual persistent information that this class holds. These all map directly to columns in the database table, except the last two: the Experimental Frame and the Experimental Results. The association between an Atomic Model and its Experimental Frame is a oneto-one foreign key association. In Hibernate, the foreign key association is represented using a <many-to-one $>$ mapping element with the "unique" constraint set to true. This adds a column with the title "Exp_Frame_ID" to the Atomic_Model table. 
$<$ ?xml version=" 1.0 " encoding="UTF-8"? $>$

$<$ !DOCTYPE hibernate-mapping PUBLIC

"-//Hibernate/Hibernate Mapping DTD 3.0//EN"

"http:/hibernate.sourceforge.net/hibernate-mapping-3.0.dtd">

$<$ hibernate-mapping>

$<$ class name $=$ "CDBuilder.repository.businessObjects.AtomicModel"

table="Atomic_Models">

$<$ id name="Id" column="ID">

$<$ generator class $="$ native" $>>$

$</$ id $>$

$<$ version name $=$ "version" column $=$ "Version" $>>$

$<$ property name $=$ "fileName" column="File_Name" $>>$

$<$ property name="name" column="Model_Name"/>

$<$ property name $="$ modelVersion" column $="$ Model_Version"/>

$<$ property name $=$ "date_Developed" column $=$ "Date_Developed" $>>$

$<$ property name $="$ developer_Name" column $=$ "Developer_Name"/>

$<$ property name $="$ title" column $="$ Title" $>$

$<$ property name $=$ "acronym" column $=$ "Acronym"/>

$<$ property name $=$ "purpose" column $="$ Purpose" $/>$

$<$ property name $=$ "domain" column="Domain_Area" $/>$

$<$ property name $=$ "keywords" column="Keywords"/>

$<$ many-to-one name="expFrame"

class="CDBuilder.repository.businessObjects.ExperimentalFrame"

column="Exp_Frame_ID"

cascade $=$ "all"

unique $=$ "true" $>$

$<$ set name="experimentResults"

table="Atomic_ExpResults_Map"

cascade $=$ "all" $>$

$<$ key column="Atomic_ID" $/>$

$<$ many-to-many

class $="$ "CDBuilder.repository.businessObjects. ExperimentalResult"

column="ExpResult_ID"

$</$ set $>$

unique $="$ true"/>

$<$ class $>$ 
$</$ hibernate-mapping $>$

Figure 11: Sample Hibernate Mapping File

The association between an Atomic Model and its Experimental Results is a one-tomany association. In Hibernate, the one-to-many association is best represented using a $<$ many-to-many $>$ mapping element with the "unique" constraint set to true. This creates a join table called Atomic_ExpResults_Map to join the Atomic_Model table with the Experimental_Results table with a unique constraint on the Expresult_ID column to force its multiplicity to one-to-many.

The mapping files written for the $\mathrm{CD}++$ Repository can be used by Hibernate to automatically generate the database tables. This is done by setting a property in the configuration file. Figure 12 below shows the database tables created for the $\mathrm{CD}++$ Repository. For the most part the database tables have similar names to the business objects to which they map. The Atomic_Models, Coulped_Models, Experiments, ExperimentalFrame, and ExperimentalResults database tables all map to the business objects that share their name. This covers all of the business objects described previously. The rest of the database tables in Figure 12 are join tables that represent the interconnections between the objects. The Coupled_Atomic_Map and Coupled_Coupled_Map tables represent the fact that a Coupled model can have Atomic and Coupled models as children. The Coupled_ExpResults_Map and Atomic_ExpResults_Map tables represent the fact that each atomic and coupled model 
can be associated to multiple experimental results. EF_Experiments_Map represents the fact that an EF can be associated with multiple experiments.

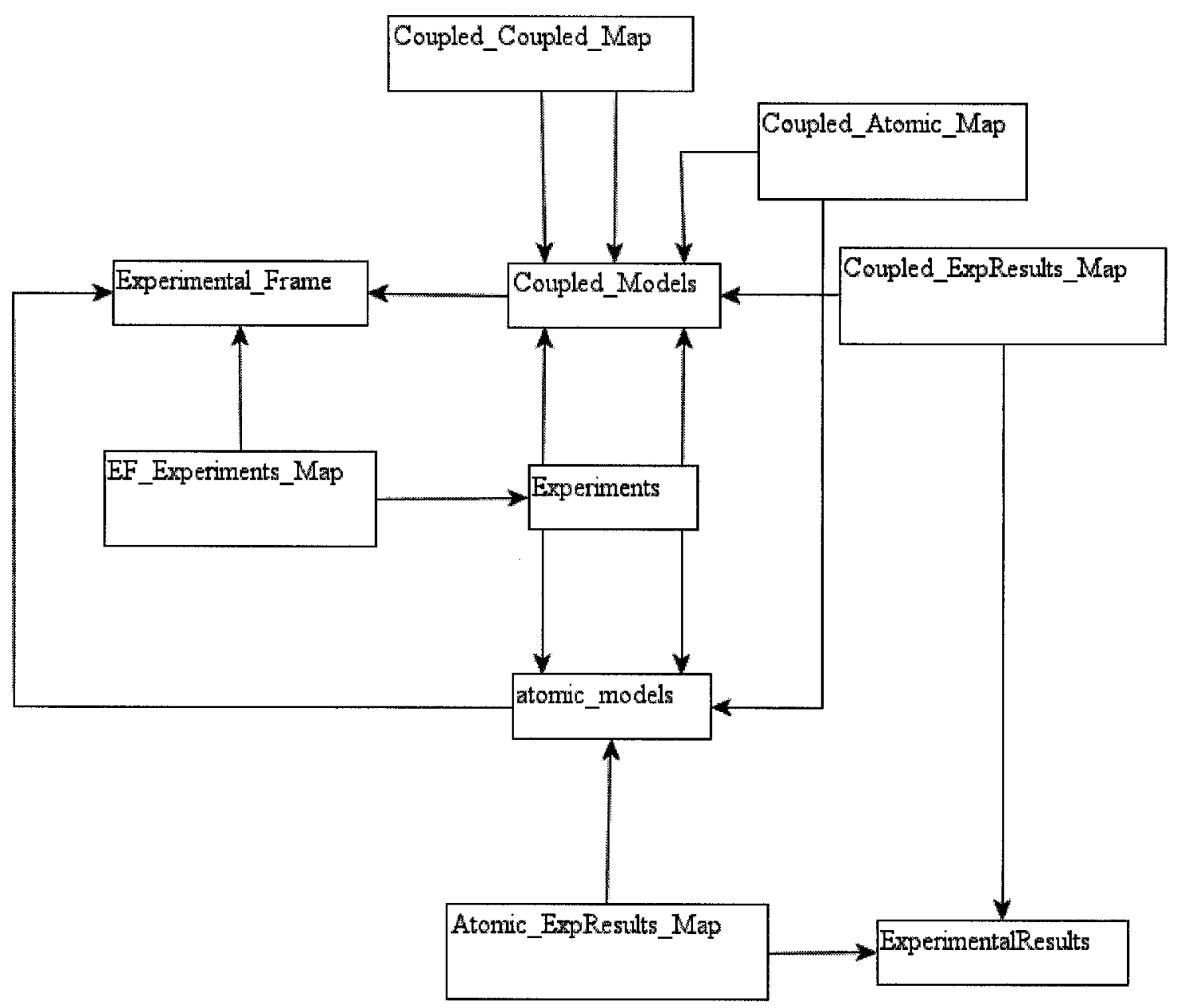

Figure 12: CD++ Repository Relational Database Tables.

\subsection{More Software Details}

The Java packages that make up the $\mathrm{CD}++$ Repository software can be divided into two 
main parts, persistence layer packages and presentation layer packages. In addition to these packages, the software contains a few supporting utility classes and the software also uses the BIRT (Business Intelligence Reporting Tool [31]) Eclipse plug-in to produce the reports presented to the user. The interested reader can learn more about these items in Appendix A. 


\section{Chapter 6: CD ++ Repository -The Client Application}

The $\mathrm{CD}++$ Repository aims to enable the users of $\mathrm{CD}++$ Builder to collaborate in their modeling work thus enabling the reuse of DEVS Models and their Experiments. The following sections describe the $\mathrm{CD}++$ Repository Client application's features that provide the users with an easy to use interface and enable them to achieve the ultimate goal of collaboration through model and experiment reuse.

\subsection{Uploading Models to the Repository}

When a user has developed a new model and is ready to upload it to the CD++ Repository, they are expected to have two main items ready, first the files(s) for their model and any sub-models, and second the Model Data and Experimental Frame Data for their model(s). The CD++ Repository prompts for this information. To simplify the uploading process and automate it as much as possible the user is initially only asked to enter the path of the model definition (.ma) file for the model to be uploaded. Using the (.ma) file alone, the Repository Software will automatically:

1- Detect what kind of Model this is. (Atomic, Coupled, or Cell-DEVS model?)

2- For Coupled models: Detect all of its sub-models and construct the full hierarchy of models under the parent model.

3- Establish a name for this model and all sub-models (if they exist).

4- Detect conflicts with models that already exist in the repository, and handle any 
conflicts appropriately.

5- For Coupled models: Detect whether any of the child models already exists in the Repository, and if they do handle the conflicts appropriately. Special checking of coupled-sub models to ensure they actually match the repository models.

6- For Coupled models: Construct a list of all the models that do not already exist and for which data needs to be collected from the user.

7- Finally, collect the required Model Data, Experimental Frame Data, and the files for the model and all if its child models (if applicable) and upload all of this information to the $\mathrm{CD}++$ Repository. (Note that the $\mathrm{CD}++$ Repository also provides the opportunity to collect Experiment(s) and Experimental Result(s) at this stage in the upload process; the next section will discuss the Experiment and Experimental Results uploads.)

The process by which each of these items is done is explained in the following sub sections, but an introduction to the structure of the (.ma) files is first needed to clarify the kinds of information that it contains about a particular model.

\subsubsection{The Model Definition (.ma) File Structure}

The CD++ Repository Tool parses the model definition (.ma) file of the model to be uploaded. All models in CD++ have [top] as the highest level model in a hierarchy of 
models [32]. In that sense even Atomic Models are presented in $\mathrm{CD}++$ as pseudo Coupled Models with [top] as the highest model with just the Atomic Model underneath. Under the [top] model in the (.ma) file, the components label is used to indicate the start of the listing of the components under the [top] model. If a component is an Atomic component, its name is presented like so "fool@foo" where fool is the name of the instance of the atomic model and foo is the name of the Class of the atomic model. On the other hand, if a component is a Coupled component then a name is presented for it in the components list, and then later in the file that name appears again between square brackets followed by another components label and a list of its own components. This continues until all components in the hierarchy are listed. In Addition, a type label is used to indicate if a coupled model is a Cell-DEVS model or a regular Coupled Model. In case it is a Cell-DEVS model, then no components are listed under it. Figure 13 below shows a sample (.ma) file of an atomic model on the left and another sample of a coupled model on the right. 


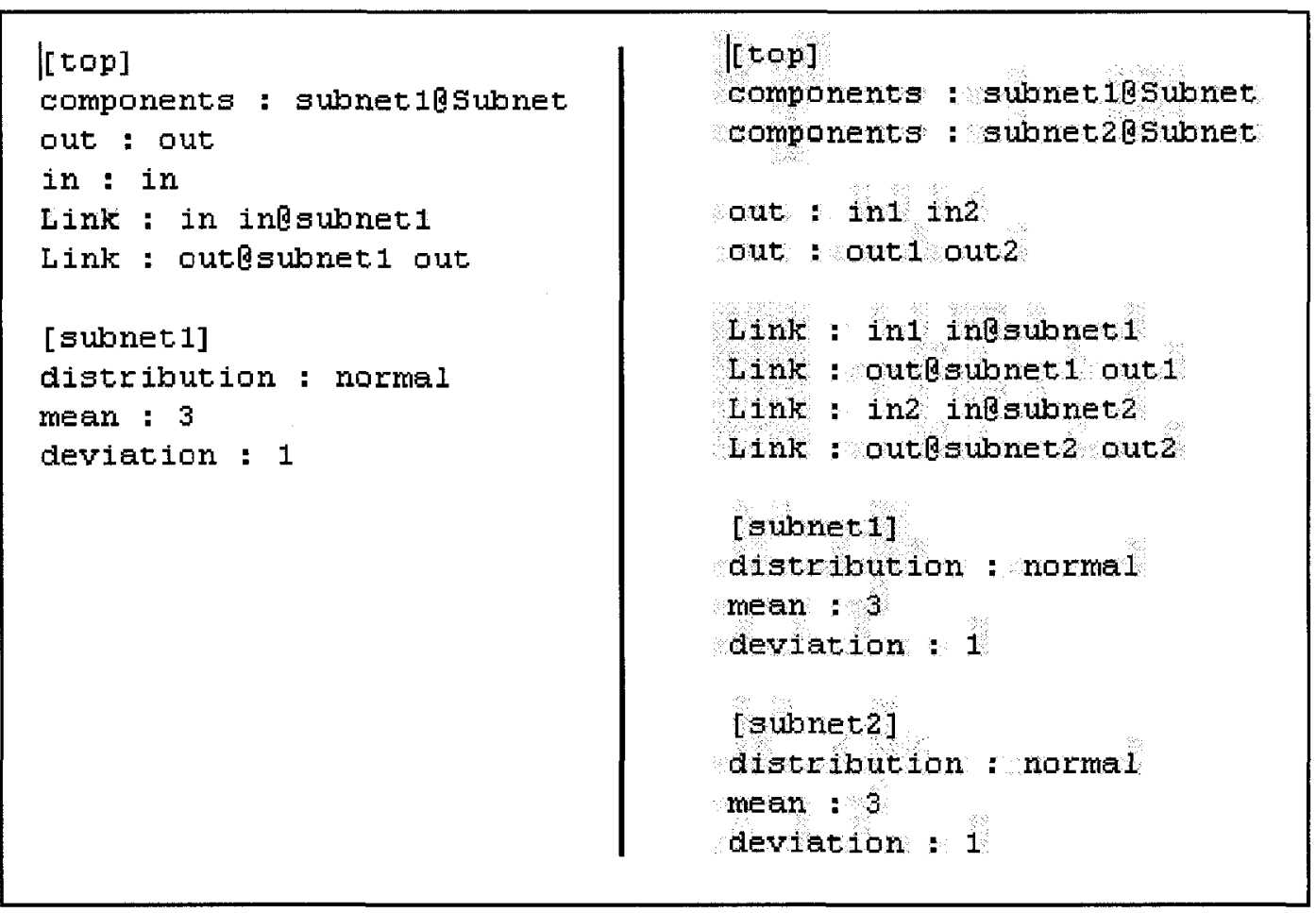

Figure 13: Sample Atomic and Coupled (.ma) Files

\subsubsection{Automatic Detection of Model Kind, Structure, Name and Conflicts}

When the model definition file (.ma) is parsed, the first thing to be determined is whether the Model being uploaded is Atomic, Coupled, or Cell-DEVS. It is clear that an Atomic Model's (.ma) file will only have one component defined in it, and that component will have an '@' sign separating the instance name from the class name in its identifier. Coupled Models on the other hand will have more than one component defined in the (.ma) file. Finally, Cell-DEVS Models can be distinguished by the tag cell after the type label. In the case of Coupled Models, the hierarchy of the child models can be easily 
constructed from the information in the file.

Having distinguished the kind of Model that the given (.ma) file represents and, in the case of Coupled Models, the model hierarchy the next step is to determine the name for these models. This model name is a key piece of information for the CD++ Repository since it is used as the unique identifier of a given model (i.e. no two models in the repository can have the same name). For Atomic Models the name of the Model is taken from the Class name (the portion after the '@' sign in the identifier). This was chosen for the following simple reasons:

- It is the identifier used for the Atomic Component in the (.ma) file and so it is recognizable by the user

- Being a Class name, it will usually be descriptive of the model in question.

- It is unique within the given model.

- It is the best available choice given the information available in the (.ma) file

For Coupled (and Cell-DEVS) models, there is no name within the (.ma) file that would serve the same purpose as the Class name does for Atomic models. For example, a Coupled model made up of two Atomic Models will have no mention of the Coupled Model's name itself in the (.ma) file. As a result, it was decided to take the name of the (.ma) file itself as the best candidate for the name of the Coupled Model. Note that there is a convention of adding the letters ' $M A$ ' at the end of (.ma) filenames, so if the 'MA' 
exists it is discarded before taking the filename as the name of the Coupled model. One final note about model names; using the above mentioned naming method the names of all Atomic sub-models in a given (.ma) file can be determined simply by parsing the file, however for all Coupled sub-models the user has to be prompted for the (.ma) file of the given sub-models so that the names can be derived from the (.ma) filenames.

Having derived the names of the models a check is performed to ensure that the models do not already exist in the CD++ Repository. As mentioned earlier the name of a model is required to be a unique identifier of the model and no two models in the repository can have the same name. For atomic models, the check is easy; if the model's name already exists in the repository, a message is presented to the user informing them of the conflict and advising them to re-name their model and try to upload it again if they are sure that it is different from the one already in the Repository.

For Coupled Models the task becomes a bit more complex when we consider all the child models. Similar to the Atomic Model, the Coupled Model's name itself can be easily checked to verify that it does not already exist in the repository. If it already exists a message is presented to the user informing them of the conflict and advising them to rename their model and trying to upload it again if they are sure that it is different from the one already in the Repository. However the same cannot be done if the Coupled Model's name itself is unique, but one or more of its child models is not unique (i.e. already exists 
in the repository). The reason the upload cannot be rejected in this case is that there are legitimate cases where the child models can already exist in the repository. For example, suppose that Atomic Models Engine and Wheels already exist in the CD++ Repository and a model designer downloaded them and used them to create a new Coupled model called Car. When then designer uploads the Car model to the repository it is expected that the child models Engine and Wheels will be found to already match models found in the Repository. Therefore, in this case the CD++ Repository will present a warning to the user that the sub-models Engine and Wheels have been found to already exist in the repository and that they will not be uploaded again. The user is informed that the Car model will be linked to the existing Engine and Wheels models and is advised to cancel the upload operation if they are not sure that these are the same models used in his/her Car model.

Another final check is performed by the system to ensure no corruption of data occurs in the Repository. Suppose a top coupled model $A$ contains a child coupled model $B$ which in turn contains two sub-models $X$ and $Y$. Suppose also that the repository already contains a coupled model $B$. When the model $A$ is uploaded to the repository, the system determines that model $B$ already exists, but does not stop here and generate the aforementioned warning to the user. It does a further check to ensure that the coupled model $B$ in the repository is in fact the same as the coupled model $B$ in the uploaded model. This check is done by comparing the sub-models of the two $B$ models. If for 
example the repository's version of B had sub-models $R$ and $S$ instead of X and $Y$ the user is told of the conflict and the upload operation is aborted. If however the repository's version of B is the same as the one being uploaded then the aforementioned warning is generated, and the upload operation may proceed.

\subsubsection{Collection of User Entered Data}

The next stage in the upload process is to collect all of the data related to the model from the user. For Atomic Models this simply means collecting the data for the model being uploaded. For Coupled Models however this means collecting the data for the model being uploaded and all of the sub-models that have been determined to be new (i.e. do not already exist in the repository). The following discussion applies to each uploaded model, regardless of whether it is the main model being uploaded or a sub-model.

The user is presented with a window in which to enter the information about the model. The top part of the window enables the user to enter all of the Model Data, except for the Model Name because it is automatically generated as mentioned earlier. The middle part of the screen enables the user to enter the paths to the relevant files. For Atomic models, these are the paths to the model definition file (.ma), the model class files (.h) and (.cpp), and the path to the description document. For Coupled Models they are the paths to the model definition file (.ma) and the description document. Finally, the bottom part of the 
screen enables the user to enter the Experimental Frame Data and any Experiments related to the model. The following section explains the process of uploading an Experiment and the related Experimental Results. A screen shot of the top part of this window is shown in Figure 14; the bottom part of the window is show in Figure 15.

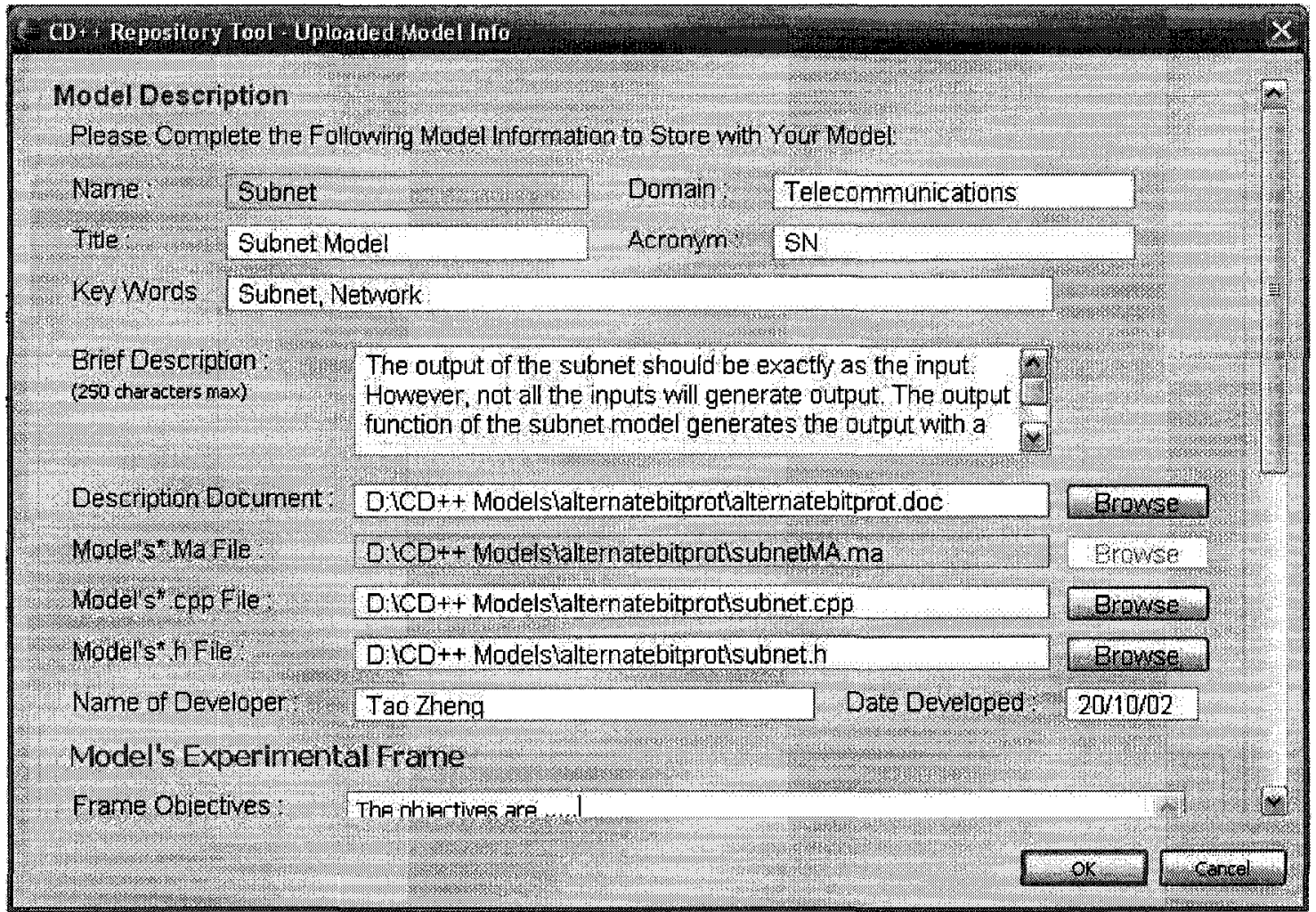

Figure 14: Atomic Model Upload Window Screenshot - Part1 


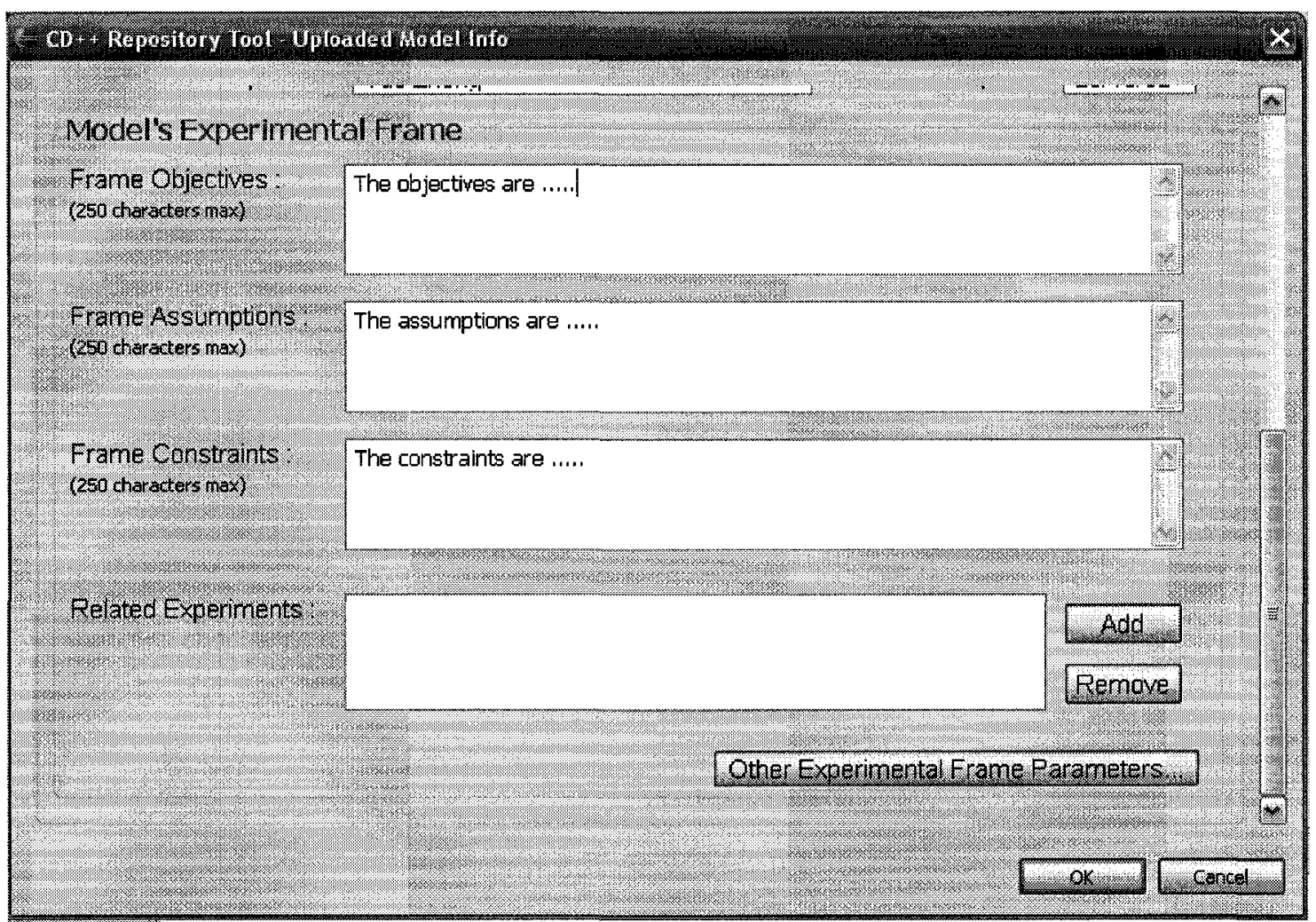

Figure 15: Atomic Model Upload Window Screenshot - Part2

\subsection{Uploading Experimental Frames, Experiments and Experimental Results}

Experiments are entities of their own that exist independently in the CD++ Repository and that may be linked to one or more models by adding them to the model's experimental frame. This means that a user can design the Model and its Experiments either at the same time or separately, and can upload them to the Repository also either in the same session or in separate sessions. Therefore, the CD++ Repository allows the user to enter a new Experiment into the Repository in the following two ways. The first way is 
for the user to select to upload an Experiment from the main menu. In this case the experiment is added to the repository but is not linked to any model. Linking this experiment to a model can be done later during upload of the model, or by editing an exiting model. The second way is for the user to add a new experiment to the repository during the upload of a new model. In this case, the Experiment will be linked to the newly uploaded model. It should be mentioned here that when adding an experiment to a model during upload of the model the user is given the choice of entering a new Experiment or linking the model to an existing Experiment. If adding an existing Experiment is chosen then the user is presented with a search window followed by a search results window from which the Experiment can be chosen. In this case, the following description about adding an Experiment does not apply.

Whether uploading a new experiment from the main menu or during the upload of a model, the user is required to specify the type of Experiment that is being uploaded; namely, an Event based Experiment, or a Model based Experiment. If the user selects an Event based Experiment, then they are presented with a window in which to enter the information about the experiment. The top part of the window enables the user to enter all of the Experiment Data. The bottom part of the window allows the user to enter the path to the detailed description document and the other experiment files (.ev, .bat, .val, .pal., .drw). This is shown in the screen shot in Figure 16 below. If a user selects a Model based Experiment on the other hand, they are presented with a similar window containing 
the same top part of to enter the Experiment Data. The bottom part of the window however gives the user the ability to enter the path to a description document for the experiment, and to add a model that will act as the input "generator" for the experiment and another model that will act as the output "analyzer" for the experiment. This is shown in Figure 17 below. The "generator" and "analyzer" models can be selected from models already existing in the repository or can be added as new models. If selected from exiting models in the Repository the search page is presented followed by the search results page from which the user can select the model of their choice. The next Section talks more about searching the repository and the search results page. 


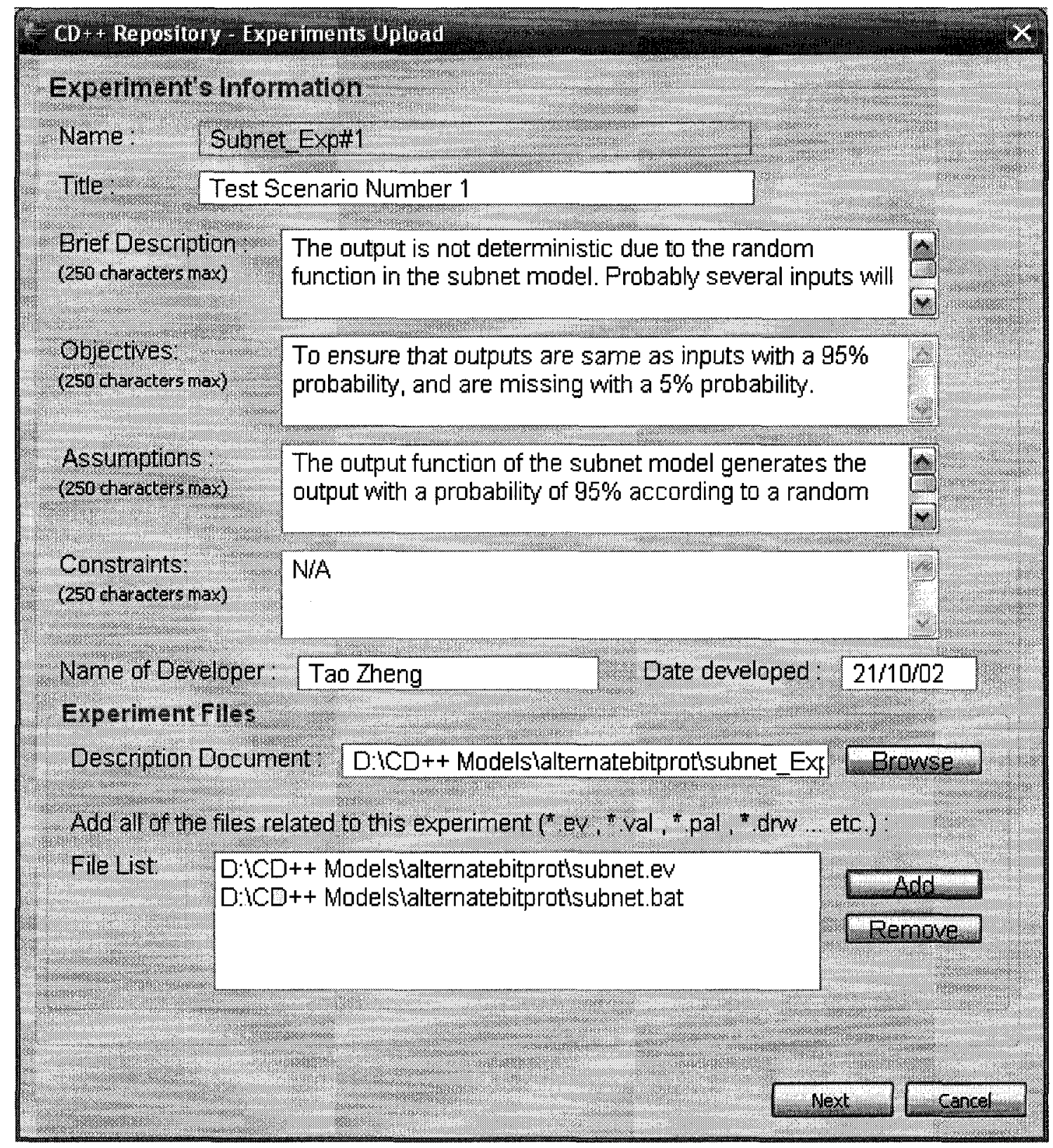

Figure 16: Event Based Experiment Upload Window Screenshot 


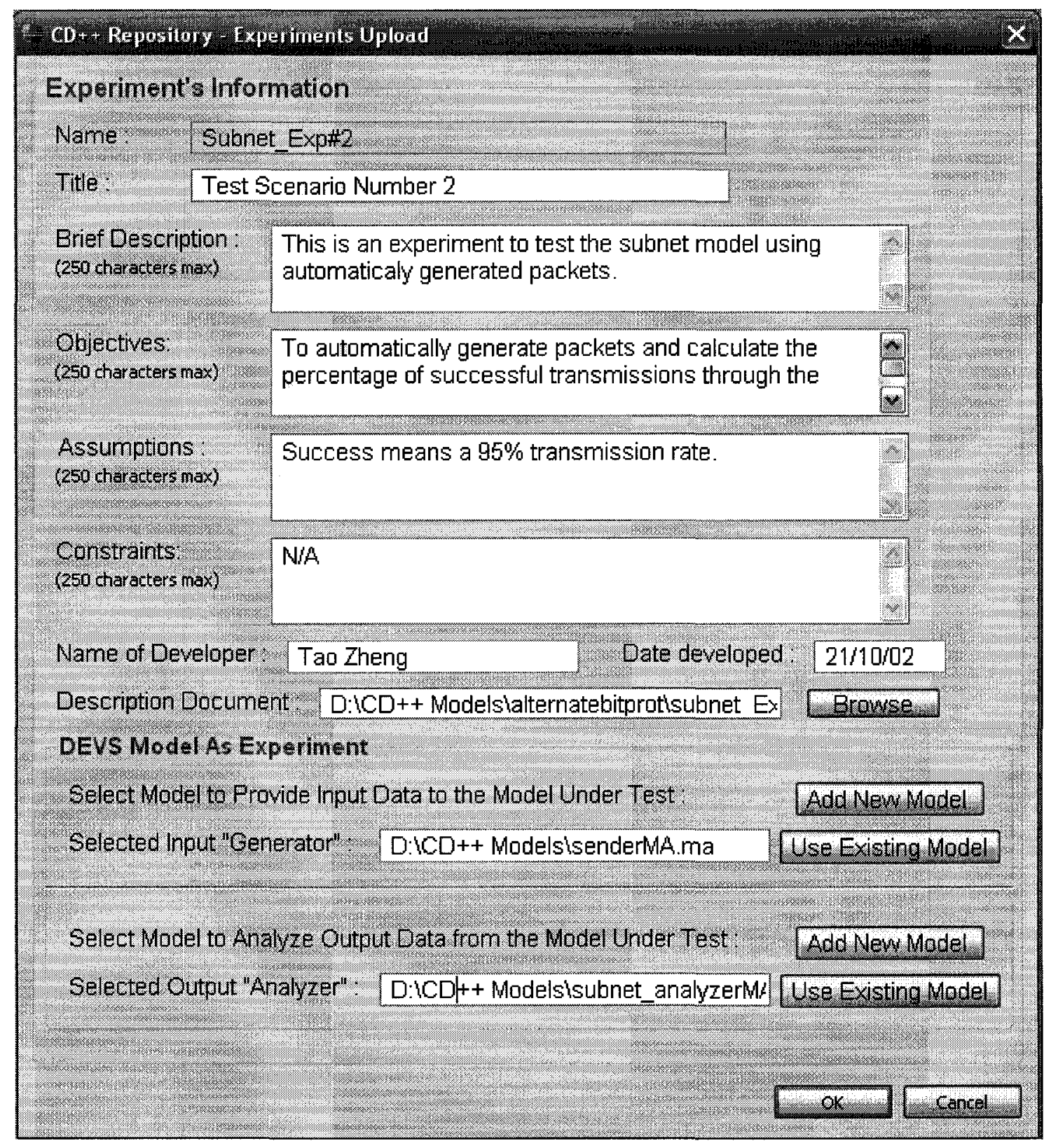

Figure 17: Model Based Experiment Upload Window Screenshot

The last item that needs to be uploaded is the experimental results for a given modelexperiment pair. The user is given the opportunity to enter the Experimental Results Data 
at the following two places in the application. The first is during the uploading of a new model to the Repository and right after an Experiment is added to the model, the user is given the opportunity to add Experimental Results data for the model-experiment pair. The second place is when an existing model is chosen for editing, the user can add the Experimental Results Information for any experiment within the model's Experimental Frame. A screenshot of the window used for adding the Experimental Results information is shown in Figure 18 below.

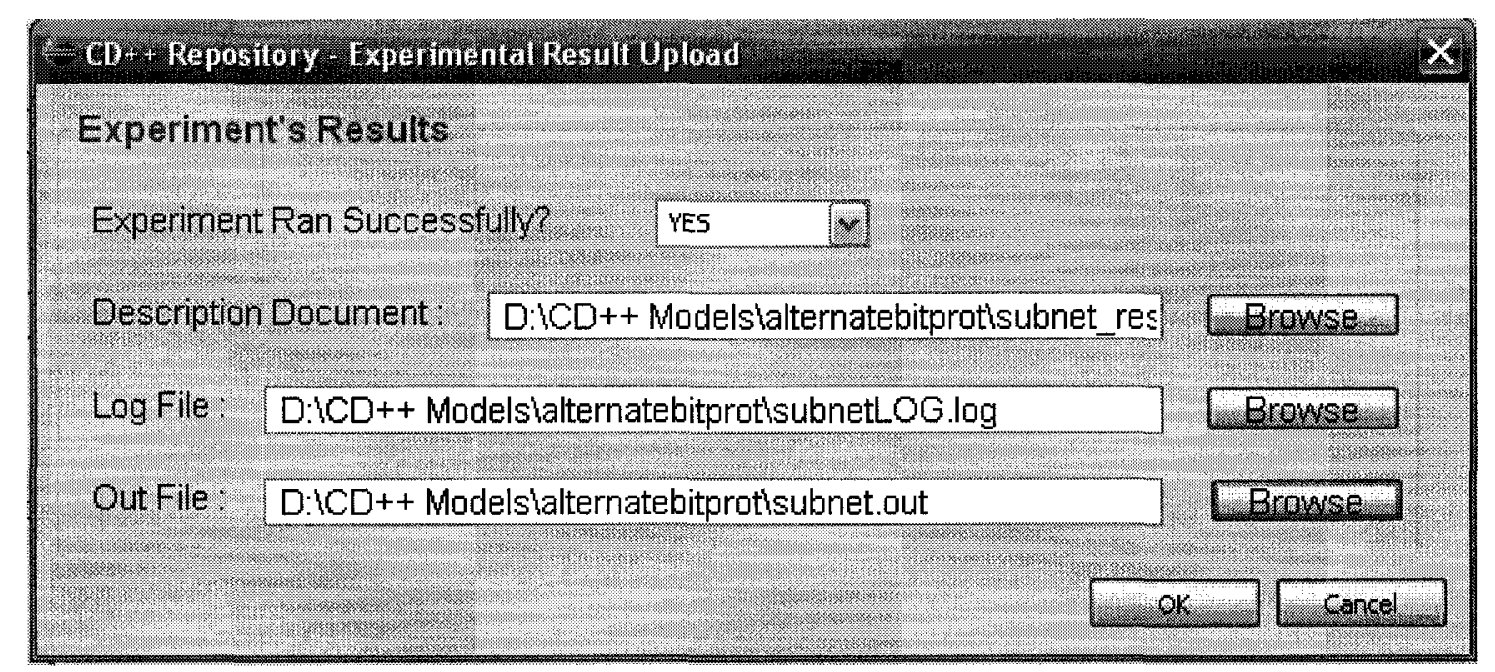

Figure 18: Experimental Results Upload Window Screenshot

\subsection{Searching and Downloading Models and their Experiments}

In the past few sections the discussion concentrated on the ability to upload Models, Experimental Frames, Experiments and Experimental Results to the CD++ Repository's 
database. This section and the next discuss the ability to search for Models and Experiments in the $\mathrm{CD}++$ Repository's database and download them to a $\mathrm{CD}++$ builder project. Again it is important to note that as far as the $\mathrm{CD}++$ Repository is concerned Models and Experiments are separate entities that may or may not be linked to each other; therefore the CD++ Repository allows the search and download of each of these entities independently of the other. When downloading a model however, the CD++ Repository does allow the option to download any attached experiments.

\subsubsection{Searching for and Downloading Models}

A user can select to download a model from the main menu of the CD++ Repository. When such a selection is made, the user is presented with a search dialogue window. This window allows the user to search for a model based on most of the Model Data described earlier in this chapter. A screenshot of this window is shown in Figure 19 below. This is a simple search window that has the following search features:

- The search criteria entered by the user does not have to be full words or sentences, for example a user can enter "engine" in the title criteria and the search engine will find all models with the word "engine" in the title.

- If one of the search fields is left empty it will not be taken into account during the search.

- If $a^{\prime *}$ ' is entered in one of the fields it means as long as there is a value for 
this field (Title, Name, Description ... etc) a match will be found. Therefore to list all models in the Repository a '*' in the name field can be used; this is because all models must have a Name.

- The user is given a choice between using an AND operation or an OR operation between the search fields. For example, assume a user enters 'engine' in the title field and 'aircraft' in the Domain field. If the user selects the AND operator, then only models who have 'engine' in their title and who belong to the 'aircraft' Domain will be returned. Otherwise, if the OR operator is selected then all models with 'engine' in their title plus all models in the 'aircraft' domain will be returned. 


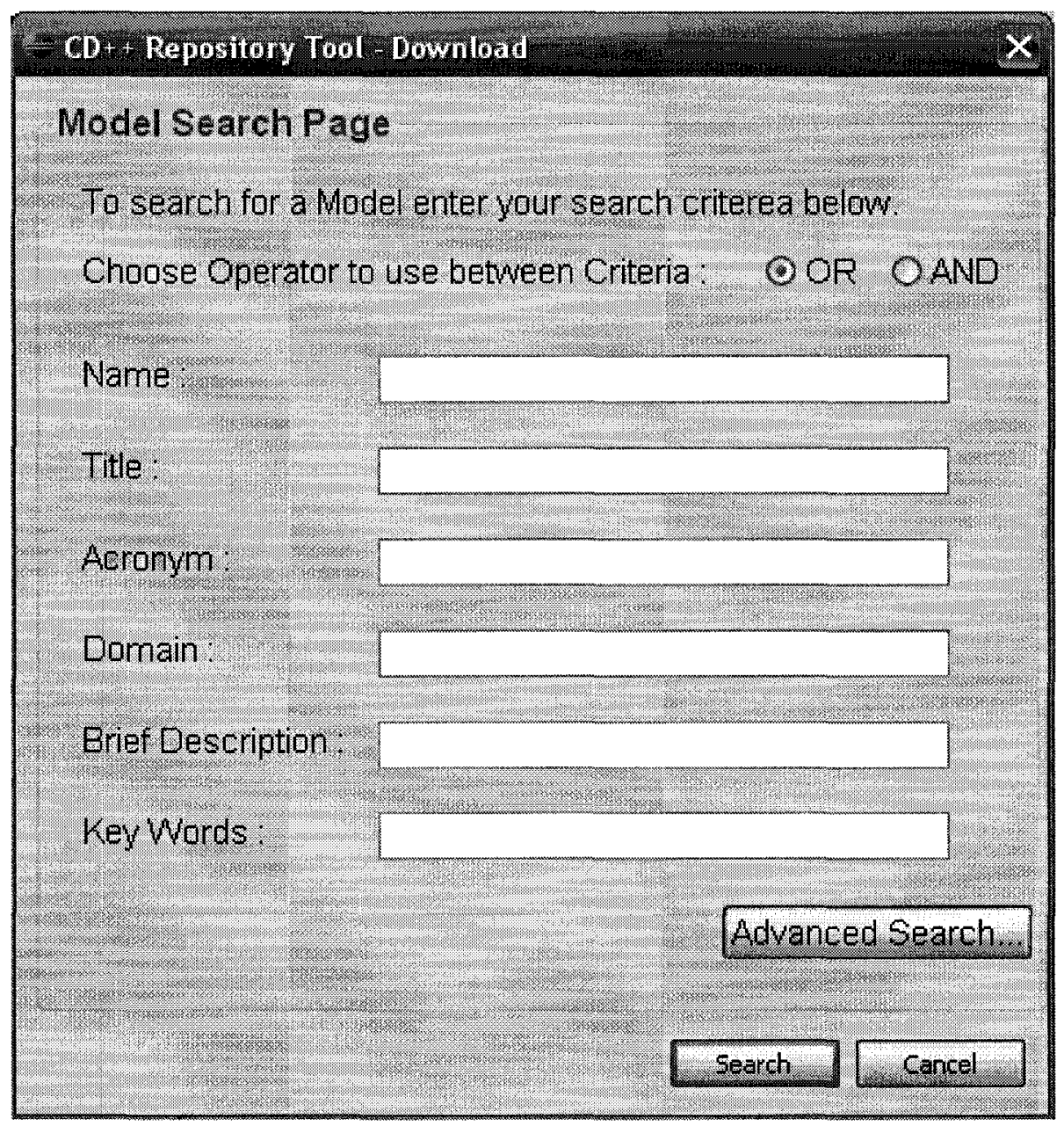

Figure 19: Model Search Window Screenshot

For more advanced search features, the user can press the "Advanced Search" button. This is intended to present a window in which more advanced search features are presented to the user. Although in the current implementation this feature is not yet implemented, an example of an important advanced search feature that can be added to this window is the ability to search for models using the Experimental Frame Data fields. 
After performing the search, the search results are presented to the user in the Search Results Window. Figure 20 shows a screenshot of the Search Results Window. As shown in the screenshot, the window is divided into three main areas: the top (referred to in the rest of this section as the Selection Pane), the bottom left (referred to in the rest of this section as the Search Results Pane), and the bottom right (referred to in the rest of this section as the Details Pane). The rest of the section describes each of these areas in more detail.

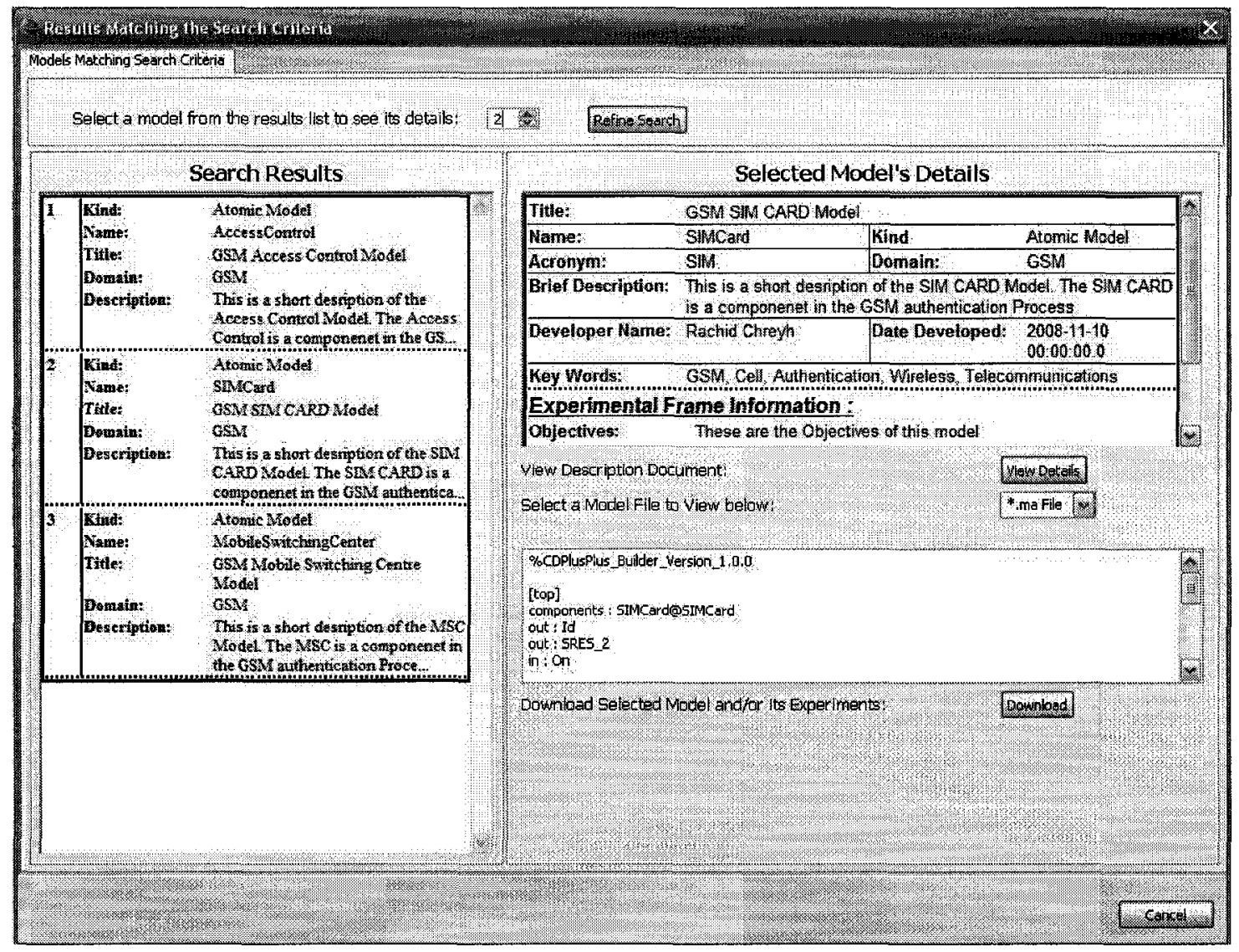

Figure 20: Search Results Window Screenshot 
The Selection Pane is where the user makes their selection of the model that interests them. It contains a drop down list and a refine search button. The drop down list is used to select one of the search results presented in the Search Results Pane, the selected item's details will be displayed in the Details Pane. The refine search is used in cases where there are too many search results returned by the original search criteria. In this case, instead of going back and re-doing the search the user can select to refine the search. When refine search is selected a window almost identical to the original search window is presented to the user. This window has all of the features of the search window except that the search is performed within the existing search results instead of being performed on the entire database. After a refine search operation is completed the search results window is refreshed with the new set of search results. The user can then proceed as normal.

The Search Results Pane contains a list of entries where each entry represents a model that matched the search criteria. As shown in Figure 20, each of these entries contains some general information about the model that it represents. This information is intended to quickly enable the user to identify the model of interest to them from among the other search results. This information consists of: 
- The kind of model (Atomic, Coupled, Cell-DEVS).

- The Name of the model.

- The Title of the model.

- The Domain of the model.

- The first few words of the Brief Description paragraph.

Finally, the Details Pane displays all of the information concerning the search result item selected by the user. At the top of the pane is an area in which all of the Model Data and Experimental Frame Data for the selected model is displayed, including a listing all of the experiments for the selected model and the experimental results for each experiment. In Figure 20 above, we can see some of the information for the model named SimCard (the user would scroll down to see the rest of this information). The View Details button enables the user to view the detailed description document for the selected model. A click of this button opens this document in an MS Word window (this requires having MS Word installed on the client PC). In fact, the user can open the description document of more than one of the search results at the same time. Finally, and perhaps most useful of all, a drop down list and a text box enable the user to view the text contained in any of the selected model's files. The user can select to display the text contained in the (.ma) (.cpp) or (.h) files by selecting the appropriate file type from the drop down list. In Figure 20 , we can see the top of the (.ma) file for the selected model. 
After looking through the search results, the user would eventually find a model that they are interested in downloading. At this point, they can proceed to the download window by pressing the download button. The download window, shown in Figure 21, allows the user to download any or all of the following items:

- The selected Model's files ((.ma) (.cpp) (.h)) and the description document.

- The child models of a Coupled model can optionally be also downloaded.

- Any or all of the Experiments linked to the selected model. By downloading an experiment, the files related to the experiment are downloaded. These include the experiment description document and for Event Based Experiments the (.ev), (.bat), (.drw), (.pal), and (.val) files, while for Model based Experiments the model files of the 'generator' and 'transducer' models. 


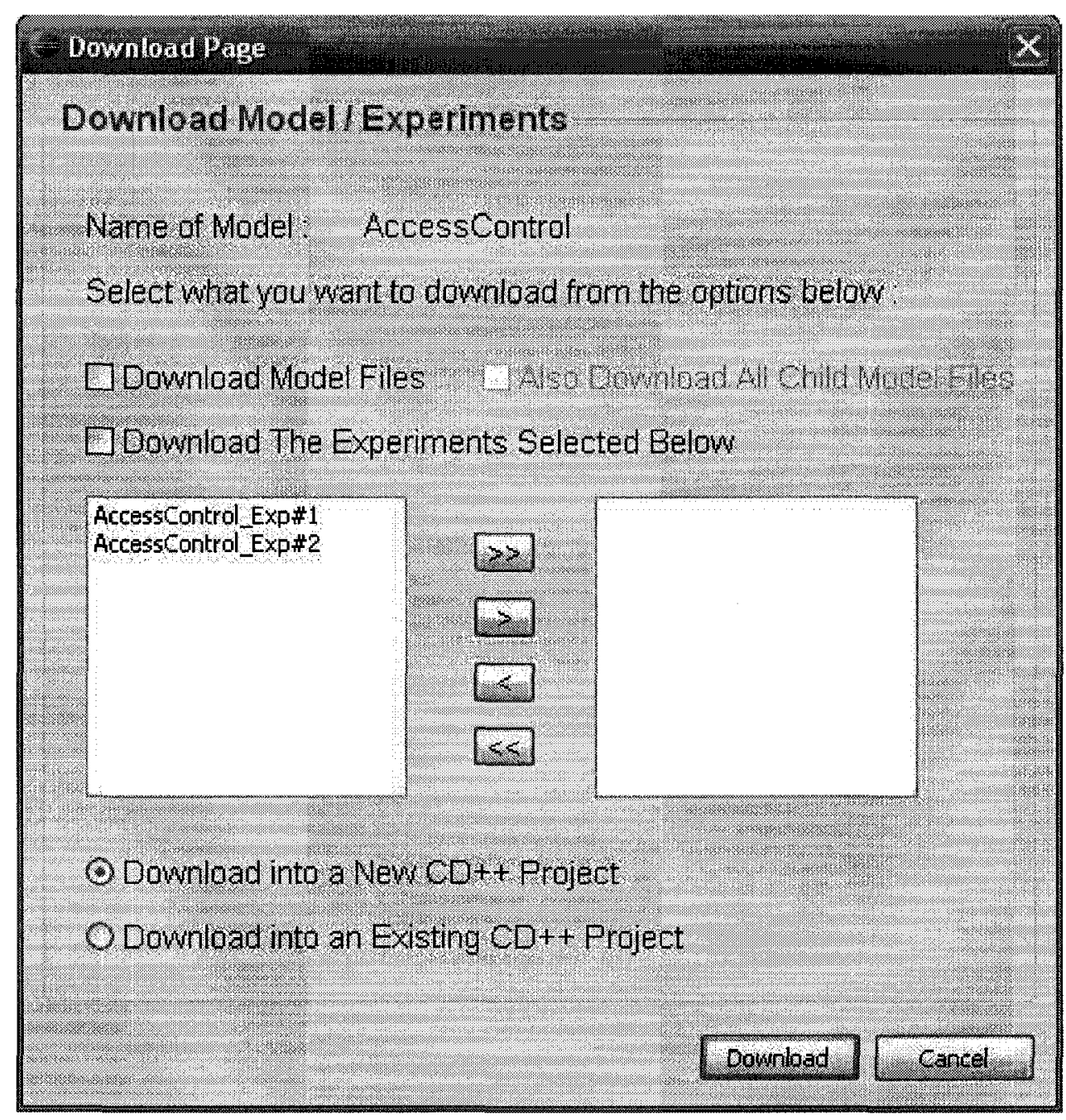

Figure 21: Download Window Screenshot

Once the download operation is started, if the user selected to download the model into a new project they will first be presented with the $\mathrm{CD}++$ new project dialogue window. After creating the new project the user is presented with the unzip wizard for each item that they chose to download. The zip wizard gives the user an opportunity to select the exact files that they want to download to their project, and gives them a chance to select a 
different project to download into. The zip wizard is used because, as was mentioned in previous chapters, all of the files related to an experiment or model are actually stored as (.zip) files on the FTP server portion of the repository's database. Once the download is complete, the use is ready to use $\mathrm{CD}++$ Builder to run or build upon the downloaded models and/or experiments.

\subsubsection{Searching for and Downloading Experiments}

The user can select to download an Experiment from the main menu of the CD++ Repository. The search dialogue window presented to the user allows the user to search for an Experiment in two ways, either using the Experiment Data to directly search for experiments in the database, or using the Model data to search for models and then create search results composed of the Experiments that are linked to the models found in the search. A screenshot of this window is shown in Figure 22 below. Note that the same search features described for the model search window apply to this search window as well. Also similar to the model search window, the user can select the Advanced Search button for a more advanced search. 


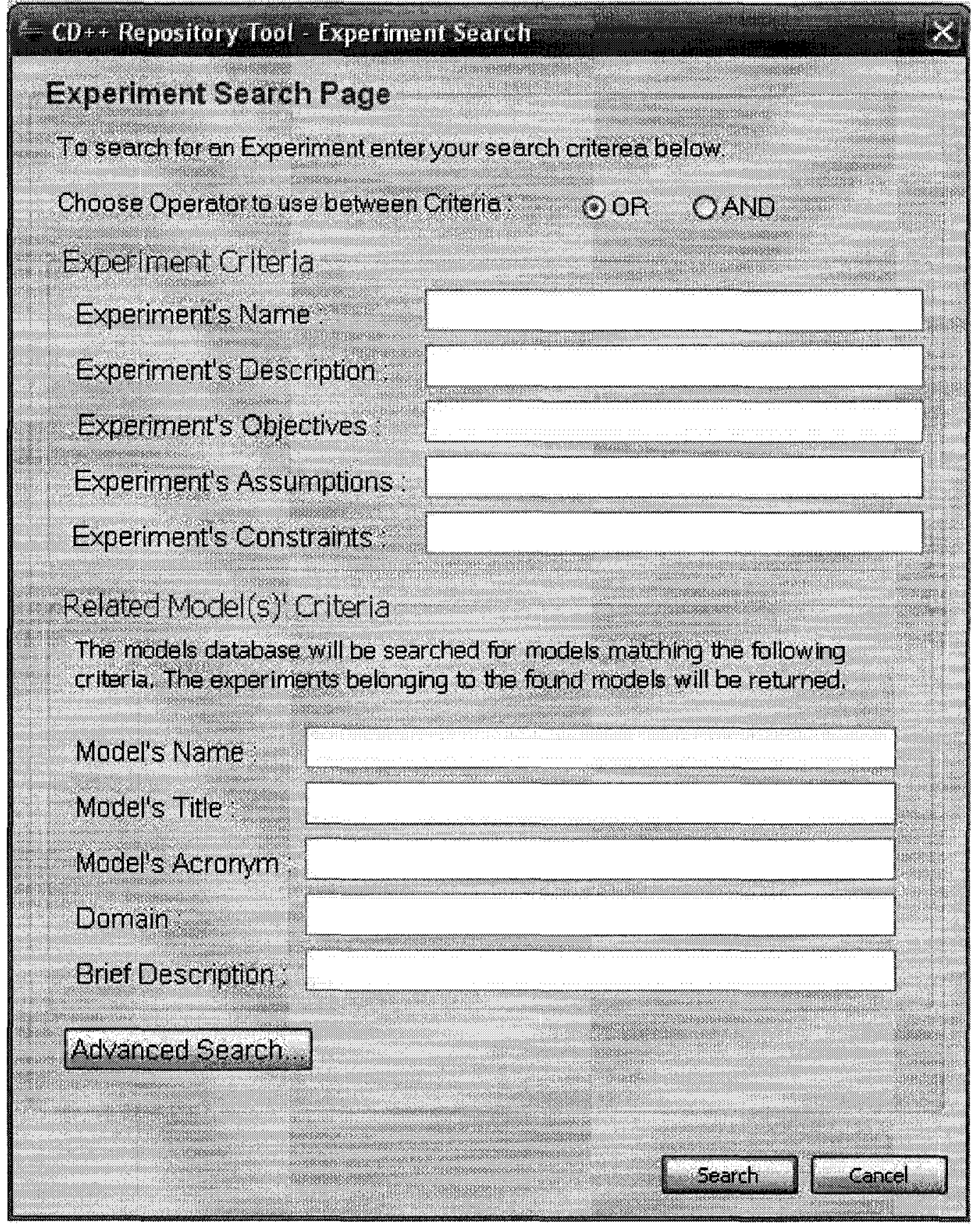

Figure 22: Experiment Search Window Screenshot

After performing the search, the search results are presented to the user in the Search 
Results Window. This window is identical to the one described in the previous section (and shown in Figure 20) for model search results except that it except that it contains experiments and experiment related information. Therefore, the Search Results Pane now contains entries representing experiments that matched the search criteria. Also, the general information presented in this pane is now composed of the following information:

- The Name of the experiment.

- The Title of the experiment.

- The kind of experiment it is (event-based or model-based).

- The first few words of the Brief Description paragraph.

Similarly, the Details Pane now contains the selected experiment's information. The area at the top of the pane now contains the Experiment Data along with the information for all of the EFs that reference the selected experiment. The rest of the Details Pane is the same as that for the model search results, except of course that the drop down list and text box now enable the viewing of the experiment files instead of the model files. When the user has found the experiment that they want to download and they press the download button, the download window, shown in Figure 23, is displayed. The window gives the user the option to download the selected experiment either into a new $\mathrm{CD}++$ project or into an existing $\mathrm{CD}++$ project. For event-based experiments, the downloaded files include the experiment description document, (.ev), (.bat), (.drw), (.pal), (.val) and any 
other files linked to the experiment, while for model-based experiments the downloaded files include the experiment description document and the model files of the 'generator' and 'transducer' models. The rest of the download operation proceeds as described in the previous section for the model download window. Once the download is complete, the user is ready to use $\mathrm{CD}++$ Builder to run or build upon the downloaded experiments.

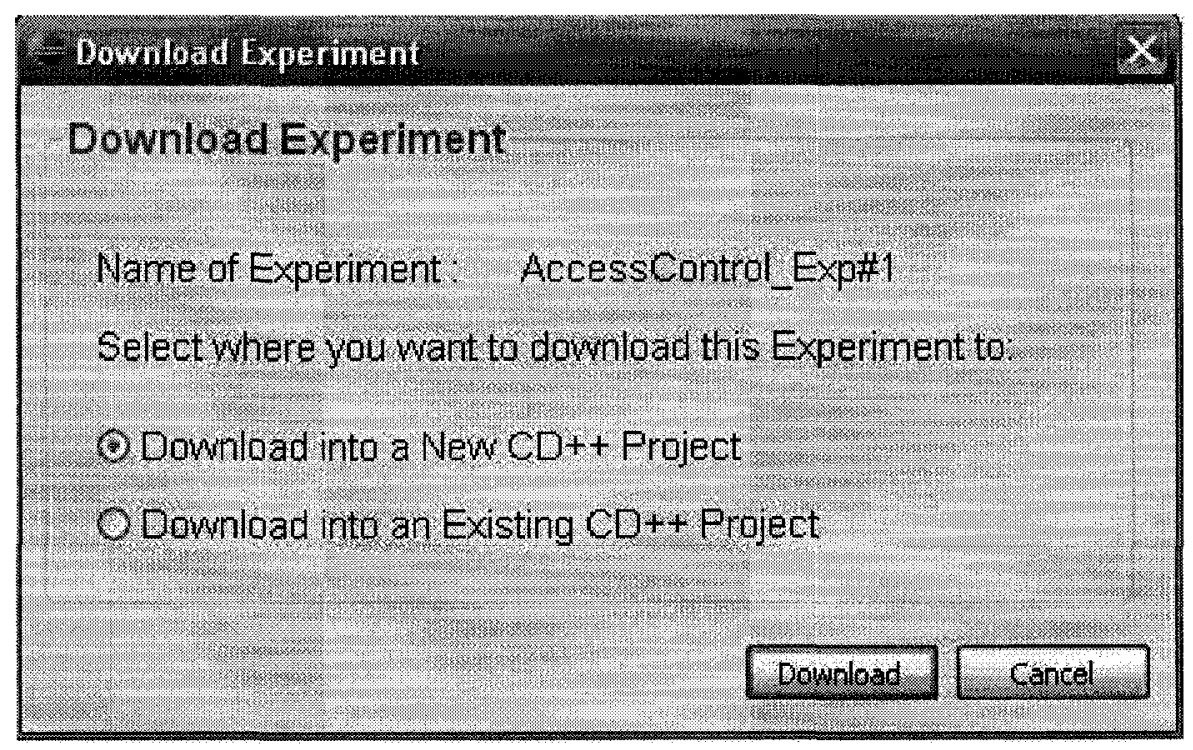

Figure 23: Experiment Download Window Screenshot

\subsection{Editing Models and Experiments}

A user can choose to modify a model or experiment that already exists in the database. The user is allowed to modify certain parts of a stored model or experiment, but there are some items that cannot be modified by the user because modifying them would amount to corrupting the database for other users. More specifically, the descriptive information 
related to a model or experiment can be modified with no adverse affects; and similarly the set of experiments related to a model can be modified (either by adding an experiment to a model's experimental frame or by removing an experiment from a model's experimental frame) also with no adverse affects. However modifying the actual $\mathrm{CD}++$ files that define the model or experiment would have a negative affect on the correctness of the database. For example suppose a model of a traffic light exists in the database and a user uses this model as a component in a model of an intersection and then the user uploads the intersection model to the database. At this point suppose that the traffic light's model definition files were modified (for example by changing it to be activated by sensors rather than on a timed basis). Now if anyone tries to download the model of the intersection they will get as part of it the new traffic light model, but the model of the intersection was designed and tested for the old model and thus more than likely the model of the intersection will now be broken.

One might ask how a user could then improve the design of an existing model. The answer for the current implementation of the $\mathrm{CD}++$ Repository is to download the model that needs improvement, modify it, and then upload the modified model as a new model with a new name. Future implementations of the $\mathrm{CD}++$ Repository could allow modifying a model or experiment by attaching a version to each stored model and experiment. By doing so the $\mathrm{CD}++$ Repository would maintain the old version of a model and add a new modified version. This way any coupled models that include the modified 
model in their hierarchy would maintain their links to the old version of the model and thus the correctness of the database would be maintained. Some prototyping work has been done with this versioning scheme and it was found that it is not too difficult to implement.

As far as the editing feature of the current implementation of CD++ Repository is concerned the user is first presented with the same search window described earlier to search for the model or experiment they intend to modify. The user is then presented with the search results window from which they can select the model or experiment that they want to modify. The user is then allowed to change any of the Model / Experiment / Experimental Frame Data. In addition the user is allowed to change the set of experiments that relate to a model and the experimental results for those experiments. After the required changes are made the user finalizes the transaction so that the modifications can be saved in the database. If the user takes too long to make a modification before finalizing their transaction and in that time the model or experiment that they are modifying was modified by another user then they will be prompted with an error message explaining what happened and are asked to redo their transaction. 


\section{Chapter 7: Testing the CD++ Repository}

The CD++ Repository tool was used to save a number of different kinds of CD++ DEVS models, and later search for and retrieve these models. The models used were randomly selected from a list of models maintained on [33]. The models shown in Table 2 were used to test the features of the CD++ Repository application. Note that although the table shows six entries, each entry represents a zip file containing a number of CD ++ DEVS Atomic, Coupled, and Cell-DEVS models.

\begin{tabular}{|l|l|l|l|}
\hline No. & Model Zip File Name & \multicolumn{1}{|c|}{ Types } & \multicolumn{1}{|c|}{ Model Structure } \\
\hline 1. & 2dHeat_Diffusion.zip & $\begin{array}{l}\text { Coupled } \\
\text { Cell-DEVS }\end{array}$ & $\begin{array}{l}\text { Coupled model containing } \\
\text { a Cell-DEVS model } \\
\text { connected to atomic } \\
\text { models as generators. }\end{array}$ \\
\hline 2. & 2dHeatConduction.zip & Cell-DEVS & $\begin{array}{l}\text { A Cell-DEVS model of 70 } \\
\text { nodes }\end{array}$ \\
\hline 3. & 3d_HeatDiffusion.zip & $\begin{array}{l}\text { Coupled } \\
\text { Cell-DEVS }\end{array}$ & $\begin{array}{l}\text { A 3 dimensional Cell- } \\
\text { DEVS model connected to } \\
\text { atomic models as } \\
\text { generators. }\end{array}$ \\
\hline 4. & Aircondition.zip & $\begin{array}{l}\text { Coupled } \\
\text { Atomic }\end{array}$ & $\begin{array}{l}\text { Coupled model containing } \\
\text { 4 atomic models (gentemp, } \\
\text { proptemp, ucontrol, and } \\
\text { ufc) and 2 coupled models } \\
\text { ( aireac and aireacon) }\end{array}$ \\
\hline 5. & Alternatebitprot.zip & $\begin{array}{l}\text { Coupled } \\
\text { Atomic }\end{array}$ & $\begin{array}{l}\text { Coupled model containing } \\
\text { a couple of child models } \\
\text { (sender and receiver) and a } \\
\text { child coupled model } \\
\text { (Network) which is made } \\
\text { of a coupled of atomic }\end{array}$ \\
\hline
\end{tabular}




\begin{tabular}{|l|l|l|l|}
\hline 6. & Atm.zip & Coupled & models (subnet) \\
\hline & Atomic & $\begin{array}{l}\text { Coupled model containing } \\
\text { 2 Atomic models } \\
\text { (cashDispenser and } \\
\text { CardReader) and a coupled } \\
\text { model (authorization) } \\
\text { which is composed of } 3 \\
\text { atomic models } \\
\text { (BalanceVerifier, } \\
\text { PINverifier, and } \\
\text { UserInterface) }\end{array}$ \\
\hline
\end{tabular}

Table 2: Table of Model Zip Files Used During Testing

For this test, the $\mathrm{CD}++$ Repository Client application was installed on a PC located outside campus and connected to the internet using a high-speed DSL modem. The SQL and FTP servers for the CD++ Repository were installed on a machine in the VSIM Building's graduate lab at Carleton University. The selected model's zip files were first downloaded from [33] to the PC where they were unzipped. Then the following aspects and features of the $\mathrm{CD}++$ Repository were tested using these models. Note that Table 3 contains tests of the features related to the models, while Table 4 contains the tests of the features related to the Experiments.

\begin{tabular}{|l|l|l|}
\hline \multicolumn{1}{|c|}{ Feature to Test } & Model Zip File Used & Result \\
\hline Uploading of a single atomic DEVS model. & $\begin{array}{l}\text { Aircondition.zip } \\
\text { Alternatebitprot.zip }\end{array}$ & Passed \\
\hline Uploading of a Cell-DEVS model. & 2dHeatConduction.zip & Passed \\
\hline
\end{tabular}




\begin{tabular}{|l|l|l|}
\hline & $\begin{array}{l}\text { 2dHeat_Diffusion.zip } \\
\text { 3d_HeatDiffusion.zip }\end{array}$ & \\
\hline $\begin{array}{l}\text { Uploading of a coupled DEVS model and } \\
\text { its children. }\end{array}$ & $\begin{array}{l}\text { 2dHeat_Diffusion.zip } \\
\text { Aircondition.zip } \\
\text { Alternatebitprot.zip } \\
\text { Atm.zip }\end{array}$ & Passed \\
\hline $\begin{array}{l}\text { Uploading of a DEVS model and its } \\
\text { experiment(s) in one operation. Also } \\
\text { uploading experimental results for each } \\
\text { experiment. }\end{array}$ & $\begin{array}{l}\text { 3d_HeatDiffusion.zip } \\
\text { Aircondition.zip } \\
\text { Alternatebitprot.zip } \\
\text { Atm.zip }\end{array}$ & Passed \\
\hline $\begin{array}{l}\text { Attempting to upload an atomic DEVS } \\
\text { model that already exists in the database. }\end{array}$ & $\begin{array}{l}\text { Aircondition.zip } \\
\text { Alternatebitprot.zip }\end{array}$ & Passed \\
\hline $\begin{array}{l}\text { Attempting to upload a coupled DEVS } \\
\text { model that already exists in the database. }\end{array}$ & $\begin{array}{l}\text { Aircondition.zip } \\
\text { Alternatebitprot.zip }\end{array}$ & Passed \\
\hline $\begin{array}{l}\text { Attempting to upload coupled DEVS } \\
\text { models containing child coupled models } \\
\text { that conflict with the repository stored } \\
\text { models. }\end{array}$ & Alternatebitprot.zip & Passed \\
\hline $\begin{array}{l}\text { Attempting to upload coupled DEVS } \\
\text { models containing child models that exist } \\
\text { in the database. }\end{array}$ & $\begin{array}{l}\text { 3d_HeatDiffusion.zip } \\
\text { Aircondition.zip }\end{array}$ & Passed \\
\hline $\begin{array}{l}\text { Atm.zip } \\
\text { by different criteria, and using OR and } \\
\text { AND operator between criteria. }\end{array}$ & $\begin{array}{l}\text { Aircondition.zip } \\
\text { 3d_HeatDiffusion.zip } \\
\text { 2dHeat_Diffusion.zip } \\
\text { Atm.zip }\end{array}$ & Passed \\
\hline $\begin{array}{l}\text { Using the refine search feature on the } \\
\text { model search results page. }\end{array}$ & $\begin{array}{l}\text { 3d_HeatDiffusion.zip } \\
\text { 2dHeatConduction.zip } \\
\text { 2dHeat_Diffusion.zip }\end{array}$ & Passed \\
\hline Verify that on the model search results & 3d_HeatDiffusion.zip & Passed \\
\hline
\end{tabular}




\begin{tabular}{|l|l|l|}
\hline $\begin{array}{l}\text { page all information of the selected model } \\
\text { is displayed correctly. }\end{array}$ & $\begin{array}{l}\text { 2dHeatConduction.zip } \\
\text { Alternatebitprot.zip }\end{array}$ & \\
\hline $\begin{array}{l}\text { Using the view details button on the model } \\
\text { search results page to display the detailed } \\
\text { description of the model (MSWord } \\
\text { document) in a separate window. }\end{array}$ & $\begin{array}{l}\text { 3d_HeatDiffusion.zip } \\
\text { Alternatebitprot.zip }\end{array}$ & Passed \\
\hline $\begin{array}{l}\text { Using the drop down list and text box on } \\
\text { the model search results page to display the } \\
\text { contents of the (.ma), (.cpp) and (.h) files }\end{array}$ & $\begin{array}{l}\text { Ad_HeatDiffusion.zip } \\
\text { Atm.zip }\end{array}$ & Passed \\
\hline $\begin{array}{l}\text { Downloading an atomic model from the } \\
\text { database. Also downloading associated } \\
\text { experiments along with the model }\end{array}$ & $\begin{array}{l}\text { Aircondition.zip } \\
\text { Alternatebitprot.zip }\end{array}$ & Passed \\
\hline $\begin{array}{l}\text { Downloading a coupled model from the } \\
\text { database. Also downloading associated } \\
\text { experiments along with the model }\end{array}$ & $\begin{array}{l}\text { Aircondition.zip } \\
\text { Alternatebitprot.zip } \\
\text { Atm.zip }\end{array}$ & Passed \\
\hline $\begin{array}{l}\text { Using the edit feature to change some } \\
\text { information of the selected model. } \\
\text { Particularly adding experiments to a } \\
\text { selected model and adding experimental } \\
\text { results to a model and experiment pair. }\end{array}$ & $\begin{array}{l}\text { Alternatebitprot.zip } \\
\text { 2dHeatConduction.zip }\end{array}$ & Passed \\
\hline
\end{tabular}

Table 3: Tests and Results for Model-Based Operations

\begin{tabular}{|l|l|l|}
\hline \multicolumn{1}{|c|}{ Feature to Test } & \multicolumn{1}{c|}{ Model Used } & Result \\
\hline $\begin{array}{l}\text { Uploading of experiments independently, } \\
\text { including the ability to upload "event- } \\
\text { based" and "model-based" experiments. }\end{array}$ & $\begin{array}{l}\text { 2dHeatConduction.zip } \\
\text { Atm.zip }\end{array}$ & Passed \\
\hline $\begin{array}{l}\text { Using the search capability to find } \\
\text { experiments by different criteria, and using } \\
\text { OR and AND operator between criteria. }\end{array}$ & $\begin{array}{l}\text { 2dHeatConduction.zip } \\
\text { Atm.zip }\end{array}$ & Passed \\
\hline $\begin{array}{l}\text { Using the refine search feature on the } \\
\text { experiment search results page. }\end{array}$ & 2dHeatConduction.zip & Passed \\
\hline
\end{tabular}




\begin{tabular}{|l|l|l|}
\hline & Atm.zip & \\
\hline $\begin{array}{l}\text { Verify that on the experiment search results } \\
\text { page all information of the selected model is } \\
\text { displayed correctly. }\end{array}$ & $\begin{array}{l}\text { 2dHeatConduction.zip } \\
\text { Atm.zip }\end{array}$ & Passed \\
\hline $\begin{array}{l}\text { Using the view details button on the } \\
\text { experiment search results page to display } \\
\text { the detailed description of the experiment } \\
\text { (MSWord document) in a separate window. }\end{array}$ & $\begin{array}{l}\text { 2dHeatConduction.zip } \\
\text { Atm.zip }\end{array}$ & Passed \\
\hline $\begin{array}{l}\text { Using the drop down list and text box on the } \\
\text { experiment search results page to display } \\
\text { the contents of the experiment files (such as } \\
\text {.ev, .pal ... etc). }\end{array}$ & $\begin{array}{l}\text { Aircondition.zip } \\
\text { Atm.zip }\end{array}$ & Passed \\
\hline $\begin{array}{l}\text { Downloading an experiment, whether it is } \\
\text { event-based or model-based, from the } \\
\text { database }\end{array}$ & $\begin{array}{l}\text { 2dHeatConduction.zip } \\
\text { Atm.zip }\end{array}$ & Passed \\
\hline $\begin{array}{l}\text { Using the edit feature to change some } \\
\text { information of an experiment }\end{array}$ & $\begin{array}{l}\text { Aircondition.zip } \\
\text { Atm.zip }\end{array}$ & Passed \\
\hline
\end{tabular}

Table 4: Tests and Results for Experiment-Based Operations.

The last area of testing for the $\mathrm{CD}++$ Repository is to make sure that concurrent modification to the same models at the same time does not cause a corruption of the database. There are two scenarios where such concurrent modifications may take place; the first is when two users, each with their own CD++ Repository Client, try to edit the same model at the same time. In this case, the transaction of the first user to finish editing will be committed to the database, and when the second user tries to commit their change they will be presented with an error message and asked to re-do their editing. The second scenario is when two users want to create a mode with the same name at the same time. 
In this case, the user who finishes selecting their (.ma) file first will essentially win the race and the second user will be told that a model with the same name already exists.

To test these scenarios the CD++ Repository Client application was installed on another PC also located outside campus and also connected to the internet using a high speed DSL modem. To test the first scenario the steps in Figure 24 were followed, and to test the second scenario the steps in Figure 25 were followed. Testing showed that in both scenarios the software preformed as expected.

- Concurrent editing of repository elements:

○ On PC \#1 start editing a model with name "foo".

○ On PC\#2 start editing the same model "foo".

○ Complete editing of "foo" from PC\#1.

- Attempt to complete editing from PC\#2.

- An error message is given asking user to redo editing.

Figure 24: Concurrent editing of Models Test 
- Concurrent upload of repository elements with same name:

O On PC\#1 start uploading a model with name "foo".

- On PC\#2 start uploading a model with name "foo".

O On PC\#1 finish selecting the (.ma) file for the model.

O On PC\#2 try to upload the same model "foo".

- An error message is given saying "foo" already exists.

Figure 25: Concurrent Upload of Models with Same Name Test 


\section{Chapter 8: Conclusion and Future Work}

DEVS modelling and simulation environments would benefit greatly if they were associated with a repository of DEVS models and experiments. Users of these simulation environments could then share and reuse models and experiments. In addition, the availability of this repository from any geographical locations allows teams of modellers that are far away from each other to collaborate in their work.

In this thesis, we presented an architecture for an Internet-based repository of DEVS models and experiments. It was shown that earlier work related to the creation of model repositories was not suited for adequate model reuse. The architecture presented here had several contributions. The first is the idea of storing, along with the models, the EF for these models. The EF holds the context of use information for the model and contains the experiments applicable to the model. This allows users of the repository to reuse models and experiments. In addition, the repository stores the experimental results thus allowing users to reproduce results and thus verify the fidelity of the models. The second is in specifying the exact storage entities that need to be created, the relationships between these entities and the information that each entity should include. Thirdly, the proposed architecture enables the creation of "open source" repositories where users from all over the world could connect to, upload, and download items to/from the repository. Finally, by being general enough in its design, the architecture is a small step towards allowing collaboration among users of different DEVS tools. 
A prototype application based on the proposed architecture was implemented for the $\mathrm{CD}++$ Builder Toolkit. This application, $\mathrm{CD}++$ Repository, was presented in this thesis and tested to show how the proposed architecture could work in a real application. Using the $\mathrm{CD}++$ Repository one can easily follow the theoretical modeling process presented in [12] to build complex models.

\subsection{Future Work}

The proposed repository architecture provides a step in the direction of enabling DEVS modellers to reuse models in their endeavour to build models that are more complex and thus enabling teamwork in this area. Future work in this area however can improve on the current abilities and features of this application. Some of the areas on which work can be done include:

1. The current repository architecture uses relational database tables to store all of the information in the library. An important research area would be to replace the existing relational database with a database based on the OWL ontology language. This means major changes to the repository servers. It will require building OWL ontology for the DEVS models and their Experimental Frames. Achieving this will allow users to use semantics in their queries of the database and will provide a more powerful search capability. The area of OWL and the semantic web is a new and expanding area of research. 
2. The current architecture of the repository is intended to be used for a single DEVS simulation tool. This is because the model files stored in the repository are specific to a given tool. Research is required to somehow store a general format of the DEVS model in the repository and thus allow any DEVS simulation tool to download all of the stored models. This would allow true interoperability between the different DEVS simulation tools.

3. The architecture presented in this work supports an "Open-Source" concept. This means that any user from anywhere on the internet can access the repository and upload files to it. While having a repository that is open to anyone to contribute to is desirable, this feature also presents some security risks. For example, a malicious user may upload viruses or other undesirable or illegal items to the repository. The current architecture does not adequately address these security problems. An important avenue for future work could be to address these security problems in ways that do not hinder the ability of legitimate users in accessing and uploading items to the repository. 


\section{References}

[1] Ted Biggerstaff and Alan Perlis (Eds). 1989. Software Reusability, volume 1 \& 2. ACM Press, NY.

[2] Zeigler, B.; H. Praehofer; T.G. Kim. 2000. Theory of Modeling and Simulation, $2^{\text {nd }}$ Edition. Academic Press, San Diego, CA.

[3] Traore M.K., and A. Muzy. 2006. "Capturing the Dual Relationship Between Simulation Models and Their Context." Simulation Modeling Practice and Theory, Vol. 14, No.2, (February): 126-142

[4] Chidisiuc K. and G. Wainer. 2008. "CD++Modeler: A Graphical Toolkit to Develop DEVS Models." Poster Paper. In Proc. of SpringSim'08, April 14-17, in Ottawa, ON, Canada.

[5] Chreyh R. and G. Wainer. 2009. "CD++ Repository: An Internet Based Searchable Database of DEVS Models and Their Experimental Frames." In Proceedings of SpringSim'09, March 23-25, in San Diego, CA, USA.

[6] Macleod M.; R. Chreyh; G. Wainer. 2006. "Improved cell-DEVS Models for Fire Spreading Analysis." In Proceedings of the 7th International Conference on Cellular Automata for Research and Industry, ACRI 2006, September 20-23, in Perpignan, France. Vol. 4173: pp. 472-481.

[7] Bernardi F., J.-B. Fillipi, and J.-F. Santucci. 2003. "A Generic Framework For Environmental Modeling and Simulation." In Proceedings of the 2003 IEEE 
International Conference on Systems, Man and Cybernetics, October 5-8, in Washington, DC. 1810-1815 Vol. 2.

[8] Wainer G.; N. Giambiasi. 2001. “Timed Cell-DEVS: Modeling and Simulation of Cell Spaces." Invited paper for the book Discrete Event Modeling \& Simulation: Enabling Future Technologies. Springer-Verlag.

[9] Zeigler, B. 1984. Multifaceted Modeling and Discrete Event Simulation. Academic Press, London.

[10] Barros F. J.; A. Lehmann; P. Liggesmeyer; A. Ver-braeck; B. P. Zeigler. 2006. “04041 Abstracts Collection -- Component-Based Modeling and Simulation.” In Proceedings of Dagstuhl Seminar 04041 Component-Based Modeling and Simulation, Jan. 1, in Dagstuhl, Germany.

[11] Bernardi, F.; J.F. Santucci. 2002. "Developing a Web-Based Models Library for a DEVS Modeling and Simulation Environment." In Proceedings of AIS 2002, April 7 -10 , in Lisbon, Portugal.

[12] Bernardi, F.; J.F. Santucci. 2002. "Model Design Using Hierarchical Web-Based Libraries." In Proceedings of the $39^{\text {th }}$ Conference on Design Automation, June 9-14, New Orleans, USA. Vol. 1: pp. 14-17.

[13] Bernardi, F.; E. de Gentili; and J. Santucci. 2001. "Reusable Models Integration in a DEVS-Based Modelling and Simulation Environment." In Proceedings of ESS2001, Oct. 18-20, Marseille, France. Vol. 1: pp. 644. 
[14] Mocko, G.; Malak Jr., R. J.; Paredis, C. J. J.; and Peak, R. 2004. "A Knowledge Repository for Behavioral Models in Engineering Design." In Proceedings of the 24th ASME Computers and Information in Engineering Conference, Sept. 28-Oct. 2, Salt Lake City, UT, ASME DETC2004-57746; Vol. 4: pp. 943-952.

[15] Breunese, A. P. J.; Top, J. L.; Broenink, J. F.; and Akkermans, J. M. 1998. "Libraries of Reusable Models: Theory and Application." Simulation. Vol. 71, (July): pp. 7 - 22.

[16] Garrido, J. M.; and Amit, J. 2005. "A Repository for Multi-Disciplinary Computational Models and Tools." In Proceedings of the 43rd annual Southeast Regional Conference, March 18-20, Kennesaw, Georgia. Vol. 1: pp. 315-316.

[17] Balci, O. 1998. "A Library of reusable Model Components for Visual Simulation of the NCSTRL System." In Proceedings of the 1998 Winter Simulation Conference, Dec. 13-16, Washington DC. pp. 1451-1460.

[18] Son, Y. J.; Jones, A.T.; Wysk, R.A. 2000. "Automatic Generation of Simulation Models from Neutral Libraries: An Example." In Proceedings of the 2000 Winter Simulation Conference, Dec.10-13, Wyndham Palace Resort \& Spa, Orlando, FL. pp. $1558-1567$.

[19] Praehofer, H.; Sametinger, J.; and Stritzinger, A. 2000. "Building Reusable Simulation Components." In Proceedings of WEBSIM2000, Web-Based Modelling \& Simulation, Jan 23-27, San Diego, CA, USA. Vol. 1: pp. 1-7. 
[20] NS-2 website. Available at: http://nsnam.isi.edu/nsnam/index.php/User_Information. [Accessed March, 2009]

[21] OMNeT++ website. Available at: http http://www.omnetpp.org. [Accessed March, 2009]

[22] OPNET Technologies Inc. website. Available at: http://www.opnet.com. [Accessed March, 2009]

[23] Wainer, G. DEVS Tools website. Available at: http://www.sce.carleton.ca/faculty/ wainer/standard/tools.htm. [Accessed March, 2009]

[24] Nutaro, J. ADEVS website. Available at: http://www.ornl.gov/ 1qn/adevs. [Accessed January, 2009]

[25] Zeigler, B.; Y. Moon; D. Kim; J. G. Kim. 1996. "DEVS-C++: A high performance modeling and simulation environment." In The $29^{\text {th }}$ Hawaii International Conference on System Sciences, Jan. 3-6, Maui, Hawaii. pp. 350-359.

[26] Zeigler, B.; H. S. Sarjoughian. 1999. "Support for hierarchical modular componentbased model construction in DEVS/HLA." Simulation Interoperability Workshop, March 14-19, Orlando, FL.

[27] Sarjoughian, H. S. and B. Zeigler.1998. "DEVSJAVA: Basis for a DEVS-based collaborative M\&S environment." In Proceedings of The International Conference on Web-Based Modeling and Simulation, Jan. 11-14, San Diego, CA. USA. Vol. 5: pp. 29-36. 
[28] Filippi, J. B.; F. Bernardi; M. Delhom. 2002. "The JDEVS Modeling and Simulation Environment'. In Proceedings of the Integrated Assessment and Decision Support Conference (IEMSS'02), Jun. 24-27, Lugano, Switzerland. pp. 283-288.

[29] Praehofer, H.; J. Sametinger; A. Stritzinger. 1999. "Discrete Event Simulation Using the JavaBeans Component Model." In Proceedings of the International Conference on Web-Based Modeling \& Simulation, Jan. 17-20, San Francisco, CA. USA.

[30] Bauer C.; G. King. 2005. Hibernate In Action. Manning Publications Co., Greenwich, CT.

[31] Weathersby J.; D. French; T. Bondur; J. Tatchell; I. Chatalbasheva. 2006. Integrating and Extending BIRT. Addison-Wesley, New York.

[32] Wainer, G; L. Morihama; V. Pasuello. 2002. "Automatic Verification of DEVS Models." In Proceedings of 2002 Spring Simulation Interoperability Workshop, March 10-15, Orlando FL,

[33] Wainer, G. DEVS Tools website. Available at: http://www.sce.carleton.ca/faculty/ wainer/wbgraf/samplesmain _1 [Accessed March, 2009] 


\section{Appendix-A: CD++ Repository Software Details}

\section{Persistence Layer Java Packages}

In the previous section Hibernate was introduced as a layer that sits at the interface with the database and handles the mapping of the Business Objects to the database tables. As such Hibernate is a major part of the persistence layer of the CD++ Repository. The use of Hibernate does make things easier in terms of solving the object-relational mismatch problem, but one must have a good understanding of Hibernate in order to use it correctly in a Java application. One main requirement for the use of Hibernate is that the objects being persisted must be attached to a Hibernate session for Hibernate to be able to save them to the database. Objects that are attached to a session are called persistent objects, while those not attached to a session are called either detached objects if they were attached to a session that is now closed, or transient objects if they have not been attached to a session yet. Another thing to note about Hibernate is the session and transaction demarcation issues; that is when to open a session and when to close it, and when to start a transaction and when to commit it. For example, one should not have a Hibernate session or transaction open during user interaction. Other concepts one needs to understand when using Hibernate include transitive persistence strategies, concurrency and automatic versioning, and even caching options for optimizing performance. The CD++ Repository's architecture tries to hide most of the details that deal with the use of Hibernate inside the Persistence Layer. Therefore a few packages and utilities have been built to encapsulate the database services required by the Presentation layer such that 
Presentation layer code is not bogged down with Hibernate programming details. The next sub-sections introduce these Persistence layer packages and utilities.

\subsection{The HibernateUtil Class}

This is the utility used to start Hibernate. It contains a static SessionFactory attribute and a static getter function for it. An initialization method called InitializeSessionFactory() exists to instantiate and configure the Hibernate SessionFactory by loading configuration information from the Hibernate configuration file as well as using the CD++ Repository preferences page to get information required to connect to the appropriate SQL server and database. The SessionFactory is instantiated only once when the CD++ Repository is first started and it is used throughout the life of the application. Other utility methods that are made available to the Presentation Layer by this class are:

- attachToSession(): Used to attach a detached object to a session. This is useful in situations where the object being attached contains a graph of other objects whose values need to be accessed from the database.

- commitSessionTx(): Used to end the transaction and close the session started by $\operatorname{attachToSession}(0$.

- mergeObject(): This method opens a session, updates (merges) the database with the passed in detached object's information, and closes the session. 


\subsection{The RepositorySearchUtil Class}

This utility provides the following methods that allow inspection of the repository with respect to a given Model or Model name:

1. isInConflictWithRepo(): This method determines whether a passed in coupled model has the same structure (i.e. the same number of atomic and coupled child models) as the matching coupled model (by name) in the repository.

2. modelNameInRepository(): This method determines if the passed in model name already exists in the repository.

3. getAtomicModelFromRep $($ and getCoupledModelFromRep: These two methods search the repository for a model matching the passed in model name and return the model object if it is found.

\subsection{The dataAccessObjects Package}

This package contains a data access class for each business object. This means that there is an ExperimetDAO class, a ModelDAO class, an ExperimentalFrameDAO and so on. The data access classes are intended to hide the details of how the database is accessed and searched to obtain an object from the database. Each class contains a number of methods intended to facilitate searching for items in the database or adding items to the database. The following are examples of the types of methods in the DAO classes: 
- Search by attribute methods. These are intended to search the database based on a specific attribute of the business object in question. These methods take in a search string and return a set of objects that matched the search string in the database. For example the modelDAO class contains, among other methods, the following:

- findByDescription(): This method searches the database and returns all models whose description attribute contains the passed in string.

- findByTitle(): This method searches the database and returns all models whose title attribute contains the passed in string.

- Search by attribute and set-of-object-names methods. These methods are similar to the ones described above except that in addition to the search string a set of object names is passed in. These methods are used to search the database based on a specific attribute and based on the set of names that are passed in. These methods are useful when doing refine search operations.

- Add methods. These methods are used to add a given object, or map of objects to the database.

The DAO classes hide the Hibernate Query Language (HQL) being used to do the searching in the database and hide the implementation of the search and add methods. This separation of concerns promotes maintainability of the software so that if 
improvements are to be implemented to the search algorithm for example it will only affect the DAO classes. Note that these DAO Classes are not intended to be called directly by the presentation layer. The next section introduces the databaseServices package which contains wrappers to the methods in the DAOs and these are the ones that the Presentation layer accesses.

\subsection{The datbaseServices Package}

The databaseServices package also contains a class for each business object. Each class provides database services for the related business object. The majority of the methods in these classes are similar to the methods of the DAO classes. In fact each of the search and add methods of the DAO classed is encapsulated by a similar method in the corresponding databaseService class. In addition some of the databaseService classes contain methods that are used to perform more complicated functions such as more advanced searches of the database. The more important of these methods are listed below:

- The findModels 0 method in the ModelService class is used by the Presentation layer to find the models, both coupled and atomic, that match certain search criteria that are passed into it. This method takes in a list of criteria such as name, description, title, and a corresponding list of strings for each criterion. In addition it takes in an indicator to whether an OR or an AND operation should be used 
between these search criteria. This method makes use of the DAO classes and methods to perform the search in the database, and returns a list of models that match all of the search criteria using the appropriate operator.

- The refineModels 0 method in the ModelService class is used by the Presentation layer to do a "refine search" operation on a set of Model search results. This method takes in a list of criteria such as name, description, title, a corresponding list of strings for each criterion, and a set of names of the models to do the refine search on. In addition it takes in an indicator to whether an OR or an AND operation should be used between the search criteria. This method makes use of the DAO classes and methods to perform the refine search, and returns a list of models that match all of the search criteria.

- The findExperiments 0 method in the ExperimentService class is used by the Presentation layer to find the Experiments that match certain search criteria that are passed into it. This method is very similar to the findModels( ) method.

- The refineExperiments( ) method in the ExperimentService class is used by the Presentation layer to do a "refine search" operation on a set of Experiment search results. This method is very similar to the refineModels() method.

- The createExpFrameWithInputsOutputs 0 method is found in the ExperimentalFrameService class and is used by the Presentation layer when storing the Experimental Frame's input and output port information. This method 
creates a new experimental frame object and assigns to it the values for the inputs and outputs that were entered by the user. It checks the database for existing input/output elements. If an element of an input or output is found in the database, then that element is itself used in the current experimental frame object without having to create a new one, otherwise if it is not found in the database it is added to the database and then used in the current experimental frame object. This way no redundancy exists in the database for input/output elements.

\section{2- Presentation Layer Packages}

Since the $\mathrm{CD}++$ Repository is a component of the $\mathrm{CD}++$ Builder Toolkit, which in itself is an Eclipse plug-in, the interface to the $\mathrm{CD}++$ Repository has to be integrated with the rest of the $\mathrm{CD}++$ Builder Toolkit plug-in and should have a similar look and feel as the Eclipse environment. To that end the Presentation layer of the $\mathrm{CD}++$ Repository is mostly built using the Eclipse Standard Widget Toolkit (SWT) package. Also, to complete the integration, the user preferences for the $\mathrm{CD}++$ Repository application are integrated in the Eclipse preferences menu under the $\mathrm{CD}++$ Builder Preferences section. Finally, launching the $\mathrm{CD}++$ Repository application is done through a button in the CD++ Builder toolbar in Eclipse.

Once the $\mathrm{CD}++$ Repository is launched the user is presented with the main $\mathrm{CD}++$ Repository window which allows the user to perform the two main functions of the 
CD++ Repository; namely uploading items to the database, and searching for and downloading items from the database. Therefore there are two main presentation layer packages, the first contains the classes concerned with uploading and the second contains classes that are concerned with searching and downloading. The next few sections explain the structure of these packages in more detail. Note that the CD++ Repository does also allow the user to edit items in the repository, but this is essentially a combination of the previous two functions, first an item is downloaded and then edited and uploaded back to the repository.

\section{1- The Gui.Upload Package}

This package is the root container of all the user interface classes that deal with the uploading of items to the database. This package in itself does not contain any classes, but it contains a number of packages, one for each item that can be uploaded to the $\mathrm{CD}++$ Repository's database. Figure 26 below shows that under this package there are the following child packages: model, expFrame, experiment, and experimentalResult; these are explained in the following sub-sections. 


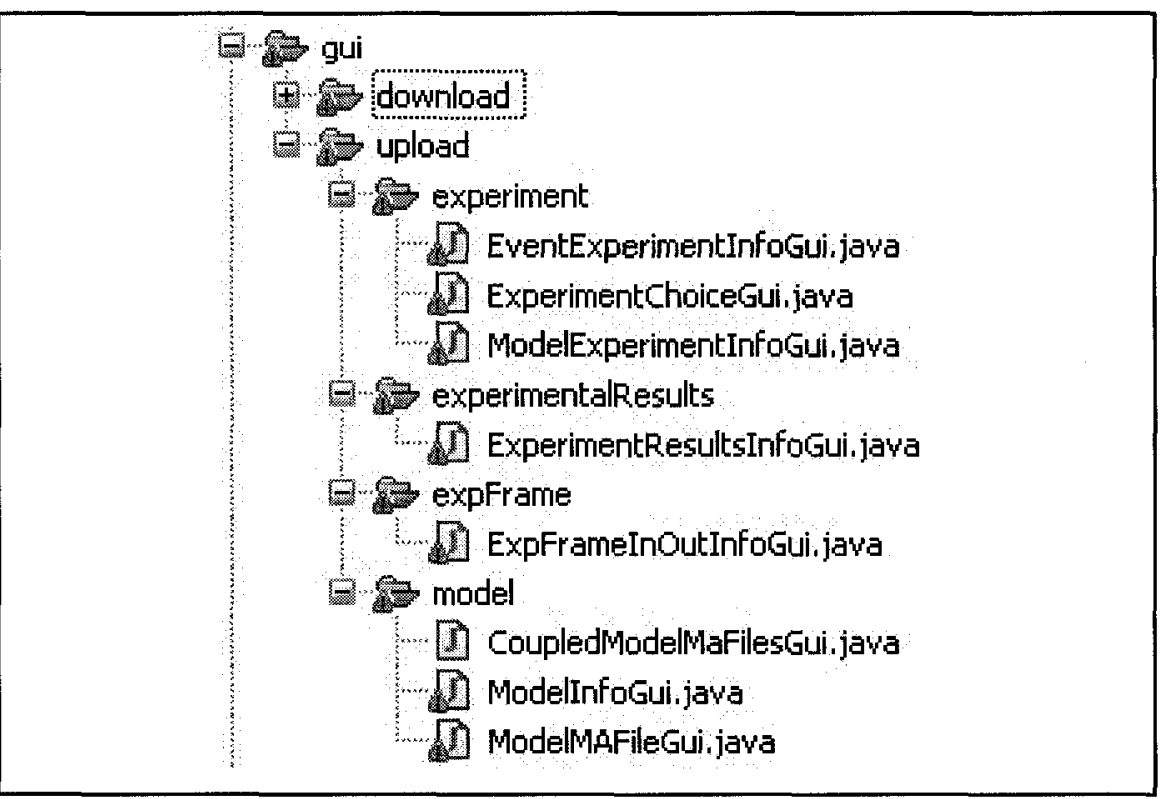

Figure 26: The gui.upload Java Package Structure

\subsection{1- The Gui.Upload.Model Package}

As indicated by its name, this package contains all of the classes that produce and manage the user interface windows responsible for uploading models (both Coupled and Atomic) to the database. The following is a brief description of these classes:

- ModelInfoGui: This class extends the eclipse.jface.dialogs.Dialog class and is responsible for drawing the GUI that collects the user entered information regarding the model being uploaded. This information is composed of all of the Model Data and Experimental Frame Data for the model, in addition to the paths for all of the files that have to be uploaded for the model such as the (.ma), (.h), and (.cpp) files. This class simply collects the information into a business object 
which can then be processed further by the code that instantiated and ran this class. The user can navigate to the Experiment download window and the Experimental Frame additional information upload window from this class as well.

- CoulpedModelMaFileGui: This class is also based on the eclipse.jface.dialogs.Dialog class and it is responsible for collecting the (.ma) file paths for the list of model names passed into it. The file paths entered by the user are stored in a map with corresponding model names, and this map can be retrieved by the code that instantiated this class.

- modelMaFileGui: This class also extends the eclipse.jface.dialogs.Dialog class, and it is the main entry and control point for all of the model upload process. The user interface window drawn by this class is simply a text box and a button to allow the user to enter the path of the (.ma) file for the model that is to be uploaded. When the user clicks the button, this class calls the appropriate utility and database service classes to parse the (.ma) file, determine the type of model being uploaded, determine all the child models (if any), and check for conflicts with the database as described in earlier chapters, and, in the case of a coupled model, create a list of the new child models that the user is to enter. If child coupled models are detected then the CoulpedModelMaFileGui class is instantiated to collect the paths of the (.ma) files for each of those child coupled models. Finally the ModelInfoGui class is instantiated to collect the model 
information. In the case where a coupled model is being downloaded, then the ModelInfoGui class is instantiated recursively for all child models that do not already exist in the database.

\subsection{2- The Gui.Upload.Experiment Package}

This package contains all of the classes that produce and manage the user interface windows responsible for uploading experiments to the database. The following is a brief description of these classes:

- ExperimentChoiceGui: This class extends the eclipse.jface.dialogs.Dialog class and is the main entry point for uploading experiments. The window displayed by this class gives the user an opportunity to choose the type of Experiment they want to upload; either an event file based experiment, or a model based experiment. Depending on the choice one of the classes described below is instantiated to collect the Experiment's information.

- EventExperimentInfoGui: This class extends the eclipse.jface.dialogs.Dialog class and is responsible for drawing the GUI that collects the user entered information for event file based experiments. This information is composed of all of the Experiment Data, in addition to the paths for all of the files related to the experiment such as the (.ev) and (.doc) files. This class simply collects the information into a business object which can then be processed further by the code that instantiated this class. After entering the Experiment's information the 
user is presented with Experimental Results upload window to enter the experimental result information if any.

- ModelExperimentInfoGui: This class extends the eclipse.jface.dialogs.Dialog class and is responsible for drawing the GUI that collects the user entered information for model based experiments. This information is composed of all of the Experiment Data in addition to all of the model information for the model that will act as a generator and the model that will act as a transducer. The model information is collected either by invoking the ModelInfoGui class to collect information of a new model, or by invoking the ModelSearchGui class to search for and select an existing model in the database. This class collects the information into a business object which can then be processed further by the code that instantiated this class. After entering the Experiment's information the user is presented with Experimental Results upload window to enter the experimental result information if any.

\subsection{3- The Gui.Upload.ExperimentalResults Package}

This package contains only one class, the ExperimentalResultsInfoGui, which is responsible for collecting the experimental results information from the user. This class collects the required information into a business object which can then be processed further by the code that instantiated this class. 


\subsection{4- The Gui.Upload.ExpFrame Package}

This package contains only one class, the ExpFrameInOutInfoGui, which is responsible for collecting the experimental frame's other information. Note that the regular Experimental Frame Data is collected by class ModelInfoGui. As mentioned in previous chapters, this other information is composed of the inputs and outputs of the experimental frame as described by the formalism in [3]. Collection of this information in the current version of the $\mathrm{CD}++$ Repository is not required for the rest of the functionality of the tool to operate. This development was done as an example of how the $\mathrm{CD}++$ Repository can capture information at this level of detail, future development of the CD++ Repository could make use of this information to more fully describe the interaction of a given model with other models. This class collects the required information into business objects which are then added to the Experimental Frame object by the code that instantiated this class.

\section{2- The Gui.Download Package}

This package is the root container of all the user interface classes that deal with collecting the search criteria from the user, displaying the search results to the user, and downloading the selected item(s) from the database to a $\mathrm{CD}++$ Builder project. This package in itself does not contain any classes, but it contains a package for models and another for experiments (the two entities that can be downloaded from the database) and 
a couple of other supporting packages. Figure 27 shows the hierarchy under this package and the following sub-sections give a brief description of the packages in this hierarchy.

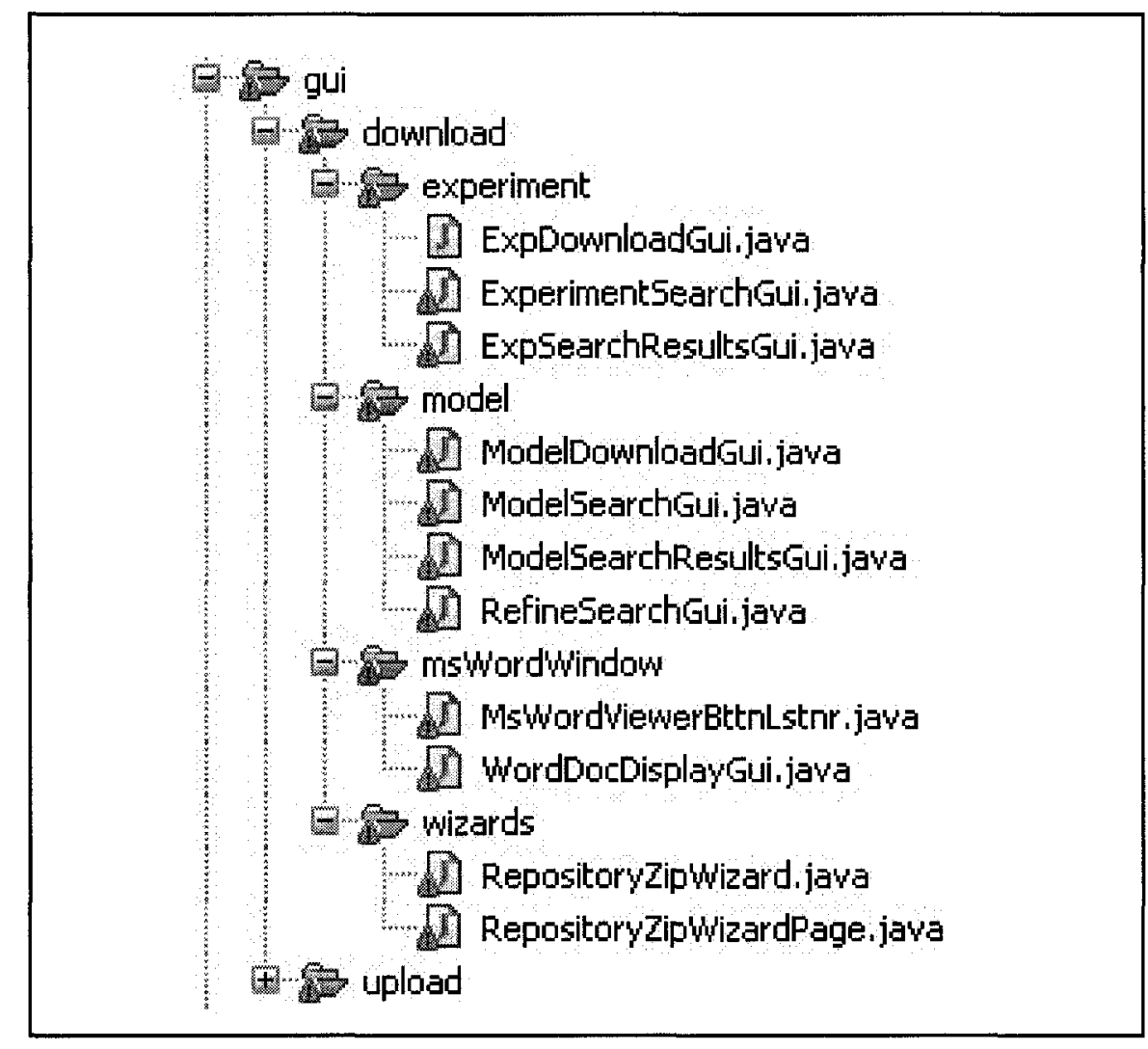

Figure 27: The gui.download java Package Structure

\subsection{1- The Gui.Download.Model Package}

This package contains all of the classes that produce and manage the user interface windows responsible for collecting search criteria, displaying search results and 
downloading models (both Coupled and Atomic). The following is a brief description of these classes:

- ModelSearchGui: This class extends the eclipse.jface.dialogs.Dialog class and is the entry point to the download functionality. It creates the user interface window responsible for collecting the search criteria and the other search options from the user. When the user enters the search criteria and presses the search button on this user interface window, the class calls the appropriate search routines from the Persistence Layer to do the actual search in the database. These search routines return a list of search results in the form of the business objects of the matching items. The role of this class ends by instantiating the ModelSerachResults class and passing it the list of business object search results.

- RefineSearchGui: This class extends the eclipse.jface.dialogs.Dialog class and is almost identical to the ModelSearchGui class. The main difference is that this class takes in the current set of search results, and invokes the appropriate database service methods to search for the user entered criteria within the current search result set. The new search result set is then made available to the code that instantiated this class.

- ModelSearchResultsGui: This class extends the jface.dialogs.Dialog class and is responsible for generating the user interface window that presents the search results to the user. This class contain a number of features that help the user select 
the model that they desire; the following describes how this class provides these features:

- In Chapter 6 the presentation of the search results in the Search Results Pane and in the Details Pane was described in detail. Here we point to the fact that both of these panes are in fact displaying an $\mathrm{html}$ report document that is generated on the fly by the BIRT tools. This class calls the BIRT utility class methods to generate the reports for the given set of search result objects, and then displays the reports in the appropriate pane. More details about BIRT are presented further on in this appendix.

- Chapter 6 also described that the user is able to view the contents of some of the files of the currently selected item without having to download the item. When a user selects to view the contents of a file the FileSystemUtilities, ZipUtil, and CdxxSftputil classes are used to download the zip file for the selected item (if not already downloaded), unzip the zip file into a temporary directory (if not already unzipped), and finally convert its contents into a text string that can be displayed in a normal text box, and display the text.

- Chapter 6 also described that the MS Word document containing the detailed description for a given model can be opened for viewing by the user without having to download the item in question. When the user selects to view a Word document the FileSystemUtilities, ZipUtil, and 
CdxxSftputil classes are used to download and make available the MS Word document in a temporary directory, and then the classes under package Gui.Download.MsWordWindow are invoked to display the word document in a separate window for the user to read.

- Chapter 6 also described the refine search functionality. This functionality is made available through instantiating the refineSearchGui class described earlier. After the refine search is performed the contents of the Search Results Window is updated with the new result set.

- Finally, having chosen a model to download, the user will click the download button to actually download the selected item. Doing this will instantiate the ModelDownloadGui class which will handle the display and processing of the user's download options.

- ModelDownloadGui: This class extends the jface.dialogs.Dialog class and is responsible for generating the user interface window that gives the user a few options of what exactly they want to download, and where to download the files to. These options were described in detail in previous chapters. When the user has made their choices and selected to initiate a download the FileSystemUtilities, ZipUtil, and CdxxSftputil classes are used to download all the zip files for all the items (specifically all of the child modes of a coupled model being downloaded) and package them into one file to be unzipped into the $\mathrm{CD}++$ Project Folder. In addition, if any Experiments have been selected for download, their zip files are 
also downloaded (note that event and model based experiments are handled separately). Finally the zip wizard classes under the Gui.Download.Wizards package are used to present the user with a zip wizard that enables them to choose exactly which files are added to which $\mathrm{CD}++$ Builder project.

\subsection{2- The Gui.Download.Experiment Package}

This package contains all of the classes that produce and manage the user interface windows responsible for collecting search criteria, displaying search results and downloading Experiments. The following is a brief description of these classes:

- ExperimentSearchGui: This class is very similar to the ModelSearchGui class described in the previous section. This class extends the eclipse.jface.dialogs.Dialog class and is the entry point to the experiment download functionality. It creates the user interface window responsible for collecting the search criteria and the other search options from the user. Just like the ModelSearchGui class, this class calls the appropriate search routines from the Persistence Layer to do the actual search in the database and get the list of search results. The role of this class ends by instantiating the ExpSearchResultsGui class to present the search results to the user.

- The RefineSearchGui class: This class is almost identical to the class with the same name under the Gui.Download.Model Package bescribed in the previous section.

- ExpSerachResultsGui: This class is very similar to the ModelSearchResultsGui class 
described in the previous section with the exception that it deals with experiments instead of models. It provides the same features as the ModelSearchResultsGui.

- ExpDownloadGui: This class extends the jface.dialogs.Dialog class and is responsible for generating the user interface window that gives the user the option of where to download the experiment files to, a new $\mathrm{CD}++$ Builder project or an existing one. When the user selects to initiate a download the FileSystemUtilities, ZipUtil, and CdxxSftpUtil classes are used to download the zip file for the selected experiment. Finally the zip wizard classes under the Gui.Download.Wizards package are used to present the user with a zip wizard that enables them to choose exactly which files are added to the $\mathrm{CD}++$ Builder project.

\subsection{3- The Gui.Download.MsWordWindow Package}

This package contains a couple of classes that are responsible for opening the MS Word detailed description documents in a separate window for the user to look at. The following is a brief description of these classes:

- MsWordViewerBttnLstnr: This class extends an eclipse selectionAdapter class and is used as the implementation of the button press event for the "view details" button found on the search results window. This class makes use of the FileSystemUtilities, ZipUtil, and CdxxSftpUtil class to ensure that the Ms Word document is downloaded into the temporary directory, then it instantiates the 
WordDocDisplayGui class to open the document in a separate window.

- WordDocDisplayGui: This class extends the eclipse ApplicationWindow class. Its main function is to create an OleFrame object and use it to open the passed-in MS Word document in a new window.

\subsection{4- The Gui.Download.Wizards Package}

This package contains a couple of classes that are responsible for presenting the Zip Wizard to the user when models or experiments are downloaded to a $\mathrm{CD}++$ Builder project. The two classes under this package are the RepositoryZipWizard class and the RepositoryZipWizardPage class. Both of these classes are adapted from the standard eclipse workbench wizard for importing resources from a zip file, and are almost identical to it presentation wise. Changes were made to the standard wizard to allow programmatically passing in the name of the file to be unzipped and the default location (CD++ Builder project) in which to unzip the file.

\section{3- Utility Classes}

There are a number of utility classes that are used throughout the CD++ Repository application, and these are all collected in the Repository.Util package. The following is a list of the more important of these utility classes:

- The BirtUtil class: This class contains a number of static methods that are used to generate the search results page for Experiments and Models. The class makes use 
of the BIRT engine (described in the next subsection) and the appropriate BIRT report design files to generate HTML formatted reports that are displayed in the search results page.

- CdxxSftpClient: This class implements an SFTP (secure FTP) client capable of uploading and downloading files to the FTP server specified by the IP address in the configuration.

- FileSystemUtilities: This class contains a number of file system related methods that are used in various places in the application.

- HibernateUtil: This class was fully described earlier in section 6.4.1.

- MaFileParser: This class is used to parse the model definition (.ma) files for each model. By parsing the (.ma) file this class is able to build the tree of models under a coupled model and to determine the input output ports for any model. It has a number of other methods that query the tree of models built by the parser.

- ZipUtil: This class contains methods that are capable of compressing and uncompressing files using the java.util.zip utility.

\section{4- The Business Intelligence Reporting Tool (BIRT)}

BIRT is an Eclipse-based open source reporting system that can be used by Java applications to produce reports from many different kinds of data sources (databases, web 
services, Java objects) [31]. BIRT is made of two main components, first the BIRT Report Designer which is an Eclipse based application that enables users to graphically build a report and specify where the data that populates the report comes from and also specify where this data is displayed in the report. The report designer also enables the user to filter, sort, and do other data transforms in addition to using JavaScript to structure the raw data into information that can be displayed in the report. The second component is the BIRT runtime component which will use the report design file created using the designer to populate a report at runtime.

For the $\mathrm{CD}++$ Repository client application BIRT was used to create four different report design files; these are files with extension (.rptdesign). The first is used to generate the report that displays the list of model search results; the second to generate the report that displays the list of experiment search results; the third is used to generate the report that displays the details of the selected model from the search results; and finally the fourth is used to generate the report that displays the details of the selected experiments from the search results. BIRT is capable of producing reports in many formats including PDF and HTML. For the Purposes of the CD++ Repository the reports are generated as HTML documents which are displayed in the appropriate browser objects in the Search Results window. 